\title{
Fourier coefficients of Klingen's Eisenstein series for principal congruence subgroups of the symplectic group
}

\author{
By Yoshiyuki KitaokA
}

(Received August 5, 1991)

Let us explain the motivation. Let us consider the following problem for positive definite integral matrices $S, T$ of degree $m, n$ respectively: when does the equation $S[X]:={ }^{t} X S X=T$ have a solution in $M_{m, n}(\mathbf{Z})$ ? Suppose $n=1$. The condition $m \geq 2 n+3=5$ yields that the solvability of $S[X]=T$ over $\mathbf{Z}_{p}$ for every prime $p$ implies the solvability over $\mathbf{Z}$ if $T$ is sufficiently large. The similar result for $m=2 n+2=4$ is also valid with some minor restriction on $T$. These can be proved arithmetically, and we have the similar result for $m=2 n+1=3$ by an arithmetic proof with the generalized Riemann hypothesis. Let us look at the above from the view of modular forms. Put $r(S, T):=\sharp\left\{X \in M_{m, n}(\mathbf{Z}) \mid S[X]=T\right\}$, and $\theta_{n}(z, S):=\sum_{g} \exp (\pi i \operatorname{tr} S[g] z)$ where $g$ runs over $M_{m, n}(\mathbf{Z})$ and $z$ is in Siegel's upper half-space $H_{n}:=\left\{z \in M_{n}(\mathbf{C}) \mid z={ }^{t} z, \Im z>0\right\}$. Then $\theta_{n}(z, S)$ is obviously equal to $\sum_{P \geq 0} r(S, P) \exp (\pi i \operatorname{tr} P z)$, which is a modular form. When $n=1$, we decompose it as a sum of Eisenstein series and a cusp form. Thus $r(S, T)$ is a sum of Fourier coefficients of Eisenstein series and a cusp form. If we can show that Fourier coefficients of Eisenstein series are larger than those of a cusp form, we get an asymptotic formula of $r(S, T)$, in particular $r(S, T)>0$. In this way, we can get an asymptotic formula of $r(S, T)$ if $m \geq 2 n+1=3$, but we put minor assumptions on $T$ when $m=2 n+1=3$ and $=2 n+2=4$. Apart from Fourier coefficients of Eisenstein series, estimates of Fourier coefficients of cusp forms which we need is the standard estimate by Hecke when $m \geq 2 n+3=5$, Kloosterman or Eichler's improvement when $m=2 n+2=4$ and a variation of Linnik-Selberg's conjecture on Kloosterman sums when $m=2 n+1=3$. When $n \geq 2, \theta_{n}(z, S)$ is not a sum of Eisenstein series and a cusp form in general. Other kind of modular forms appear. When $m$ is comparatively larger than $n$, they are given by Klingen's Eisenstein series. So, if we get a good estimate of their Fourier coefficients, then we have also an asymptotic formula of $r(S, T)$ and as a by-product $r(S, T)>0$. This strategy works when $m \geq 4 n+4$. (We do not refer to other strategies here.) It does not seem that pushing this way on is wrong. When the weight is sufficiently large, we have 
already a complicated expression for the formula of Fourier coefficients of Klingen's Eisenstein series with level $>1$. From it we can get necessary estimates to have an asymptotic formula of $r(S, T)$ when $m \geq 4 n+4$. But even the well-definedness of Klingen's Eisenstein series of low weight does not seem to have been established at present, and Fourier coefficients do not converge when we consider them for the low weight formally. So, our aim here is to present them in a simpler form so as to discuss the well-definedness.

Through the paper, $r, m, q$ denote positive integers, put $n:=m+r$ and suppose $q \geq 3$.

For a commutative ring $R, M_{k, h}(R)$ is the set of $k \times h$ matrices with entries in $R$ and we put $M_{k}(R):=M_{k, k}(R)$, and $\Lambda_{k}(R):=\left\{\lambda={ }^{t} \lambda \in M_{k}(R)\right\}$. For a matrix $T \in M_{k}(R)$, we write $T^{(k)}$ when we need to clarify the size. For simplicity, we put $M_{k, h}:=M_{k, h}(\mathbf{Z}), M_{k}:=M_{k}(\mathbf{Z})$ and $\Lambda_{k}:=\Lambda_{k}(\mathbf{Z})$ if there is no confusion.

We put $\Lambda_{n}^{\prime}:=\left\{\lambda \in \Lambda_{n}(\mathbf{Q}) \mid 2 \lambda \in \Lambda_{n}\right.$, all diagonals of $\left.\lambda \in \mathbf{Z}\right\}$.

For a subset $G \subset M_{n}(\mathbf{Z}), G(q)$ denotes $\left\{g \in G \mid g \equiv 1_{n} \bmod q\right\}$.

For matrices $A, B, A[B]$ denotes ${ }^{t} B A B$ if it is defined.

$\Gamma_{n}, U_{n}$ stand for $S p(n, \mathbf{Z}), S L_{n}(\mathbf{Z})$ respectively and we put

$$
\Gamma_{n}^{\infty}:=\left\{\left(\begin{array}{cc}
a^{(n)} & b \\
0 & d
\end{array}\right) \in \Gamma_{n}\right\} .
$$

A matrix $M$ is often written as

$$
M=\left(\begin{array}{ll}
M_{1} & M_{2} \\
M_{3} & M_{4}
\end{array}\right)
$$

with specification of the size of one of the $M_{i}$ as in $M=\left(\begin{array}{cc}M_{1}^{(r)} & M_{2} \\ M_{3} & M_{4}\end{array}\right)$. Thus for $M \in \Gamma_{n}$, the usual "c"-part of $M$ is written as $M_{3}^{(n)}$. If there is no confusion, $M_{i}$ is such a submatrix.

We denote Siegel's upper half space $\left\{z \in M_{n}(\mathbf{C}) \mid z={ }^{t} z, \Im z>0\right\}$ by $H_{n}$.

$e(w)$ denotes $\exp (2 \pi i w)$ for a complex number $w$.

For a natural numbers $k, h, k \mid h$ means that $k$ divides $h$, and $k \dagger h$ means that $k$ does not divide $h . k \mid h^{\infty}$ means that $k$ divides $h^{t}$ for a sufficiently large integer $t$.

\section{$\S 1 . \quad$ Preparations}

For $\sigma:=\left(\begin{array}{cc}a^{(m)} & b \\ c & d\end{array}\right) \in \Gamma_{m}$ and $v \in U_{n}$ we put

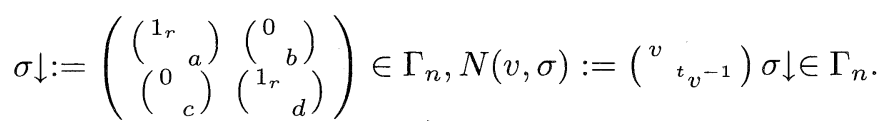

$\Delta_{n, r}$ denotes the subgroup of $\Gamma_{n}$ consisting of $\sigma \in \Gamma_{n}$ such that the left lower $(m, n+r)$ submatrix $\sigma_{3}^{(m, n+r)}:=\left(0,1_{m}\right) \sigma\left(1_{n+r}, 0\right)$ is zero. If so, decomposing $\sigma$ as 
$\sigma=\left(\begin{array}{ll}a & b \\ c & d\end{array}\right)$ at present, $\sigma \in \Delta_{n, r}$ yields $a_{2}=c_{2}=0, c_{3}=d_{3}=0, c_{4}=0$, where we decompose an $n \times n$ matrix $M$ as $M=\left(\begin{array}{cc}M_{1}^{(r)} & M_{2} \\ M_{3} & M_{4}\end{array}\right)$.

$$
P_{n, r} \text { denotes }\left\{\left(\begin{array}{cc}
v_{1}^{(r)} & v_{2} \\
0 & v_{4}
\end{array}\right) \in U_{n}\right\} \text {. }
$$

Lemma 1. Let $u=\left(\begin{array}{cc}u_{1}^{(r)} & u_{2} \\ u_{3} & u_{4}\end{array}\right) \in G L_{n}(\mathbf{C})$ and put $v:={ }^{t} u^{-1}=\left(\begin{array}{cc}v_{1}^{(r)} & v_{2} \\ v_{3} & v_{4}\end{array}\right)$. Then we have $\operatorname{det} u_{4}=\operatorname{det} u \operatorname{det} v_{1}$, and if $\operatorname{det} u_{4} \neq 0$, then we have

$$
\begin{aligned}
& v_{1}={ }^{t}\left(u_{1}-u_{2} u_{4}^{-1} u_{3}\right)^{-1}, \\
& v_{2}=-{ }^{t}\left(u_{1}-u_{2} u_{4}^{-1} u_{3}\right)^{-1 t} u_{3}{ }^{t} u_{4}^{-1}, \\
& v_{3}=-{ }^{t} u_{4}^{-1 t} u_{2}{ }^{t}\left(u_{1}-u_{2} u_{4}^{-1} u_{3}\right)^{-1} \\
& v_{4}={ }^{t} u_{4}^{-1 t} u_{2}{ }^{t}\left(u_{1}-u_{2} u_{4}^{-1} u_{3}\right)^{-1 t} u_{3}{ }^{t} u_{4}^{-1}+{ }^{t} u_{4}^{-1} .
\end{aligned}
$$

Proof. Suppose $\operatorname{det} u_{4} \neq 0$. Then $\left(\begin{array}{cc}1 & -u_{2} u_{4}^{-1} \\ 0 & 1\end{array}\right) u=\left(\begin{array}{cc}u_{1}-u_{2} u_{4}^{-1} u_{3} & 0 \\ u_{3} & u_{4}\end{array}\right)$ and then $\operatorname{det} u=\operatorname{det}\left(u_{1}-u_{2} u_{4}^{-1} u_{3}\right) \operatorname{det} u_{4} \neq 0$. Taking the transpose and the inverse, we have

$$
\left(\begin{array}{cc}
{ }^{t} u_{4}^{-1 t} u_{2} & 1
\end{array}\right) v=\left(\begin{array}{c}
{ }^{t}\left(u_{1}-u_{2} u_{4}^{-1} u_{3}\right)^{-1}-{ }^{t}\left(u_{1}-u_{2} u_{4}^{-1} u_{3}\right)^{-1 t} u_{3}{ }^{t} u_{4}^{-1} \\
0
\end{array}\right) .
$$

Thus $v$ has a desired form, and $\operatorname{det} v_{1}=\operatorname{det}\left(u_{1}-u_{2} u_{4}^{-1} u_{3}\right)^{-1} \operatorname{implies} \operatorname{det} u=$ $\operatorname{det} v_{1}^{-1} \operatorname{det} u_{4}$. If, conversely $\operatorname{det} v_{1} \neq 0$, then $\left(\begin{array}{cc}1 & 0 \\ -v_{3} v_{1}^{-1} & 1\end{array}\right) v=\left(\begin{array}{cc}v_{1} & v_{2} \\ 0 & v_{4}-v_{3} v_{1}^{-1} v_{2}\end{array}\right)$ and so $\left(\begin{array}{cc}1{ }^{t} v_{1}^{-1 t} v_{3} \\ 0 & 1\end{array}\right) u=\left(\begin{array}{c}{ }^{t} v_{1}^{-1} \\ -{ }^{t}\left(v_{4}-v_{3} v_{1}^{-1} v_{2}\right)^{-1 t} v_{2}{ }^{t} v_{1}^{-1}{ }^{t}\left(v_{4}-v_{3} v_{1}^{-1} v_{2}\right)^{-1}\end{array}\right)$. Thus we have

$$
\begin{aligned}
& u_{1}={ }^{t} v_{1}^{-1}+{ }^{t} v_{1}^{-1 t} v_{3}{ }^{t}\left(v_{4}-v_{3} v_{1}^{-1} v_{2}\right)^{-1 t} v_{2}{ }^{t} v_{1}^{-1} \\
& u_{2}=-{ }^{t} v_{1}^{-1 t} v_{3}{ }^{t}\left(v_{4}-v_{3} v_{1}^{-1} v_{2}\right)^{-1} \\
& u_{3}=-{ }^{t}\left(v_{4}-v_{3} v_{1}^{-1} v_{2}\right)^{-1 t} v_{2}{ }^{t} v_{1}^{-1} \\
& u_{4}={ }^{t}\left(v_{4}-v_{3} v_{1}^{-1} v_{2}\right)^{-1}
\end{aligned}
$$

and $\operatorname{det} v_{1} \neq 0$ implies $\operatorname{det} u_{4}=\operatorname{det}^{t}\left(v_{4}-v_{3} v_{1}^{-1} v_{2}\right)^{-1} \neq 0$.

Lemma 2. Let $K=\left(\begin{array}{cc}A^{(n)} & B \\ C & D\end{array}\right) \in \Gamma_{n}$ and suppose $C=\left(\begin{array}{cc}C_{1}^{(r)} & C_{2} \\ 0 & C_{4}\end{array}\right)$. Then the coset $\Delta_{n, r} K$ contains $N(v, \sigma)$ for $v \in U_{n}, \sigma \in \Gamma_{m}$, where $v, \sigma$ can be taken from any fixed representatives of ${ }^{t} P_{n, r} \backslash U_{n}, \Gamma_{m}^{\infty} \backslash \Gamma_{m}$, and we have $\left|\operatorname{det} C_{4}\right|=\mid \operatorname{det} v_{1}^{(r)} \times$ $\operatorname{det} \sigma_{3}^{(m)} \mid$.

Proof. Putting $D:=\left(\begin{array}{cc}D_{1}^{(r)} & D_{2} \\ D_{3} & D_{4}\end{array}\right), K \in \Gamma_{n}$ implies $C^{t} D \in \Lambda_{n}$ and so $C_{4}{ }^{t} D_{4} \in$ $\Lambda_{m}$. By the elementary divisor theorem, we can write $\left(C_{4}, D_{4}\right)=\widetilde{u}_{4}(c, d)$ for 
$\widetilde{u}_{4}, c, d \in M_{m}$ such that $\operatorname{det} \widetilde{u}_{4} \neq 0$ and $(c, d)$ is a primitive matrix. $C_{4}{ }^{t} D_{4} \in \Lambda_{m}$ implies $c^{t} d \in \Lambda_{m}$ and hence there are $a, b \in M_{m}$ such that $\sigma:=\left(\begin{array}{ll}a & b \\ c & d\end{array}\right) \in \Gamma_{m}$. By modifying the decomposition for $\left(C_{4}, D_{4}\right)$ and $a, b$, we may assume that $\sigma$ is one of the fixed representatives of $\Gamma_{m}^{\infty} \backslash \Gamma_{m}$. The last $m$ rows of $K \sigma^{-1} \downarrow$ is $\left(0, C_{4}, D_{3}, D_{4}\right)\left(\begin{array}{ccc}\left(\begin{array}{cc}1_{r} & \\ & { }^{t} d\end{array}\right) & \left(\begin{array}{cc}0 & \\ & -{ }^{t} b\end{array}\right) \\ \left(\begin{array}{cc}0 & \\ & -{ }^{t}{ }_{c}\end{array}\right) & \left(\begin{array}{ll}1_{r} & \\ & { }^{t} a\end{array}\right)\end{array}\right)=\left(0,0, D_{3}, \widetilde{u}_{4}\right)$. Hence $\left(D_{3}, \widetilde{u}_{4}\right)$ is a primitive matrix and there is an extension $\widetilde{u}=\left(\begin{array}{cc}* & * \\ D_{3} & \widetilde{u}_{4}\end{array}\right) \in U_{n}$. Put $\widetilde{u}=w u$ for $w \in P_{n, r}$ and $u$ which is one of the fixed representatives of $P_{n, r} \backslash U_{n}$. Then the last $m$ rows of $K \sigma^{-1} \downarrow\left({ }^{t} u{ }_{u^{-1}}\right)=\left(0,0,0,1_{m}\right)\left({ }^{t} w^{-1}{ }_{w}\right)=(0,0,0, *)$ and hence $K \sigma^{-1} \downarrow$ $\left({ }^{t} u{ }_{u^{-1}}\right) \in \Delta_{n, r}$. Putting $v:={ }^{t} u^{-1}$, we have $\Delta_{n, r} K \ni\left({ }^{v}{ }_{t} v^{-1}\right) \sigma \downarrow=N(v, \sigma)$. $\widetilde{u}=w u$ yields $\operatorname{det} \widetilde{u}_{4}= \pm \operatorname{det} u_{4}^{(m)}$. Since $\operatorname{det} u_{4}=\operatorname{det} v_{1}$ by the previous lemma, we have $\left|\operatorname{det} C_{4}\right|=\left|\operatorname{det} v_{1}\right||\operatorname{det} c|$.

LEMMA 3. We have the following disjoint decomposition:

$$
\Delta_{n}^{r}:=\left\{K \in \Gamma_{n} \mid \operatorname{rank}\left(0,1_{m}\right) K_{3}^{(n)}=m\right\}=\sqcup_{\sigma, v, w} \Delta_{n, r} N(v, \sigma)\left(\begin{array}{ll}
w^{-1} & \\
& { }^{t} w
\end{array}\right)
$$

where $\sigma, v, w$ run over

$$
\left\{\sigma \in \Gamma_{m}^{\infty} \backslash \Gamma_{m} \mid \operatorname{det} \sigma_{3}^{(m)} \neq 0\right\},\left\{v \in{ }^{t} P_{n, r} \backslash U_{n} \mid \operatorname{det} v_{1}^{(r)} \neq 0\right\}, U_{n} / P_{n, r}
$$

respectively. Here $\sigma, v, w$ are taken in any fixed representatives.

Proof. Suppose that $K \in \Delta_{n, r} N(v, \sigma)$ for $v \in U_{n}, \sigma \in \Gamma_{m}$. Then $K_{3}^{(n)}$ is $C v\left(\begin{array}{cc}1_{r} & \\ & \sigma_{1}\end{array}\right)+D^{t} v^{-1}\left(\begin{array}{cc}0 & \\ & \sigma_{3}\end{array}\right)$ for $C=\left(\begin{array}{cc}C_{1}^{(r)} & \\ & 0\end{array}\right), D=\left(\begin{array}{cc}D_{1}^{(r)} & D_{2} \\ 0 & D_{4}\end{array}\right)$. Writing ${ }^{t} v^{-1}=$ $\left(\begin{array}{cc}u_{1}^{(r)} & u_{2} \\ u_{3} & u_{4}\end{array}\right), K_{3}$ is equal to $\left(\begin{array}{cc}* & * \\ 0 & D_{4} u_{4} \sigma_{3}\end{array}\right)$.

If $K \in \Delta_{n}^{r}$, then there is $w \in U_{n}$ such that $K_{3} w=\left(\begin{array}{cc}C_{1}^{(r)} & \stackrel{*}{*} \\ 0 & C_{4}^{(m)}\end{array}\right)$ and $w$ is one of the given representatives. By Lemma 2, there exist $v \in U_{n}, \sigma \in \Gamma_{m}$ such that $K\left({ }^{w}{ }^{{ }^{t}}{ }_{w^{-1}}\right) \in \Delta_{n, r} N(v, \sigma) . \operatorname{rank} C_{4}=m$ implies $\left|\operatorname{det} C_{4}\right|=\left|\operatorname{det} v_{1}\right|\left|\operatorname{det} \sigma_{3}^{(m)}\right| \neq 0$.

Conversely suppose that $K \in \Delta_{n, r} N(v, \sigma)\left(\begin{array}{ll}w^{-1} & \\ & { }^{t} w\end{array}\right)$ for $v, w \in U_{n}, \sigma \in \Gamma_{m}$ with $\operatorname{det} v_{1}^{(r)} \neq 0, \operatorname{det} \sigma_{3}^{(m)} \neq 0$. As above $K_{3}$ is equal to $\left(\begin{array}{cc}* & * \\ 0 & D_{4} u_{4} \sigma_{3}\end{array}\right) w^{-1}$ for $D_{4} \in$ $G L_{m}(\mathbf{Z})$. Since $\operatorname{det}\left(D_{4} u_{4} \sigma_{3}\right)=\operatorname{det}\left(D_{4} v_{1} \sigma_{3}\right) \neq 0, \operatorname{rank}\left(0,1_{m}\right) K_{3}$ is equal to $m$. Thus $K \in \Delta_{n}^{r}$. It remains to show the disjointness. Let $\sigma, v, w$ and $\widetilde{\sigma}, \widetilde{v}, \widetilde{w}$ be one of the given representatives and suppose $\operatorname{det} \sigma_{3}^{(m)} \widetilde{\sigma}_{3}^{(m)} \operatorname{det} v_{1} \widetilde{v}_{1} \neq 0$ and

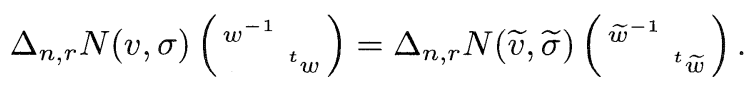


Since

$$
\begin{aligned}
& N(v, \sigma)=\left(\begin{array}{ll}
v & \\
& { }^{t} v^{-1}
\end{array}\right) \sigma \downarrow \\
& =\left(\begin{array}{cc}
v\left(\begin{array}{cc}
1_{r} & \\
& \sigma_{1}
\end{array}\right) & v\left(\begin{array}{cc}
0 & \\
& \sigma_{2}
\end{array}\right) \\
u\left(\begin{array}{cc}
0 & \\
& \sigma_{3}
\end{array}\right) & u\left(\begin{array}{cc}
1_{r} & \\
& \sigma_{4}
\end{array}\right)
\end{array}\right) \quad \text { for } u:={ }^{t} v^{-1} \\
& =\left(\begin{array}{cccc}
* & * & * & * \\
0 & u_{4} \sigma_{3} & u_{3} & u_{4} \sigma_{4}
\end{array}\right) \quad \text { for } u=\left(\begin{array}{cc}
u_{1} & u_{2} \\
u_{3} & u_{4}^{(m)}
\end{array}\right) \text {, }
\end{aligned}
$$

(1) is equivalent to

$$
\left(0, u_{4} \sigma_{3}, u_{3}, u_{4} \sigma_{4}\right)=g\left(0, \widetilde{u}_{4} \widetilde{\sigma}_{3}, \widetilde{u}_{3}, \widetilde{u}_{4} \widetilde{\sigma}_{4}\right)\left(\begin{array}{ll}
\widetilde{w}^{-1} w & \\
& { }^{t}\left(\widetilde{w}^{-1} w\right)
\end{array}\right)
$$

for some $g \in G L_{m}(\mathbf{Z})$, where we put $\widetilde{u}:={ }^{t} \widetilde{v}^{-1}$. Hence $\left(0, u_{4} \sigma_{3}\right)=\left(0, g \widetilde{u}_{4} \widetilde{\sigma}_{3}\right) \widetilde{w}^{-1}$ $\cdot w$ and $\widetilde{w}^{-1} w \in P_{n, r}$ since $\operatorname{det} g \widetilde{u}_{4} \widetilde{\sigma}_{3} \neq 0$. From the assumption $w=\widetilde{w}$ follows. Thus $u_{4}\left(\sigma_{3}, \sigma_{4}\right)=g \widetilde{u}_{4}\left(\widetilde{\sigma}_{3}, \widetilde{\sigma}_{4}\right)$ and hence $\sigma \widetilde{\sigma}^{-1} \in \Gamma_{m}^{\infty}$ and so $\sigma=\widetilde{\sigma}$, which implies $u_{4}\left(\sigma_{3}, \sigma_{4}\right)=g \widetilde{u}_{4}\left(\widetilde{\sigma}_{3}, \widetilde{\sigma}_{4}\right)=g \widetilde{u}_{4}\left(\sigma_{3}, \sigma_{4}\right)$. Hence $u_{4}=g \widetilde{u}_{4}$ and $\left(u_{3}, u_{4}\right)=g\left(\widetilde{u}_{3}, \widetilde{u}_{4}\right)$. This yields $u \in P_{n, r} \widetilde{u}$ and so $v \in{ }^{t} P_{n, r} \widetilde{v}$. This completes the proof.

$$
\begin{gathered}
\text { LEMMA 4. For } v \in U_{n}, \sigma=\left(\begin{array}{ll}
a & b \\
c & d
\end{array}\right) \in \Gamma_{m}, \lambda=\left(\begin{array}{cc}
\lambda_{1}^{(r)} & \lambda_{2} \\
\lambda_{3} & \lambda_{4}
\end{array}\right) \in \Lambda_{n} \text {, we have } \\
N(v, \sigma)\left(\begin{array}{cc}
1_{n} & \lambda \\
0 & 1_{n}
\end{array}\right)=\left(\begin{array}{cc}
1_{n} & x \\
0 & 1_{n}
\end{array}\right) N\left(v\left(\begin{array}{cc}
1_{r}-\lambda_{2}{ }^{t} c \\
0 & 1_{m}
\end{array}\right), \sigma\left(\begin{array}{cc}
1_{m} & \lambda_{4} \\
0 & 1_{m}
\end{array}\right)\right)
\end{gathered}
$$

where

$$
x=v\left(\begin{array}{cc}
\lambda_{1}-\lambda_{2}{ }^{t} a c \lambda_{3} & \lambda_{2}{ }^{t} a \\
a \lambda_{3} & 0
\end{array}\right)^{t} v .
$$

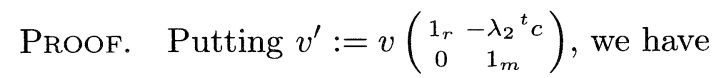

$$
\begin{aligned}
& N(v, \sigma)\left(\begin{array}{cc}
1_{n} & \lambda \\
0 & 1_{n}
\end{array}\right) N\left(v^{\prime}, \sigma\left(\begin{array}{cc}
1_{m} & \lambda_{4} \\
0 & 1_{m}
\end{array}\right)\right)^{-1}
\end{aligned}
$$

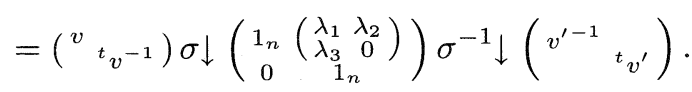

Here $\sigma \downarrow\left(\begin{array}{cc}1_{n} \\ 0\end{array}\left(\begin{array}{cc}\lambda_{1} & \lambda_{2} \\ \lambda_{3} & 0 \\ 1_{n}\end{array}\right)\right) \sigma^{-1} \downarrow$ is equal to

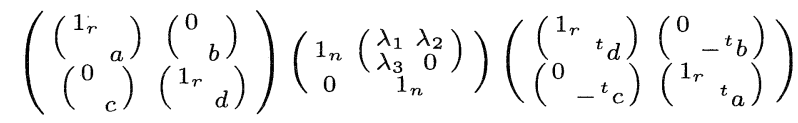

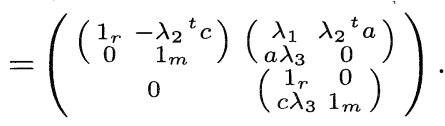


Thus (1) is equal to

$$
\begin{gathered}
\left(\begin{array}{ll}
v & \\
& { }^{t} v^{-1}
\end{array}\right)\left(\begin{array}{cc}
\left(\begin{array}{ccc}
1_{r}-\lambda_{2}{ }^{t} c \\
0 & 1_{m} &
\end{array}\right) & \left(\begin{array}{cc}
\lambda_{1} & \lambda_{2}{ }^{t} a \\
a \lambda_{3} & 0
\end{array}\right) \\
0 & \left(\begin{array}{cc}
1_{r} & 0 \\
c \lambda_{3} & 1_{m}
\end{array}\right)
\end{array}\right)\left(\begin{array}{cc}
\left(\begin{array}{cc}
1_{r} & \lambda_{2}{ }^{t} c \\
0 & 1_{m}
\end{array}\right) \\
\\
\\
=\left(\begin{array}{ll}
v & \\
& { }^{t} v^{-1}
\end{array}\right)\left(\begin{array}{cc}
1_{n} & y \\
0 & 1_{n}
\end{array}\right)\left(\begin{array}{cc}
1_{r} & 0 \\
-c^{t} \lambda_{2} & 1_{m}
\end{array}\right)
\end{array}\right)\left(\begin{array}{ll}
v^{-1} & \\
& { }^{t} v
\end{array}\right)
\end{gathered}
$$

where $y=\left(\begin{array}{cc}\lambda_{1}-\lambda_{2}{ }^{t} a c^{t} \lambda_{2} & \lambda_{2}{ }^{t} a \\ a \lambda_{3} & 0\end{array}\right)=v^{-1} x^{t} v^{-1}$, and hence (1) is equal to $\left(\begin{array}{cc}1_{n} & x \\ 0 & 1_{n}\end{array}\right)$. Thus we have completed the proof.

Lemma 5. For $M \in \Gamma_{n}$, we have

$$
\begin{aligned}
& \left\{K \in \Gamma_{n} \mid \operatorname{rank}\left(0,1_{m}\right) K_{3}^{(n)}=m, K \equiv M \bmod q\right\} \\
& =\sqcup_{w, \lambda, \sigma, v, \eta} \Delta_{n, r}(q) \eta N(v, \sigma)\left(\begin{array}{cc}
1_{n} & \lambda \\
0 & 1_{n}
\end{array}\right)\left(\begin{array}{ll}
w^{-1} & \\
& { }^{t} w
\end{array}\right)
\end{aligned}
$$

where $w, \lambda, \sigma, v$ and $\eta$ run correspondingly as follows:

$$
\begin{aligned}
& w \in U_{n} / P_{n, r}, \\
& \left\{\lambda \in q \Lambda_{n} \mid \lambda_{1}^{(r)} \neq 0\right\}, \\
& \left\{\sigma \in \Gamma_{m}^{\infty} \backslash \Gamma_{m} /\left(\begin{array}{cc}
1_{m} & q \Lambda_{m} \\
0 & 1_{m}
\end{array}\right) \mid \operatorname{det} \sigma_{3}^{(m)} \neq 0\right\}, \\
& \left\{v \in{ }^{t} P_{n, r} \backslash U_{n} /\left(\begin{array}{c}
1_{r} q M_{r, m}{ }^{t} \sigma_{3} \\
0 \\
1_{m}
\end{array}\right) \mid \operatorname{det} v_{1}^{(r)} \neq 0\right\}, \\
& \text { and }\left\{\eta \in \Delta_{n, r}(q) \backslash \Delta_{n, r} \mid \eta N(v, \sigma)\left(w^{-1}{ }^{t} w\right) \equiv M \bmod q\right\} \text {. }
\end{aligned}
$$

Proof. Let $\sigma \in \Gamma_{m}$ with $\operatorname{det} \sigma_{3}^{(m)} \neq 0$ and $v \in U_{n}$ with $\operatorname{det} v_{1}^{(r)} \neq 0$.

We show first that $\Gamma_{m}^{\infty} \sigma=\Gamma_{m}^{\infty} \sigma\left(\begin{array}{cc}1_{m} & \lambda_{4} \\ 0 & 1_{m}\end{array}\right)$ for $\lambda_{4} \in \Lambda_{m}$ implies $\lambda_{4}=0$, and ${ }^{t} P_{n, r} v={ }^{t} P_{n, r} v\left(\begin{array}{cc}1_{r} & \lambda_{2} \\ 0 & 1_{m}\end{array}\right)$ for $\lambda_{2} \in M_{r, m}$ implies $\lambda_{2}=0$. If $\sigma\left(\begin{array}{cc}1_{r} & \lambda_{4} \\ 0 & 1_{m}\end{array}\right) \sigma^{-1} \in \Gamma_{m}^{\infty}$, then $\sigma\left(\begin{array}{cc}1_{r} & \lambda_{4} \\ 0 & 1_{m}\end{array}\right) \sigma^{-1}=\left(\begin{array}{cc}* & * \\ \sigma_{3} \lambda_{4}{ }^{t} \sigma_{3} & *\end{array}\right) \in \Gamma_{m}^{\infty}$ implies $\lambda_{4}=0$ by the assumption $\operatorname{det} \sigma_{3} \neq 0$. If $v\left(\begin{array}{cc}1_{r} & \lambda_{2} \\ 0 & 1_{m}\end{array}\right) v^{-1} \in{ }^{t} P_{n, r}$, then $v\left(\begin{array}{cc}1_{r} & \lambda_{2} \\ 0 & 1_{m}\end{array}\right)=\left(\begin{array}{cc}x_{1}^{(r)} & 0 \\ x_{3} & x_{4}\end{array}\right) v$ for some $\left(\begin{array}{cc}x_{1} & 0 \\ x_{3} & x_{4}\end{array}\right) \in{ }^{t} P_{n, r}$ and it implies $\left(v_{1}, v_{1} \lambda_{2}+v_{2}\right)=\left(x_{1} v_{1}, x_{1} v_{2}\right)$. The assumption $\operatorname{det} v_{1} \neq 0$ yields $x_{1}=1_{r}$ and hence $v_{1} \lambda_{2}+v_{2}=x_{1} v_{2}=v_{2}$ implies $\lambda_{2}=0$.

Now the above facts and Lemma 3 imply

$$
\begin{gathered}
\left\{K \in \Gamma_{n} \mid \operatorname{rank}\left(0,1_{m}\right) K_{3}^{(n)}=m\right\} \\
=\sqcup \Delta_{n, r} N\left(v\left(\begin{array}{cc}
1_{r}-\lambda_{2}{ }^{t} \sigma_{3} \\
0 & 1_{m}
\end{array}\right), \sigma\left(\begin{array}{cc}
1_{m} & \lambda_{4} \\
0 & 1_{m}
\end{array}\right)\right)\left(\begin{array}{ll}
w^{-1} & \\
& { }^{t} w
\end{array}\right),
\end{gathered}
$$


where $w, \lambda_{2}, \lambda_{4}, \sigma, v$ run as follows:

$$
\begin{aligned}
& w \in U_{n} / P_{n, r}, \lambda_{2} \in q M_{r, m}, \lambda_{4} \in q \Lambda_{m}, \\
& \sigma \in \Gamma_{m}^{\infty} \backslash \Gamma_{m} /\left(\begin{array}{cc}
1_{m} & q \Lambda_{m} \\
0 & 1_{m}
\end{array}\right) \quad \text { with } \operatorname{det} \sigma_{3}^{(m)} \neq 0, \\
& v \in{ }^{t} P_{n, r} \backslash U_{n} /\left(\begin{array}{c}
1_{r} \\
0 \\
0 M_{r, m}{ }_{1}^{t}
\end{array} \sigma_{3}{ }^{t}\right) \quad \text { with } \operatorname{det} v_{1}^{(r)} \neq 0 .
\end{aligned}
$$

From Lemma 4 follows

$$
\Delta_{n, r} N(v, \sigma)\left(\begin{array}{cc}
1_{n} & \lambda \\
0 & 1_{n}
\end{array}\right)=\Delta_{n, r} N\left(v\left(\begin{array}{c}
1_{r}-\lambda_{2}^{(r, m) t} \sigma_{3}{ }^{(m)} \\
0 \\
1_{m}
\end{array}\right), \sigma\left(\begin{array}{cc}
1_{m} & \lambda_{4} \\
0 & 1_{m}
\end{array}\right)\right) .
$$

Decomposing $\Delta_{n, r}=\sqcup_{\eta} \Delta_{n, r}(q) \eta\left(\eta \in \Delta_{n, r}(q) \backslash \Delta_{n, r}\right)$, the assertion is clear.

\section{LEMMA 6. We have a disjoint decomposition}

$$
\left\{K \in \Gamma_{n} \mid \operatorname{det} K_{3}^{(n)} \neq 0\right\}=\sqcup_{\sigma, v, M} \Gamma_{n}^{\infty} M \uparrow N(v, \sigma),
$$

where $\sigma, v, M$ run over the following sets, respectively:

$$
\begin{gathered}
\left\{\sigma \in \Gamma_{m}^{\infty} \backslash \Gamma_{m} \mid \operatorname{det} \sigma_{3}^{(m)}>0\right\}, \\
\left\{v \in{ }^{t} P_{n, r} \backslash U_{n} \mid \operatorname{det} v_{1}^{(r)}>0\right\}, \\
\left\{M \in \Gamma_{r}^{\infty} \backslash \Gamma_{r} \mid \operatorname{det} M_{3}^{(r)}>0\right\} .
\end{gathered}
$$

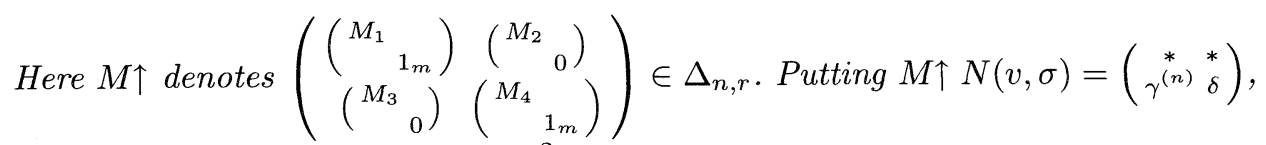
we have $\operatorname{det} \gamma=\operatorname{det} M_{3} \operatorname{det} \sigma_{3} \operatorname{det} v_{1}^{2}$ and

$$
\gamma^{-1} \delta={ }^{t}\left(\gamma^{-1} \delta\right)=\left(\begin{array}{cc}
M_{3}^{-1} M_{4}\left[{ }^{t} v_{1}^{-1}\right]+\sigma_{1} \sigma_{3}^{-1}\left[{ }^{t}\left(v_{1}^{-1} v_{2}\right)\right] & * \\
-\sigma_{3}^{-1 t}\left(v_{1}^{-1} v_{2}\right) & \sigma_{3}^{-1} \sigma_{4}
\end{array}\right)
$$

Proof. For $M \in \Gamma_{r}, v \in U_{n}, \sigma \in \Gamma_{m}$, we have

$$
\begin{aligned}
& M \uparrow N(v, \sigma)
\end{aligned}
$$

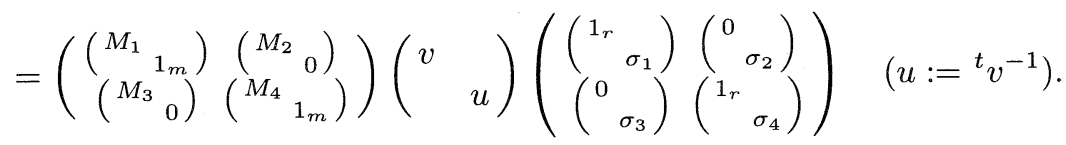

Denoting it by $\left(\begin{array}{cc}* * \\ \gamma & \delta\end{array}\right)$,

$$
\begin{aligned}
& \gamma=\left(\begin{array}{cc}
M_{3} & 0
\end{array}\right) v\left(\begin{array}{cc}
1 & \\
& \sigma_{1}
\end{array}\right)+\left(\begin{array}{ll}
M_{4} & \\
& 1
\end{array}\right) u\left(\begin{array}{cc}
0 & \\
& \sigma_{3}
\end{array}\right)=\left(\begin{array}{cc}
M_{3} v_{1} & M_{3} v_{2} \sigma_{1}+M_{4} u_{2} \sigma_{3} \\
0 & u_{4} \sigma_{3}
\end{array}\right), \\
& \delta=\left(\begin{array}{ll}
M_{3} & 0
\end{array}\right) v\left(\begin{array}{cc}
0 & \\
& \sigma_{2}
\end{array}\right)+\left(\begin{array}{cc}
M_{4} & \\
& 1
\end{array}\right) u\left(\begin{array}{cc}
1 & \\
& \sigma_{4}
\end{array}\right)=\left(\begin{array}{cc}
M_{4} u_{1} & M_{3} v_{2} \sigma_{2}+M_{4} u_{2} \sigma_{4} \\
u_{3} & u_{4} \sigma_{4}
\end{array}\right) .
\end{aligned}
$$


Thus $\operatorname{det} \gamma=\operatorname{det} M_{3} \operatorname{det} \sigma_{3} \operatorname{det} v_{1}^{2} \operatorname{since} \operatorname{det} v_{1}=\operatorname{det} u_{4}$ by Lemma 1.

$$
\gamma^{-1} \delta={ }^{t}\left(\gamma^{-1} \delta\right)=\left(\begin{array}{cc}
M_{3}^{-1} M_{4}\left[{ }^{t} v_{1}^{-1}\right]+\sigma_{1} \sigma_{3}^{-1}\left[{ }^{t}\left(v_{1}^{-1} v_{2}\right)\right] & * \\
-\sigma_{3}^{-1}\left(v_{1}^{-1} v_{2}\right) & \sigma_{3}^{-1} \sigma_{4}
\end{array}\right)
$$

is easy to see, using $v_{1}^{-1} v_{2}=-{ }^{t} u_{3}{ }^{t} u_{4}^{-1}, u_{1}-u_{2} u_{4}^{-1} u_{3}={ }^{t} v_{1}^{-1}$ by Lemma 1 .

Suppose that $\widetilde{M}_{3}^{(n)}$ for $\widetilde{M} \in \Gamma_{n}$ is regular. Then we can take $w \in U_{n}$ so that $\left(\begin{array}{cc}{ }^{t} w^{-1} & \\ & w\end{array}\right) \widetilde{M}=\left(\left(\begin{array}{cc}C_{1}^{(r)} & C_{2} \\ 0 & C_{4}\end{array}\right)^{*}\right)$. From Lemma 2 follows that $\left(\begin{array}{cc}{ }^{t} w^{-1} & \\ & w\end{array}\right) \widetilde{M}$ is equal to $K N(v, \sigma)$ for $K \in \Delta_{n, r}, v \in{ }^{t} P_{n, r} \backslash U_{n}, \sigma \in \Gamma_{m}^{\infty} \backslash \Gamma_{m}$ with $\operatorname{det} C_{4}=$

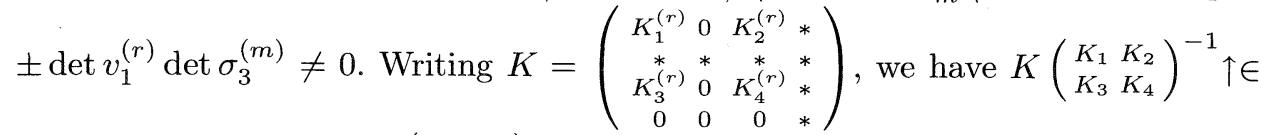
$\Gamma_{n}^{\infty}$. Hence $\Gamma_{n}^{\infty} \widetilde{M}=\Gamma_{n}^{\infty}\left(\begin{array}{ll}K_{1} & K_{2} \\ K_{3} & K_{4}\end{array}\right) \uparrow N(v, \sigma)$ and $\left|\operatorname{det} \widetilde{M}_{3}\right|=\left|\operatorname{det} K_{3} \operatorname{det} \sigma_{3} \operatorname{det} v_{1}^{2}\right|$ from the above. We have to prove the disjointness of the union. Let $\sigma=\left(\begin{array}{ll}a & b \\ c & d\end{array}\right)$, $v=\left(\begin{array}{ll}v_{1} & v_{2} \\ v_{3} & v_{4}\end{array}\right), M=\left(\begin{array}{cc}A & B \\ C & D\end{array}\right)$ and $\widetilde{\sigma}=\left(\begin{array}{cc}\widetilde{a} & \widetilde{b} \\ \widetilde{c} & \widetilde{d}\end{array}\right), \widetilde{v}=\left(\begin{array}{ll}\widetilde{v}_{1} & \widetilde{v}_{2} \\ \widetilde{v}_{3} & \widetilde{v}_{4}\end{array}\right), \widetilde{M}=\left(\begin{array}{cc}\widetilde{A} & \widetilde{B} \\ \widetilde{C} & \widetilde{D}\end{array}\right)$ be representatives in the assertion of Lemma, and suppose $\Gamma_{n}^{\infty} M \uparrow N(v, \sigma)=\Gamma_{n}^{\infty} \widetilde{M} \uparrow N(\widetilde{v}, \widetilde{\sigma})$ with $\operatorname{det} C \widetilde{C} \operatorname{det} v_{1} \widetilde{v}_{1} \operatorname{det} c \tilde{c} \neq 0$. Then for some $U \in G L_{n}(\mathbf{Z})$ we have $\left(\begin{array}{c}* * \\ 0\end{array}\right) M \uparrow$ $N(v, \sigma)=\widetilde{M} \uparrow N(\widetilde{v}, \widetilde{\sigma})$. Comparing the $C$-parts of the both sides, we have

$$
U\left(\begin{array}{cc}
C v_{1} & C v_{2} a+D u_{2} c \\
0 & u_{4} c
\end{array}\right)=\left(\begin{array}{cc}
\widetilde{C} \widetilde{v}_{1} & \widetilde{C} \widetilde{v}_{2} \widetilde{a}+\widetilde{D} \widetilde{u}_{2} \widetilde{c} \\
0 & \widetilde{u}_{4} \widetilde{c}
\end{array}\right)
$$

Hence $U \in P_{n, r}$, and $M \uparrow, \widetilde{M} \uparrow \in \Delta_{n, r}$ implies $\Delta_{n, r} N(v, \sigma)=\Delta_{n, r} N(\widetilde{v}, \widetilde{\sigma})$. By Lemma 3, we have $v=\widetilde{v}, \sigma=\widetilde{\sigma}$. It implies $\Gamma_{n}^{\infty} M \uparrow=\Gamma_{n}^{\infty} \widetilde{M} \uparrow$ and $\left(\widetilde{M} M^{-1}\right) \uparrow \in \Gamma_{n}^{\infty}$. Thus we have $\widetilde{M} M^{-1} \in \Gamma_{r}^{\infty}$ and $\widetilde{M}=M$.

LEMMA 7. $\quad\left(\begin{array}{cc}1_{n} & \Lambda_{n} \\ 0 & 1_{n}\end{array}\right)$ acts faithfully on $\Gamma_{m}^{\infty} \backslash\left\{K \in \Gamma_{n} \mid \operatorname{det} K_{3}^{(n)} \neq 0\right\}$ from the right, and as a complete set of representatives of orbits we can take $M \uparrow N(v, \sigma)$, where $\sigma, v, M$ run, respectively, over

$$
\begin{aligned}
& \left\{\sigma \in \Gamma_{m}^{\infty} \backslash \Gamma_{m} /\left(\begin{array}{cc}
1_{m} & \Lambda_{m} \\
0 & 1_{m}
\end{array}\right) \mid \operatorname{det} \sigma_{3}^{(m)}>0\right\}, \\
& \left\{v \in{ }^{t} P_{n, r} \backslash U_{n} /\left(\begin{array}{cc}
1_{r} & M_{r, m}{ }^{t} \sigma_{3} \\
0 & 1_{m}
\end{array}\right) \mid \operatorname{det} v_{1}^{(r)}>0\right\}, \\
& \left\{M \in \Gamma_{r}^{\infty} \backslash \Gamma_{r} /\left(\begin{array}{cc}
1_{r} & \Lambda_{r}\left[{ }^{t} v_{1}\right] \\
0 & 1_{r}
\end{array}\right) \mid \operatorname{det} M_{3}^{(r)}>0\right\} .
\end{aligned}
$$

Proof. Let $\sigma=\left(\begin{array}{ll}a & b \\ c & d\end{array}\right) \in \Gamma_{m}, v=\left(\begin{array}{ll}v_{1} & v_{2} \\ v_{3} & v_{4}\end{array}\right) \in U_{n}, M=\left(\begin{array}{cc}A & B \\ C & D\end{array}\right) \in \Gamma_{r}$ and $\lambda=\left(\begin{array}{cc}\lambda_{1}^{(r)} & \lambda_{2} \\ \lambda_{3} & \lambda_{4}\end{array}\right) \in \Lambda_{n}$. By Lemma 4 , we have

$$
M \uparrow N(v, \sigma)\left(\begin{array}{cc}
1_{n} & \lambda \\
0 & 1_{n}
\end{array}\right)=M \uparrow\left(\begin{array}{cc}
1_{n} & x \\
0 & 1_{n}
\end{array}\right) N\left(v\left(\begin{array}{cc}
1_{r}-\lambda_{2}{ }^{t} c \\
0 & 1_{m}
\end{array}\right), \sigma\left(\begin{array}{cc}
1_{m} & \lambda_{4} \\
0 & 1_{m}
\end{array}\right)\right)
$$


where $x=v\left(\begin{array}{cc}\lambda_{1}-\lambda_{2}{ }^{t} a c \lambda_{3} & \lambda_{2}{ }^{t} a \\ a \lambda_{3} & 0\end{array}\right)^{t} v$. Putting $x=\left(\begin{array}{cc}x_{1}^{(r)} & x_{2} \\ x_{3} & x_{4}\end{array}\right)$, we have

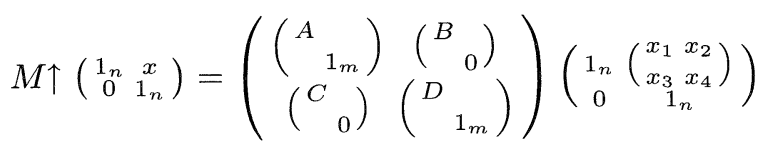

$$
\begin{aligned}
& =\left(\begin{array}{cc}
\left(\begin{array}{c}
A \\
\\
1_{m}
\end{array}\right) & \left(\begin{array}{cc}
A x_{1}+B & A x_{2} \\
x_{3} & x_{4}
\end{array}\right) \\
\left(\begin{array}{ccc}
C & \\
& 0
\end{array}\right) & \left(\begin{array}{cc}
C x_{1}+D & C x_{2} \\
0 & 1_{m}
\end{array}\right)
\end{array}\right) \\
& =\left(\begin{array}{cc}
\left(\begin{array}{cc}
1_{r} & 0 \\
-x_{3}{ }^{t} C & 1_{m}
\end{array}\right)\left(\begin{array}{cc}
0 & A x_{2} \\
0 & \left(\begin{array}{cc}
{ }^{t} & A \\
x_{r} & x_{4}
\end{array}\right) \\
0 & 1_{m}
\end{array}\right)
\end{array}\right)\left(\left(\begin{array}{cc}
A & B \\
C & D
\end{array}\right)\left(\begin{array}{cc}
1_{r} & x_{1} \\
0 & 1_{r}
\end{array}\right)\right) \uparrow .
\end{aligned}
$$

Thus we have

$$
\begin{gathered}
\Gamma_{n}^{\infty} M \uparrow N(v, \sigma)\left(\begin{array}{cc}
1_{n} & \lambda \\
0 & 1_{n}
\end{array}\right) \\
=\Gamma_{n}^{\infty}\left(M\left(\begin{array}{cc}
1_{r} & x_{1} \\
0 & 1_{r}
\end{array}\right)\right) \uparrow N\left(v\left(\begin{array}{cc}
1_{r} & -\lambda_{2}{ }^{t} c \\
0 & 1_{m}
\end{array}\right), \sigma\left(\begin{array}{cc}
1_{m} & \lambda_{4} \\
0 & 1_{m}
\end{array}\right)\right)
\end{gathered}
$$

where $x_{1}:=\left(\begin{array}{cc}\lambda_{1}-\lambda_{2}{ }^{t} a c \lambda_{3} & \lambda_{2}{ }^{t} a \\ a \lambda_{3} & 0\end{array}\right)\left[\begin{array}{c}{ }^{t} v_{1} \\ { }^{t} v_{2}\end{array}\right]=\lambda_{1}\left[{ }^{t} v_{1}\right]-{ }^{t} a c\left[\lambda_{3}{ }^{t} v_{1}\right]+v_{2} a \lambda_{3}{ }^{t} v_{1}+v_{1} \lambda_{2}{ }^{t} a^{t} v_{2}$. By virtue of the previous lemma, our assertion is obvious except for the faithfulness of the action and the completeness of representatives. Let us show these facts. First we remark that, for $k=r$ or $m,\left(\begin{array}{cc}1_{k} & \Lambda_{k} \\ 0 & 1_{k}\end{array}\right)$ acts faithfully from the right on $\Gamma_{k}^{\infty} \backslash\left\{\sigma \in \Gamma_{k} \mid \operatorname{det} \sigma_{3}^{(k)}>0\right\}$ and $\left(\begin{array}{cc}1_{r} & M_{r, m} \\ 0 & 1_{m}\end{array}\right)$ acts also faithfully on ${ }^{t} P_{n, r} \backslash\{v \in$ $\left.U_{n} \mid \operatorname{det} v_{1}^{(r)}>0\right\}$ from the right, as was shown at the begining of the proof of Lemma 5 . Let both $\sigma, v, M$ and $\widetilde{\sigma}, \widetilde{v}, \widetilde{M}$ be as in the assertion of Lemma and assume

$$
\Gamma_{n}^{\infty} M \uparrow N(v, \sigma)\left(\begin{array}{cc}
1_{n} & \lambda \\
0 & 1_{n}
\end{array}\right)=\Gamma_{n}^{\infty} \widetilde{M} \uparrow N(\widetilde{v}, \widetilde{\sigma})
$$

for $\lambda=\left(\begin{array}{cc}\lambda_{1}^{(r)} & \lambda_{2} \\ \lambda_{3} & \lambda_{4}\end{array}\right) \in \Lambda_{n}$. The previous lemma and (1) yield $\Gamma_{m}^{\infty} \widetilde{\sigma}=\Gamma_{m} \sigma\left(\begin{array}{cc}1_{m} & \lambda_{4} \\ 0 & 1_{m}\end{array}\right)$. Since $\widetilde{\sigma}, \sigma$ are representatives of $\Gamma_{m}^{\infty} \backslash \Gamma_{m} /\left(\begin{array}{cc}1_{m} & \Lambda_{m} \\ 0 & 1_{m}\end{array}\right)$, we have $\widetilde{\sigma}=\sigma$ and then $\lambda_{4}=0$ by the above remark. Next ${ }^{t} P_{n, r} v\left(\begin{array}{cc}1_{r}-\lambda_{2}{ }^{t} c \\ 0 & 1_{m}\end{array}\right)={ }^{t} P_{n, r} \widetilde{v}$ holds. By the similar reason we have $v=\widetilde{v}$ and $\lambda_{2}=0$. Then $x_{1}$ is equal to $\lambda_{1}\left[{ }^{t} v_{1}\right]$. Lastly $\Gamma_{r}^{\infty} M\left(\begin{array}{cc}1_{r} & x_{1} \\ 0 & 1_{r}\end{array}\right)=$ $\Gamma_{r}^{\infty} \widetilde{M}$ implies $M=\widetilde{M}$ and $x_{1}=0$, i.e. $\lambda_{1}=0$. Thus we have $M=\widetilde{M}, v=\widetilde{v}, \sigma=\widetilde{\sigma}$ and $\lambda=0$, which complete the proof.

Next we generalize the Möbius transformation. Let us define the Möbius function for finite abelian groups. Let $N$ be a finite abelian group. If $N=\{0\}$, then we put $\mu(N)=0$. If $N$ contains an element whose order is not square-free, then $\mu(N)=0$. Otherwise $N \cong \oplus_{i=1}^{k} \oplus^{r_{i}} \mathbf{Z} / p_{i} \mathbf{Z}$ where $p_{i}$ is a prime number and $p_{i} \neq p_{j}$ 
if $i \neq j$. Then we put $\mu(N)=\prod_{i=1}^{k}(-1)^{r_{i}} p_{i}^{r_{i}\left(r_{i}-1\right) / 2}$. For a natural number $h$, $\mu(\mathbf{Z} / h \mathbf{Z})$ is equal to the authentic Möbius function $\mu(h)$.

LEMMA 8. Let $M$ be a finite abelian group and $S$ the set of subgroups of $M$. Then we have

$$
\sum_{N \in S} \mu(N)=\sum_{N \in S} \mu(M / N)= \begin{cases}1 & \text { if } M=0 \\ 0 & \text { otherwise }\end{cases}
$$

and for functions $\phi, \psi$ on $S$

$$
\psi(K)=\sum_{H \in S, H \supset K} \phi(H) \quad \text { for every } K \in S
$$

if and only if

$$
\phi(H)=\sum_{K \in S, K \supset H} \mu(K / H) \psi(K) \quad \text { for every } H \in S .
$$

This follows from [D].

Now we introduce exponential sums $k$ and $K$. For $T \in \Lambda_{n}(\mathbf{Q}), c, d \in M_{m}$ with $\operatorname{det} c \neq 0, \widetilde{\sigma} \in \Gamma_{m}, v_{1} \in M_{r}(\mathbf{Q}), \widetilde{v}_{2} \in M_{r, m}(\mathbf{Q})$, we define $k, K$ as follows:

Suppose that the following condition ( $\left(\right.$ ) on $T, c, d, \widetilde{\sigma}, v_{1}, \widetilde{v}_{2}$ is satisfied:

$(\natural): T \in \Lambda_{n}^{\prime}, v_{1} \in M_{r}$ with $\operatorname{det} v_{1} \neq 0, T_{1}^{(r)}\left[v_{1}^{-1}\right] \in \Lambda_{r}^{\prime}, \widetilde{v}_{2} \in M_{r, m}$ and there exist $a, b \in M_{m}$ such that $\Gamma_{m} \ni\left(\begin{array}{ll}a & b \\ c & d\end{array}\right) \equiv \widetilde{\sigma} \bmod q$.

Then we put

$$
\begin{aligned}
& k\left(T,(c, d), v_{1} ; \widetilde{\sigma}, \widetilde{v}_{2}\right) \\
& :=\sum_{\substack{v_{2} \in M_{r, m} \bmod q v_{1} M_{r, m} t_{c} \\
v_{2} \equiv \widetilde{v}_{2} \bmod q,\left(v_{1}, v_{2}\right): \text { primitive }}} e\left(\operatorname{tr} T\left(\begin{array}{cc}
a c^{-1}\left[{ }^{t}\left(v_{1}^{-1} v_{2}\right)\right] & \left.\left.-v_{1}^{-1} v_{2}{ }^{t} c^{-1}\right) / q\right), \\
-c^{-1 t}\left(v_{1}^{-1} v_{2}\right) & c^{-1} d
\end{array}\right)\right. \\
& K\left(T,(c, d), v_{1} ; \widetilde{\sigma}, \widetilde{v}_{2}\right) \\
& \quad:=\sum_{\substack{v_{2} \in M_{r, m} \bmod q v_{1} M_{r, m}{ }^{t} c \\
v_{2} \equiv \widetilde{v}_{2} \bmod q}} e\left(\operatorname{tr} T\left(\begin{array}{cc}
a c^{-1}\left[{ }^{t}\left(v_{1}^{-1} v_{2}\right)\right] & -v_{1}^{-1} v_{2}{ }^{t} c^{-1} \\
-c^{-1 t}\left(v_{1}^{-1} v_{2}\right) & c^{-1} d
\end{array}\right) / q\right) .
\end{aligned}
$$

If the condition ( $(\downarrow)$ is not satisfied, then we put $k=K=0$.

Lemma 9. Two exponential sums $k$ and $K$ are well-defined. 
Proof. Suppose that the condition ( $\left(\right.$ a) is satisfied. If $\Gamma_{m} \ni\left(\begin{array}{cc}a^{\prime} & b^{\prime} \\ c & d\end{array}\right) \equiv \sigma \bmod q$, then $\left(\begin{array}{cc}a^{\prime} & b^{\prime} \\ c & d\end{array}\right)=\left(\begin{array}{cc}1_{m} & \lambda \\ 0 & 1_{m}\end{array}\right)\left(\begin{array}{ll}a & b \\ c & d\end{array}\right)$ for $\lambda \in q \Lambda_{m}$. Putting $x=v_{1}^{-1} v_{2}\left(v_{2} \in M_{r, m}\right)$, we want to show

$$
\begin{aligned}
& \operatorname{tr} T\left(\begin{array}{cc}
(a+\lambda c) c^{-1}\left[{ }^{t}\left(x+q y^{t} c\right)\right] & -\left(x+q y t^{c}\right)^{t} c^{-1} \\
-c^{-1 t}\left(x+q y^{t} c\right) & c^{-1} d
\end{array}\right) \\
& \quad \equiv \operatorname{tr} T\left(\begin{array}{cc}
a c^{-1}\left[{ }^{t} x\right] & -x^{t} c^{-1} \\
-c^{-1 t} x & c^{-1} d
\end{array}\right) \bmod q
\end{aligned}
$$

for $y \in M_{r, m}$, which means that the sums $k, K$ are independent of the choice of $v_{2} \bmod q v_{1} M_{r, m}{ }^{t} c$ and the extension $\left(\begin{array}{ll}a & b \\ c & d\end{array}\right) \in \Gamma_{m}$. So the above congruence implies that $k$ and $K$ are well-defined. The left hand side minus the right hand side is equal to

$$
\begin{aligned}
\operatorname{tr} & T_{1}\left((a+\lambda c) c^{-1}\left[{ }^{t}\left(x+q y^{t} c\right)\right]-a c^{-1}\left[{ }^{t} x\right]\right) \bmod q \\
\equiv & \operatorname{tr} T_{1}\left(q x a^{t} y+q y^{t} a^{t} x+q^{2} y^{t} c a^{t} y+\lambda\left[{ }^{t}\left(x+q y^{t} c\right)\right]\right) \bmod q \\
\equiv & q \operatorname{tr} T_{1}\left[v_{1}^{-1}\right]\left(v_{2} a^{t} y^{t} v_{1}+v_{1} y^{t} a^{t} v_{2}+q^{t} c a\left[{ }^{t} y^{t} v_{1}\right]\right) \\
& \quad+\operatorname{tr} T_{1}\left[v_{1}^{-1}\right] \cdot \lambda\left[{ }^{t}\left(v_{2}+q v_{1} y^{t} c\right)\right] \bmod q \\
\equiv & 0 \bmod q
\end{aligned}
$$

since $T_{1}\left[v_{1}^{-1}\right] \in \Lambda_{r}^{\prime}$ and $\lambda \in q \Lambda_{m}$.

It is easy to see that $k, K$ are invariant by replacing $v_{1}, \widetilde{v}_{2}$ by $u v_{1}, u \widetilde{v}_{2}$ for $u \in G L_{r}(\mathbf{Z})$.

LEMma 10. For $T, c, d, v_{1}, \widetilde{\sigma}, \widetilde{v}_{2}$ satisfying the condition ( $($ ), we have

$$
\begin{aligned}
& K\left(T,(c, d), v_{1} ; \widetilde{\sigma}, \widetilde{v}_{2}\right) \\
& =\sum_{g \in M_{r} / G L_{r}(\mathbf{Z})} \sum_{\substack{\widetilde{V}_{2} \in M_{r, m} \bmod q M_{r, m} \\
g \widetilde{V}_{2} \equiv \widetilde{v}_{2} \bmod q}} k\left(T,(c, d), g^{-1} v_{1} ; \widetilde{\sigma}, \widetilde{V}_{2}\right), \\
& k\left(T,(c, d), v_{1} ; \widetilde{\sigma}, \widetilde{v}_{2}\right) \\
& \quad=\sum_{g \in M_{r} / G L_{r}(\mathbf{Z})} \mu\left(\mathbf{Z}^{r} / \mathbf{Z}^{r} g\right) \sum_{\substack{\widetilde{V}_{2} \in M_{r, m} \bmod q M_{r, m} \\
g \widetilde{V}_{2} \equiv \widetilde{v}_{2} \bmod q}} K\left(T,(c, d), g^{-1} v_{1} ; \widetilde{\sigma}, \widetilde{V}_{2}\right) .
\end{aligned}
$$

Here the sums on $g$ are finite. 
Proof. $K\left(T,(c, d), v_{1} ; \widetilde{\sigma}, \widetilde{v}_{2}\right)$ is equal to

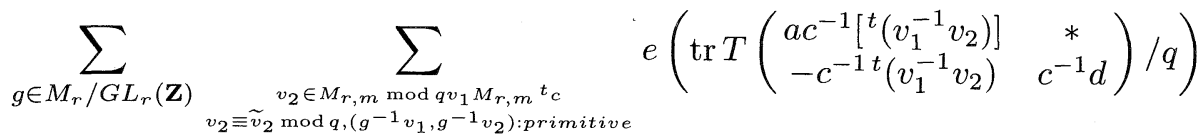

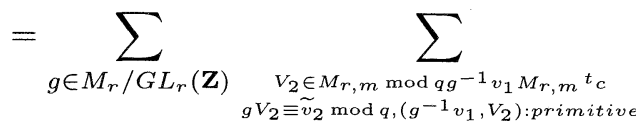

$$
\begin{aligned}
& e\left(\operatorname{tr} T\left(\begin{array}{cc}
a c^{-1}\left[{ }^{t}\left(\left(g^{-1} v_{1}\right)^{-1} V_{2}\right)\right] & * \\
-c^{-1 t}\left(\left(g^{-1} v_{1}\right)^{-1} V_{2}\right) & c^{-1} d
\end{array}\right) / q\right) \\
& =\sum_{g \in M_{r} / G L_{r}(\mathbf{Z})} \sum_{\substack{\widetilde{V}_{2} \in M_{r}, m \bmod q M_{r, m} \\
g \widetilde{V}_{2} \equiv \widetilde{v}_{2} \bmod q}} k\left(T,(c, d), g^{-1} v_{1} ; \widetilde{\sigma}, \widetilde{V}_{2}\right) .
\end{aligned}
$$

Let us identify $G L_{r}(\mathbf{Q}) / G L_{r}(\mathbf{Z})$ with lattices on $\mathbf{Q}^{r}$ by $g \in G L_{r}(\mathbf{Q}) \Leftrightarrow \mathbf{Z}^{r} g^{-1}$. Denote $\mathbf{Z}^{r}$ by $F$. Then for $g \in G L_{r}(\mathbf{Q}), g \in M_{r}$ is equivalent to $F \subset F g^{-1}$. For a lattice $L=F g^{-1} \supset F\left(g \in M_{r}\right)$, we put $\phi(L):=\sum_{\widetilde{V}_{2} \in M_{r, m} \bmod q M_{r, m}} k\left(T,(c, d), g^{-1} v_{1}\right.$; $\left.\widetilde{\sigma}, \widetilde{V}_{2}\right)$. For another choice $g^{\prime}$ for $L=F\left(g^{\prime}\right)^{-1}, g^{\prime}=g u$ for $u \in G L_{r}(\mathbf{Z})$, we have $\sum_{\substack{\widetilde{V}_{2} \in M_{r, m} \bmod q M_{r, m} \\ g^{\prime} \widetilde{V}_{2} \equiv \widetilde{v}_{2} \bmod q}} k\left(T,(c, d),\left(g^{\prime}\right)^{-1} v_{1} ; \widetilde{\sigma}, \widetilde{V}_{2}\right)=\sum_{\substack{\widetilde{V}_{2} \in M_{r, m} \bmod q M_{r, m} \\ g \widetilde{V}_{2} \equiv \widetilde{v}_{2} \bmod q}} k\left(T,(c, d), u^{-1}\right.$ $\left.\cdot g^{-1} v_{1} ; \widetilde{\sigma}, u^{-1} \widetilde{V}_{2}\right)=\sum_{\widetilde{V}_{2} \in M_{r, m} \bmod q M_{r, m}} k\left(T,(c, d), g^{-1} v_{1} ; \widetilde{\sigma}, \widetilde{V}_{2}\right)$, by the remark just $g \widetilde{V}_{2} \equiv \widetilde{v}_{2} \bmod q$

before Lemma 10. Thus $\phi(L)$ is well-defined for $L \supset F$ and $K\left(T,(c, d), v_{1} ; \widetilde{\sigma}, \widetilde{v}_{2}\right)=$ $\sum_{L \supset F} \phi(L)$ by the equation above. Note that $[L: F]=|\operatorname{det} g|>\left|\operatorname{det} v_{1}\right|$ implies $\phi(L)=0$ since $g^{-1} v_{1} \notin M_{r}$. Hence there is a lattice $M \supset F$ such that $L \supset F$ and $\phi(L) \neq 0$ imply $L \subset M$. For a lattice $J=F g_{1}^{-1} \supset F$, we have

$$
\begin{aligned}
& \psi(J):=\sum_{L \supset J} \phi(L) \\
& =\sum_{\substack{g \in M_{r} / G L_{r}(\mathbf{z}) \\
g_{1}^{-1} g \in M_{r}}} \sum_{\substack{\widetilde{V}_{2} \in M_{r, m}, m \bmod q M_{r, m} \\
g \widetilde{V}_{2} \equiv \widetilde{v}_{2} \bmod q}} k\left(T,(c, d), g^{-1} v_{1} ; \widetilde{\sigma}, \widetilde{V}_{2}\right) \\
& =\sum_{G \in M_{r} / G L_{r}(\mathbf{Z})} \sum_{\substack{\widetilde{V}_{2} \in M_{r, m} \bmod q M_{r, m} \\
g_{1} G \widetilde{V}_{2} \equiv \widetilde{v}_{2} \bmod q}} k\left(T,(c, d), G^{-1} g_{1}^{-1} v_{1} ; \widetilde{\sigma}, \widetilde{V}_{2}\right) \\
& =\sum_{G \in M_{r} / G L_{r}(\mathbf{Z})} \sum_{\substack{x \in M_{r, m} \bmod q M_{r, m} \\
g_{1} x \equiv \widetilde{v}_{2} \bmod q}} \sum_{\substack{\widetilde{V}_{2} \in M_{r, m} \bmod q M_{r, m} \\
G \widetilde{V}_{2} \equiv x \bmod q}} k\left(T,(c, d), G^{-1} g_{1}^{-1} v_{1} ; \widetilde{\sigma}, \widetilde{V}_{2}\right) \\
& =\sum_{\substack{x \in M_{r, m} \bmod q M_{r, m} \\
g_{1} \equiv \tilde{v}_{2} \bmod q}} K\left(T,(c, d), g_{1}^{-1} v_{1} ; \widetilde{\sigma}, x\right) .
\end{aligned}
$$


Applying Lemma 8 to $M / F, \phi, \psi$, we have $\phi(F)=\sum_{L \supset F} \mu(L / F) \psi(L)$, which is the second desired equation.

LEMma 11. For $T \in \Lambda_{n}^{\prime}, \widetilde{\sigma} \in \Gamma_{m}, v_{1} \in M_{r}, \widetilde{v}_{2} \in M_{r, m}, \Gamma_{m} \ni\left(\begin{array}{ll}a & b \\ c & d\end{array}\right) \equiv \widetilde{\sigma} \bmod q$ with $\operatorname{det} v_{1} \neq 0$, $\operatorname{det} c \neq 0$, and $u, v \in G L_{m}(\mathbf{Z})$, we have

$$
\begin{aligned}
& K\left(T,(c, d), v_{1} ; \widetilde{\sigma}, \widetilde{v}_{2}\right)=\left|\operatorname{det} v_{1}\right|^{m} K\left(T\left[\left(\begin{array}{ll}
v_{1} & \\
& 1_{m}
\end{array}\right)^{-1}\right],(c, d), 1_{r} ; \widetilde{\sigma}, \widetilde{v}_{2}\right), \\
& K\left(T,\left(u c v, u d^{t} v^{-1}\right), 1_{r} ;\left({ }^{t} u^{-1}{ }_{u}\right) \widetilde{\sigma}\left({ }^{v}{ }_{t} v^{-1}\right), \widetilde{v}_{2}\right) \\
& =K\left(T\left[\left(\begin{array}{ll}
1_{r} & \\
v^{-1}
\end{array}\right)\right],(c, d), 1_{r} ; \widetilde{\sigma}, \widetilde{v}_{2}{ }^{t} u^{-1}\right), \\
& K\left(T\left[\left(\begin{array}{ll}
u & \\
& 1_{m}
\end{array}\right)\right],(c, d), 1_{r} ; \widetilde{\sigma}, \widetilde{v}_{2}\right) \\
& =K\left(T,(c, d), 1_{r} ; \tilde{\sigma}, u \widetilde{v}_{2}\right) \quad \text { for } u \in G L_{r}\left(\mathbf{Z}_{p}\right) .
\end{aligned}
$$

Proof. Writing $v_{2}=x+q y^{t} c$, we can see that $v_{2}$ runs over $M_{r, m} \bmod q v_{1}$ $M_{r, m}{ }^{t} c$ with $v_{2} \equiv \widetilde{v}_{2} \bmod q$ if and only if $x$ runs over $M_{r, m} \bmod q M_{r, m}{ }^{t} c$ with $x \equiv$ $\widetilde{v}_{2} \bmod q$ and $y$ runs over $M_{r, m} \bmod v_{1} M_{r, m}$. Then $a c^{-1}\left[{ }^{t}\left(v_{1}^{-1} v_{2}\right)\right]=a c^{-1}\left[{ }^{t}\left(v_{1}^{-1} x\right)\right]+$ $q v_{1}^{-1}\left(y^{t} a^{t} x+x a^{t} y\right)^{t} v_{1}^{-1}+q^{2 t} c a\left[{ }^{t} y^{t} v_{1}^{-1}\right]$ and $c^{-1 t}\left(v_{1}^{-1} v_{2}\right)=c^{-1 t}\left(v_{1}^{-1} x\right)+q^{t} y^{t} v_{1}^{-1}$ imply $K\left(T,(c, d), v_{1} ; \widetilde{\sigma}, \widetilde{v}_{2}\right)=\sum_{x} e\left(\operatorname{tr} T\left(\begin{array}{cc}a c^{-1}\left[{ }^{t}\left(v_{1}^{-1} x\right)\right] & -v_{1}^{-1} x^{t} c^{-1} \\ -c^{-1 t}\left(v_{1}^{-1} x\right) & c^{-1} d\end{array}\right) / q\right) \times \sum_{y} e(\operatorname{tr} T$ $\left(\begin{array}{c}\left(y^{t} a^{t} x+x a^{t} y+q^{t} c a\left[{ }^{t} y\right]\right)\left[{ }^{t} v_{1}^{-1}\right]-v_{1}^{-1} y \\ -{ }^{t} y^{t} v_{1}^{-1}\end{array}\right)$. If $T_{1}\left[v_{1}^{-1}\right] \notin \Lambda_{r}^{\prime}$, then both sides of (1) are equal to 0 by definition, and so we may assume $T_{1}\left[v_{1}^{-1}\right] \in \Lambda_{r}^{\prime}$. Therefore the partial sum on $y$ turns out to be equal to $\sum_{y} e\left(-2 \operatorname{tr} T_{2}{ }^{t} y^{t} v_{1}^{-1}\right)=\left|\operatorname{det} v_{1}\right|^{m}$ if $2^{t} v_{1}^{-1} T_{2} \in$ $M_{r, m}$ and $=0$ otherwise. Thus we have $K\left(T,(c, d), v_{1} ; \widetilde{\sigma}, \widetilde{v}_{2}\right)=\left|\operatorname{det} v_{1}\right|^{m} K$ $\left(T\left[\left(\begin{array}{cc}v_{1} & \\ & 1_{m}\end{array}\right)^{-1}\right],(c, d), 1_{r} ; \widetilde{\sigma}, \widetilde{v}_{2}\right)$ if $T\left[\left(\begin{array}{ll}v_{1} & \\ & 1_{m}\end{array}\right)^{-1}\right] \in \Lambda_{n}^{\prime}$ and $=0$ otherwise. Since the right hand side of (1) is equal to 0 if $T\left[\left(\begin{array}{cc}v_{1} & \\ & 1_{m}\end{array}\right)^{-1}\right] \notin \Lambda_{n}^{\prime}$, we have proved (1).

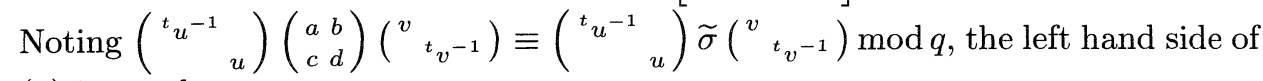
(2) is equal to

$$
\begin{aligned}
& \sum_{\substack{v_{2} \in M_{r, m} \bmod q M_{r, m}{ }^{t}(u c v) \\
v_{2} \equiv \widetilde{v}_{2} \bmod q}} e\left(\operatorname{tr} T\left({ }^{t} u^{-1} a c^{-1} u^{-1}\left[{ }^{t} v_{2}\right] \underset{{ }^{-1} c^{-1} u^{-1 t} v_{2}}{v^{-1} c^{-1} d^{t} v^{-1}}\right) / q\right) \\
& =\sum_{\substack{V_{2} \in M_{r, m} \bmod q M_{r, m} t_{c} \\
V_{2} \equiv v_{2} t^{t} u^{-1} \bmod q}} e\left(\operatorname{tr} T\left(\begin{array}{c}
a c^{-1}\left[{ }^{t} V_{2}\right] \\
-v^{-1} c^{-1 t} V_{2} c^{-1} d\left[{ }^{t} v^{-1}\right]
\end{array}\right) / q\right)
\end{aligned}
$$

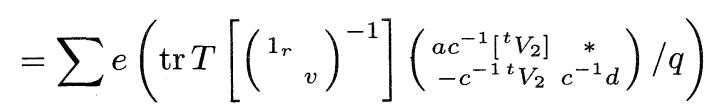

$$
\begin{aligned}
& =K\left(T\left[\left(\begin{array}{cc}
1_{r} \\
& v
\end{array}\right)^{-1}\right],(c, d), 1_{r} ; \widetilde{\sigma}, \widetilde{v}_{2}^{t} u^{-1}\right) \text {. }
\end{aligned}
$$


(3) is easy.

\section{§2. Fourier coefficients of Klingen's Eisenstein series}

From now on, we fix a natural number $k$. Let $f$ be a cusp form of degree $r$, weight $k$ and level $q$. For $M=\left(\begin{array}{ll}a & b \\ c & d\end{array}\right) \in \Gamma_{r}, N \in \Gamma_{n}$, we put

$$
\begin{aligned}
j(M, z) & :=\operatorname{det}(c z+d) \quad \text { for } z \in H_{r}, \\
(f \mid M)(z) & :=j(M, z)^{-k} f(M(z)) \quad \text { for } z \in H_{r}, \\
\left(f \|_{s} N\right)(z) & :=j(N, z)^{-k} f\left(N(z)_{1}\right)\left(\frac{\operatorname{det} \Im N(z)}{\operatorname{det} \Im N(z)_{1}}\right)^{s} \quad \text { for } z \in H_{n},
\end{aligned}
$$

where $N(z)_{1}=N(z)\left[\begin{array}{c}1_{r} \\ 0\end{array}\right]$. By definition, $f(z)$ is holomorphic on $H_{r}$ and satisfies

$$
f \mid M=f \quad \text { for } M \in \Gamma_{r}(q)\left(:=\left\{\sigma \in \Gamma_{r} \mid \sigma \equiv 1_{2 r} \bmod q\right\}\right) .
$$

Note that we consider principal subgroups. It is more convenient to treat cusps impartially. We often use $\|$ instead of $\|_{s}$ for simplicity.

$$
\begin{gathered}
\text { LEMMA 13. For } N=\left(\begin{array}{cccc}
a_{1}^{(r)} & 0 & b_{1}^{(r)} & * \\
* & * & * & * \\
c_{1}^{(r)} & 0 & d_{1}^{(r)} & * \\
0 & 0 & 0 & d_{4}^{(m)}
\end{array}\right) \in \Delta_{n, r} \text { and } M \in \Gamma_{n} \text {, we have } \\
f\left\|N M=\operatorname{det} d_{4}^{-k}\left(f \mid\left(\begin{array}{ll}
a_{1} & b_{1} \\
c_{1} & d_{1}
\end{array}\right)\right)\right\| M .
\end{gathered}
$$

Proof. The proof is straightforward.

$$
\begin{aligned}
j(N M, z) & =j(N, M(z)) j(M, z)=\operatorname{det}\left(\left(\begin{array}{cc}
c_{1} & 0 \\
0 & 0
\end{array}\right) M(z)+\left(\begin{array}{cc}
d_{1} & * \\
0 & d_{4}
\end{array}\right)\right) j(M, z) \\
& =\operatorname{det} d_{4} \operatorname{det}\left(c_{1} M(z)_{1}+d_{1}\right) j(M, z) .
\end{aligned}
$$

$(N M)(z)_{1}=\left(\begin{array}{ll}a_{1} & b_{1} \\ c_{1} & d_{1}\end{array}\right)\left(M(z)_{1}\right)$ is easy. $\Im N M(z)={ }^{t}\left(\left(\begin{array}{cc}c_{1} & \\ & 0\end{array}\right) M(\bar{z})+\left(\begin{array}{cc}d_{1} & * \\ 0 & d_{4}\end{array}\right)\right)^{-1}$ $\Im M(z)\left(\left(\begin{array}{cc}c_{1} & \\ & 0\end{array}\right) M(z)+\left(\begin{array}{cc}d_{1} & * \\ 0 & d_{4}\end{array}\right)\right)^{-1} i m p l i e s \operatorname{det} \Im N M(z)=\operatorname{det} \Im M(z) \mid \operatorname{det}\left(c_{1} M(z)_{1}\right.$ $\left.+d_{1}\right)\left.\right|^{-2}$ and $\operatorname{det} \Im N M(z)_{1}=\operatorname{det} \Im M(z)_{1}\left|\operatorname{det}\left(c_{1} M(z)_{1}+d_{1}\right)\right|^{-2}$. Thus we have

$$
\begin{aligned}
f \| N M(z)= & \operatorname{det} d_{4}^{-k} \operatorname{det}\left(c_{1} M(z)_{1}+d_{1}\right)^{-k} j(M, z)^{-k} \\
& \times f\left(\left(\begin{array}{ll}
a_{1} & b_{1} \\
c_{1} & d_{1}
\end{array}\right)\left(M(z)_{1}\right)\right)\left(\frac{\operatorname{det} \Im M(z)}{\operatorname{det} \Im M(z)_{1}}\right)^{s} \\
= & \operatorname{det} d_{4}^{-k} j(M, z)^{-k}\left(f \mid\left(\begin{array}{ll}
a_{1} & b_{1} \\
c_{1} & d_{1}
\end{array}\right)\right)\left(M(z)_{1}\right)\left(\frac{\operatorname{det} \Im M(z)}{\operatorname{det} \Im M(z)_{1}}\right)^{s},
\end{aligned}
$$


which completes the proof.

Lemma 14. For $M \in \Gamma_{n}, \lambda \in \Lambda_{n}$ and $w \in U_{n}$, we have

$$
\left(f \| M\left(\begin{array}{cc}
1_{n} & \lambda \\
0 & 1_{n}
\end{array}\right)\left(\begin{array}{cc}
w^{-1} & \\
& { }^{t} w
\end{array}\right)\right)(z)=(f \| M)\left(z\left[{ }^{t} w^{-1}\right]+\lambda\right) .
$$

Proof. This follows immediately from $j\left(M\left(\begin{array}{cc}1_{n} & \lambda \\ 0 & 1_{n}\end{array}\right)\left(\begin{array}{ll}w^{-1} & \\ & { }^{t} w\end{array}\right), z\right)=j(M$, $\left.z\left[{ }^{t} w^{-1}\right]+\lambda\right)$.

Lemma 15. For $v \in U_{n}$ with $\operatorname{det} v_{1}^{(r)} \neq 0$ and $\sigma=\left(\begin{array}{ll}a & b \\ c & d\end{array}\right) \in \Gamma_{m}$ with $\operatorname{det}$ $c \neq 0$, we have

$$
\begin{aligned}
& (f \| N(v, \sigma))(z) \\
& =j\left(\sigma, z_{4}\right)^{-k} f\left(z_{1}\left[{ }^{t} v_{1}\right]-\left(z_{4}+c^{-1} d\right)^{-1}\left[z_{3}{ }^{t} v_{1}-c^{-1 t} v_{2}\right]+a c^{-1}\left[{ }^{t} v_{2}\right]\right) \\
& \quad \times\left|\operatorname{det} c \operatorname{det} v_{1}\right|^{-2 s} \operatorname{det}\left(y^{-1}\left[\begin{array}{c}
x_{2}-v_{1}^{-1} v_{2}{ }^{t} c^{-1} \\
x_{4}+c^{-1} d
\end{array}\right]+y_{4}\right)^{-s},
\end{aligned}
$$

where $z=\left(\begin{array}{cc}z_{1}^{(r)} & z_{2} \\ z_{3} & z_{4}\end{array}\right)$ and $x_{i}=\Re z_{i}, y_{i}=\Im z_{i}, y=\Im z$.

Proof. Since $N(v, \sigma)=\left({ }^{v}{ }^{t} v^{-1}\right) \sigma \downarrow$, we have

$$
j(N(v, \sigma), z)=\operatorname{det}\left({ } ^ { t } v ^ { - 1 } \left(\begin{array}{c}
0 \\
\end{array}\right.\right.
$$

and

$$
\begin{aligned}
N(v, \sigma)(z) & =v\left(\left(\begin{array}{cc}
1 & \\
& a
\end{array}\right) z+\left(\begin{array}{ll}
0 & \\
& b
\end{array}\right)\right)\left(\left(\begin{array}{ll}
0 & \\
& c
\end{array}\right) z+\left(\begin{array}{ll}
1 & \\
& d
\end{array}\right)\right)^{-1}{ }^{t} v \\
& =v\left(\begin{array}{cc}
z_{1}-\left(z_{4}+c^{-1} d\right)^{-1}\left[z_{3}\right] & z_{2}\left(z_{4}+c^{-1} d\right)^{-1} c^{-1} \\
{ }^{t} c^{-1}\left(z_{4}+c^{-1} d\right)^{-1} z_{3} & \left(a z_{4}+b\right)\left(c z_{4}+d\right)^{-1}
\end{array}\right){ }^{t} v .
\end{aligned}
$$

Hence $N(v, \sigma)(z)_{1}:=N(v, \sigma)(z)\left[\begin{array}{c}1_{r} \\ 0\end{array}\right]=\left(z_{1}-\left(z_{4}+c^{-1} d\right)^{-1}\left[z_{3}\right]\right)\left[{ }^{t} v_{1}\right]+v_{1} z_{2}\left(z_{4}+\right.$ $\left.c^{-1} d\right)^{-1} c^{-1 t} v_{2}+v_{2}{ }^{t} c^{-1}\left(z_{4}+c^{-1} d\right)^{-1} z_{3}{ }^{t} v_{1}+\left(a z_{4}+b\right)\left(c z_{4}+d\right)^{-1}\left[{ }^{t} v_{2}\right]=z_{1}\left[{ }^{t} v_{1}\right]-$ $\left(z_{4}+c^{-1} d\right)^{-1}\left[z_{3}{ }^{t} v_{1}-c^{-1 t} v_{2}\right]+a c^{-1}\left[{ }^{t} v_{2}\right]$, because of $\left(a z_{4}+b\right)\left(c z_{4}+d\right)^{-1}=a c^{-1}$ $\left(z_{4}+c^{-1} d\right)^{-1}\left[c^{-1}\right]$. It remains to verify

$$
\frac{\operatorname{det} \Im N(v, \sigma)(z)}{\operatorname{det} \Im N(v, \sigma)(z)_{1}}=\operatorname{det} c^{-2} \operatorname{det} v_{1}^{-2} \operatorname{det}\left(y^{-1}\left[\begin{array}{c}
x_{2}-v_{1}^{-1} v_{2}{ }^{t} c^{-1} \\
x_{4}+c^{-1} d
\end{array}\right]+y_{4}\right)^{-1} .
$$

For complex matrices $A, B$, we denote ${ }^{t} \bar{B} A B$ by $A\{B\}$ if it is defined. First let us see for $z=x+i y \in H_{n}$

$$
\operatorname{det} y\left\{z^{-1}\right\} / \operatorname{det} y\left\{z^{-1}\right\}_{1}=\operatorname{det}\left(y^{-1}\left[\begin{array}{l}
x_{2} \\
x_{4}
\end{array}\right]+y_{4}\right)^{-1} .
$$


Putting $Y:=y\left\{z^{-1}\right\}$, we have $Y=\left(x y^{-1} x+y\right)^{-1}$. Decomposing $Y$ as $Y=$ $\left(\begin{array}{cc}Y_{1}^{(r)} & \\ & Y_{4}-Y_{1}^{-1}\left[Y_{2}\right]\end{array}\right)\left[\left(\begin{array}{cc}1_{r} & Y_{1}^{-1} Y_{2} \\ 0 & 1_{m}\end{array}\right)\right]$, we have $\operatorname{det} Y / \operatorname{det} Y_{1}=\operatorname{det}\left(Y_{4}-Y_{1}^{-1}\left[Y_{2}\right]\right)$ and $x y^{-1} x+y=Y^{-1}=\left(\begin{array}{c}* \\ *\left(Y_{4}-Y_{1}^{-1}\left[Y_{2}\right]\right)^{-1}\end{array}\right)$. From these follows (1). Next let us show

$$
\operatorname{det} \Im j(z) / \operatorname{det} \Im j(z)_{1}=\operatorname{det} \Im z / \operatorname{det} \Im z_{1}
$$

for $j:=\left(\begin{array}{cc}0 & -1_{r} \\ 1_{r} & 0\end{array}\right) \uparrow \in \Gamma_{n}$. This follows from $\operatorname{det} \Im j(z) / \operatorname{det} \Im j(z)_{1}=\operatorname{det} y\left\{\left(\left(\begin{array}{cc}1_{r} & \\ & 0\end{array}\right) z\right.\right.$ $\left.\left.+\left(\begin{array}{cc}0 & \\ 1_{m}\end{array}\right)\right)^{-1}\right\} / \operatorname{det} \Im\left(-z_{1}^{-1}\right)=\operatorname{det} y\left|\operatorname{det} z_{1}\right|^{-2} / \operatorname{det} y_{1}\left|\operatorname{det} z_{1}\right|^{-2}=\operatorname{det} y / \operatorname{det} y_{1}$. We apply this to $N(v, \sigma) z$ instead of $z$. To do so, we calculate $\Im j N(v, \sigma)(z)$. Since

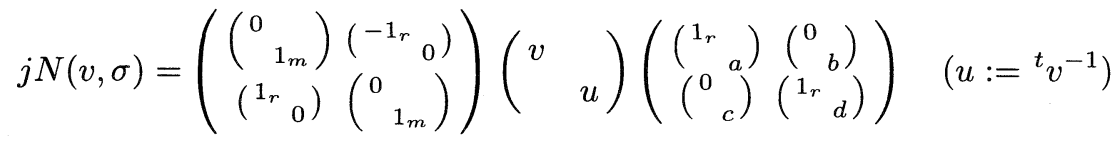

$$
\begin{aligned}
& =\left(\begin{array}{cccc}
0 & -u_{2} c & -u_{1} & -u_{2} d \\
v_{3} & v_{4} a & 0 & v_{4} b \\
v_{1} & v_{2} a & 0 & v_{2} b \\
0 & u_{4} c & u_{3} & u_{4} d
\end{array}\right) \\
& \Im j N(v, \sigma)(z)=y\left\{\left(\left(\begin{array}{cc}
v_{1} & v_{2} a \\
0 & u_{4} c
\end{array}\right) z+\left(\begin{array}{cc}
0 & v_{2} b \\
u_{3} & u_{4} d
\end{array}\right)\right)^{-1}\right\}
\end{aligned}
$$

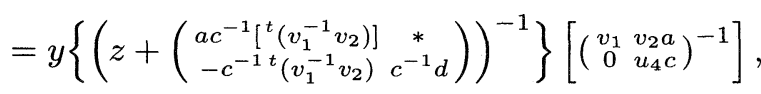

because of $u_{4}^{-1} u_{3}=-{ }^{t}\left(v_{1}^{-1} v_{2}\right)$. Thus we have

$$
\begin{aligned}
& \operatorname{det} \Im N(v, \sigma)(z) / \operatorname{det} \Im N(v, \sigma)(z)_{1} \\
& =\operatorname{det} \Im j N(v, \sigma)(z) / \operatorname{det} \Im j N(v, \sigma)(z)_{1} \\
& =\frac{\operatorname{det} y\left\{\left(z+\left(\begin{array}{cc}
a c^{-1}\left[{ }^{t}\left(v_{1}^{-1} v_{2}\right)\right] & * \\
-c^{-1 t}\left(v_{1}^{-1} v_{2}\right) & c^{-1} d
\end{array}\right)\right)^{-1}\right\} \operatorname{det} v_{1}^{-2} \operatorname{det}\left(u_{4} c\right)^{-2}}{\operatorname{det} y\left\{\left(z+\left(\begin{array}{cc}
a c^{-1}\left[^{t}\left(v_{1}^{-1} v_{2}\right)\right] & * \\
-c^{-1}\left(v_{1}^{-1} v_{2}\right) & c^{-1} d
\end{array}\right)\right)^{-1}\right\}_{1} \operatorname{det} v_{1}^{-2}} \\
& =\operatorname{det}\left(y^{-1}\left[\begin{array}{c}
x_{2}-v_{1}^{-1} v_{2}^{t} c^{-1} \\
x_{4}+c^{-1} d
\end{array}\right]+y_{4}\right)^{-1} \operatorname{det} c^{-2} \operatorname{det} v_{1}^{-2} \quad \text { by }(1),
\end{aligned}
$$

which is what we want.

Lemma 16. Matrices with index 1 are upper left $r \times r$ submatrices here. Let $f(z)=\sum_{0<t \in \Lambda_{r}^{\prime}} b(t) e(\operatorname{tr} t z / q)$ be a cusp form of degree $r$, weight $k$ and level $q$, and $\sigma=\left(\begin{array}{ll}a & b \\ c & d\end{array}\right) \in \Gamma_{m}$ with $\operatorname{det} c \neq 0$ and $v \in U_{n}$ with $\operatorname{det} v_{1} \neq 0$. Then we have, for $T \in \Lambda_{n}^{\prime}$ 


$$
\begin{aligned}
& \left.\int_{x \in \Lambda_{n}(\mathbf{R}) / q \Lambda_{n}} \sum_{\substack{\lambda \in q \Lambda_{n} \\
\lambda_{1}=0}}\left(f \|_{s} N(v, \sigma)\left(\begin{array}{cc}
1_{n} & \lambda \\
0 & 1_{n}
\end{array}\right)\right)(z) e(-\operatorname{tr} T x / q)\right) d x \\
& =q^{r(r+1) / 2} H\left(q^{-1} T, y ; s\right) \operatorname{det} c^{-k}\left|\operatorname{det} c \operatorname{det} v_{1}\right|^{-2 s} b\left(T_{1}\left[v_{1}^{-1}\right]\right) \\
& \quad \times e\left(\operatorname{tr} T\left(\begin{array}{cc}
a c^{-1}\left[{ }^{t}\left(v_{1}^{-1} v_{2}\right)\right] & * \\
-c^{-1}\left(v_{1}^{-1} v_{2}\right) & c^{-1} d
\end{array}\right) / q\right)
\end{aligned}
$$

where $x:=\Re z, y:=\Im z$ and we assume $b\left(T_{1}\left[v_{1}^{-1}\right]\right)=0$ unless $0<T_{1}\left[v_{1}^{-1}\right] \in \Lambda_{r}^{\prime}$, and

$$
\begin{aligned}
H(T, y ; s):=e\left(i \operatorname{tr} T_{1} y_{1}\right) & \int_{\substack{\sigma \in \Lambda_{n}(\mathbf{R}) \\
\sigma_{1}=0}} \operatorname{det}\left(\sigma_{4}+i y_{4}\right)^{-k} \operatorname{det}\left(y^{-1}\left[\begin{array}{c}
\sigma_{2} \\
\sigma_{4}
\end{array}\right]+y_{4}\right)^{-s} \\
& \times e\left(-\operatorname{tr} T_{1} \cdot z_{4}^{-1}\left[z_{3}\right]-\operatorname{tr}\left(\begin{array}{cc}
0 & \sigma_{2} \\
\sigma_{3} & \sigma_{4}
\end{array}\right) T\right) d \sigma_{2} d \sigma_{4}
\end{aligned}
$$

where we put $z_{j}=\sigma_{j}+i y_{j}$ and $d \sigma_{2}=\prod_{i, j} d a_{i j}$ for $\sigma_{2}=\left(a_{i j}\right)$ and $d \sigma_{4}=\prod_{i \leq j} d b_{i j}$ for $\sigma_{4}=\left(b_{i j}\right)$.

Proof. Since $\left(f \| N(v, \sigma)\left(\begin{array}{cc}1_{n} & \lambda \\ 0 & 1_{n}\end{array}\right)\right)(z)=(f \| N(v, \sigma))(z+\lambda)$ by Lemma 14 , the integral in question is equal to

$$
\begin{aligned}
& \int_{x \in \Lambda_{n}(\mathbf{R}) / q \Lambda_{n}} \sum_{\substack{\lambda \in q \Lambda_{n} \\
\lambda_{1}=0}}(f \| N(v, \sigma))(z+\lambda) e(-\operatorname{tr} T x / q) d x \\
& =\int_{\substack{x_{1} \in \Lambda_{r}(\mathbf{R}) / q \Lambda_{r}, x_{2} \in M_{r, m}(\mathbf{R}) \\
x_{4} \in \Lambda_{m}(\mathbf{R})}}(f \| N(v, \sigma))(x+i y) e(-\operatorname{tr} T x / q) d x \\
& =\int_{\substack{x_{1} \in \Lambda_{r}(\mathbf{R}) / q \Lambda_{r}, x_{2} \in M_{r, m}(\mathbf{R}) \\
x_{4} \in \Lambda_{m}(\mathbf{R})}} \operatorname{det}\left(c z_{4}+d\right)^{-k}\left|\operatorname{det} c \operatorname{det} v_{1}\right|^{-2 s} \operatorname{det}\left(y^{-1}\left[\begin{array}{c}
\left.x_{2}-v_{1}^{-1} v^{2}{ }^{t} c^{-1}\right] \\
x_{4}+c^{-1} d
\end{array}\right]+y_{4}\right)^{-s} \\
& \quad \times \sum_{0<t \in \Lambda_{r}^{\prime}} b(t) e\left(\operatorname{tr} t\left(z_{1}\left[^{t} v_{1}\right]-\left(z_{4}+c^{-1} d\right)^{-1}\left[z_{3}{ }^{t} v_{1}-c^{-1 t} v_{2}\right]+a c^{-1}\left[{ }^{t} v_{2}\right]\right) / q\right) \\
& \quad \times e(-\operatorname{tr} T x / q) d x, \\
& =\operatorname{det} c^{-k}\left|\operatorname{det} c \operatorname{det} v_{1}\right|^{-2 s} \sum_{0<t \in \Lambda_{r}^{\prime}} b(t) e\left(i \operatorname{tr} t\left[v_{1}\right] y_{1} / q+\operatorname{tr} t \cdot a c^{-1}\left[{ }^{t} v_{2}\right] / q\right) \\
& \quad \times \int_{x_{1} \in \Lambda_{r}(\mathbf{R}) / q \Lambda_{r}} e\left(\operatorname{tr}\left(t\left[v_{1}\right]-T_{1}\right) x_{1} / q\right) d x_{1}
\end{aligned}
$$




$$
\begin{aligned}
& \times \int_{\substack{x_{2} \in M_{r, m}(\mathbf{R}) \\
x_{4} \in \Lambda m(\mathbf{R})}} \operatorname{det}\left(z_{4}+c^{-1} d\right)^{-k} \operatorname{det}\left(y^{-1}\left[\begin{array}{c}
x_{2}-v_{1}^{-1} v_{2}{ }^{t} c^{-1} \\
x_{4}+c^{-1} d
\end{array}\right]+y_{4}\right)^{-s} \\
& \times e\left(\operatorname{tr} t\left(-\left(z_{4}+c^{-1} d\right)^{-1}\left[z_{3}{ }^{t} v_{1}-c^{-1 t} v_{2}\right]\right) / q-\operatorname{tr}\left(\begin{array}{cc}
0 & T_{2} \\
T_{3} & T_{4}
\end{array}\right)\left(\begin{array}{cc}
0 & x_{2} \\
{ }^{t} x_{2} & x_{4}
\end{array}\right) / q\right) d x_{2} d x_{4} \\
= & q^{r(r+1) / 2} \operatorname{det} c^{-k}\left|\operatorname{det} c \operatorname{det} v_{1}\right|^{-2 s} b\left(T_{1}\left[v_{1}^{-1}\right]\right) e\left(\operatorname{tr} T_{1} \cdot a c^{-1}\left[{ }^{t}\left(v_{1}^{-1} v_{2}\right)\right] / q\right) \\
& \times e\left(\operatorname{tr}\left(\begin{array}{cc}
0 & T_{2} \\
T_{3} & T_{4}
\end{array}\right)\left(\begin{array}{cc}
0 & -v_{1}^{-1} v_{2}{ }^{t} c^{-1} \\
-c^{-1 t}\left(v_{1}^{-1} v_{2}\right) & c^{-1} d
\end{array}\right) / q\right) H\left(q^{-1} T, y ; s\right) .
\end{aligned}
$$

REMARK. Although we did not refer to them explicitly, the convergence and the commutativity of sums and integrals are justified if $\Re s$ is sufficiently large. $H(T, y ; s)$ is absolutely convergent when $\Re s>(n-k) / 2$, and $H(T, y ; s)=$ $O\left(\left(\operatorname{det} T_{1}^{(r)}\right)^{-m / 2}\left(\operatorname{det} y_{4}^{(m)}\right)^{(n+1) / 2-k-s} \exp \left(-2 \pi \operatorname{tr} T_{1}\left(y_{1}-y_{4}^{-1}\left[{ }^{t} y_{2}\right]\right)\right)\right)$ if $\Re s \quad>$ $(n-k) / 2$ and $\Re s \geq 0$. For $w=\left(\begin{array}{cc}w_{1}^{(r)} & w_{2} \\ 0 & w_{4}\end{array}\right) \in G L_{n}(\mathbf{R})$,

$$
H\left(T[w], y\left[{ }^{t} w^{-1}\right] ; s\right)=\left|\operatorname{det} w_{1}\right|^{-m}\left|\operatorname{det} w_{4}\right|^{2 k-n-1+2 s} H(T, y ; s) .
$$

In particular, $H\left(T, T^{-1} ; s\right)=\operatorname{det} T^{-(n+1) / 2+k+s} \operatorname{det} T_{1}^{(r+1) / 2-k-s} H\left(1_{n}, 1_{n} ; s\right)$. It is not difficult to see

$$
\begin{aligned}
H(T, y ; 0)= & 2^{-(n-1) m / 2} i^{-m k}(2 \pi)^{m(k-r / 2)} \pi^{-m(m-1) / 4} \prod_{j=0}^{m-1} \Gamma(k-(r+j) / 2)^{-1} \\
& \times \exp (-2 \pi \operatorname{tr} T y) \begin{cases}\operatorname{det} T_{1}^{(r+1) / 2-k} \operatorname{det} T^{k-(n+1) / 2} & \text { if } T>0, \\
0 & \text { otherwise }\end{cases}
\end{aligned}
$$

when $k>n$.

Lemma 17. Let $f(z)$ be the cusp form in the previous lemma, and for $\sigma=$ $\left(\begin{array}{ll}a & b \\ c & d\end{array}\right) \in \Gamma_{m}$ with $\operatorname{det} c \neq 0$ and for $\widetilde{v}_{1} \in M_{r}, \widetilde{v}_{2} \in M_{r, m}$, we have

$$
\begin{aligned}
& q^{-r(r+1) / 2} \int_{x \in \Lambda_{n}(\mathbf{R}) / q \Lambda_{n}} \sum_{\lambda, v_{1}, v_{2}:(C)}\left(f \| N(v, \sigma)\left(\begin{array}{cc}
1_{n} & \lambda \\
0 & 1_{n}
\end{array}\right)\right)(z) e(-\operatorname{tr} T x / q) d x \\
& =\left[G L_{r}(\mathbf{Z}): U_{r}(q)\right]^{-1} H\left(q^{-1} T, y ; s\right) \operatorname{det} c^{-k}|\operatorname{det} c|^{-2 s} \\
& \quad \times \sum_{v_{1} \in U_{r}(q) \backslash\left(M_{r} \cap G L_{r}(\mathbf{Q})\right)}\left|\operatorname{det} v_{1}\right|^{m-2 s} \\
& \times \sum_{\substack{g \in U_{r}(q) \backslash\left(M_{r} \cap G L_{r}(\mathbf{Q})\right) \\
g v_{1} \equiv \tilde{v}_{1} \bmod q}} \mu\left(\mathbf{Z}^{r} / \mathbf{Z}^{r} g\right)|\operatorname{det} g|^{-2 s} b\left(T_{1}\left[v_{1}^{-1} g^{-1}\right]\right) \\
& \times \sum_{\substack{\widetilde{V}_{2} \in M_{r, m \bmod q M_{r, m}} \\
g \widetilde{V}_{2} \equiv \widetilde{v}_{2} \bmod q}} K\left(T\left[\left(v_{1}^{-1} 1_{m}\right)\right],(c, d), 1_{r} ; \widetilde{\sigma}, \widetilde{V}_{2}\right),
\end{aligned}
$$


where the condition $(\mathrm{C})$ on $\lambda, v_{1}, v_{2}$ is

(C): $\lambda$ runs over $q \Lambda_{n}$ with $\lambda_{1}^{(r)}=0, v_{1}$ runs over $U_{r}(q) \backslash M_{r}$ with $\operatorname{det} v_{1} \neq 0$ and $v_{1} \equiv \widetilde{v}_{1} \bmod q$, and $v_{2}$ runs over $M_{r, m} \bmod q v_{1} M_{r, m}{ }^{t} c$ with $\left(v_{1}, v_{2}\right)$ being primitive and $v_{2} \equiv \widetilde{v}_{2} \bmod q$, and for $v_{1}, v_{2}$ above $v=\left(\begin{array}{cc}v_{1} & v_{2} \\ * & *\end{array}\right)$ is any extension to an element in $U_{n}$.

Proof. Denoting the left hand side of the equation in Lemma by $I$, the previous lemma yields

$$
\begin{aligned}
I= & H\left(q^{-1} T, y ; s\right) \operatorname{det} c^{-k}|\operatorname{det} c|^{-2 s} \\
& \times \sum_{v_{1}}\left|\operatorname{det} v_{1}\right|^{-2 s} b\left(T_{1}\left[v_{1}^{-1}\right]\right) k\left(T,(c, d), v_{1} ; \widetilde{\sigma}, \widetilde{v}_{2}\right),
\end{aligned}
$$

where $v_{1}$ runs over the set in (C). By Lemma 10 , the partial sum on $v_{1}$ is equal to

$$
\begin{aligned}
& \sum_{v_{1}}\left|\operatorname{det} v_{1}\right|^{-2 s} b\left(T_{1}\left[v_{1}^{-1}\right]\right) \sum_{g \in M_{r} / G L_{r}(\mathbf{Z})} \mu\left(\mathbf{Z}^{r} / \mathbf{Z}^{r} g\right) \\
& \times \sum_{\substack{\widetilde{v}_{2} \in M_{r}, m \bmod q M_{r, m} \\
g \widetilde{V}_{2} \equiv \widetilde{v}_{2} \bmod q}} K\left(T,(c, d), g^{-1} v_{1} ; \widetilde{\sigma}, \widetilde{V}_{2}\right) \\
& =\left[G L_{r}(\mathbf{Z}): U_{r}(q)\right]^{-1} \sum_{v_{1}}\left|\operatorname{det} v_{1}\right|^{-2 s} b\left(T_{1}\left[v_{1}^{-1}\right]\right) \sum_{g \in M_{r} / U_{r}(q)} \mu\left(\mathbf{Z}^{r} / \mathbf{Z}^{r} g\right) \\
& \times \sum_{\substack{\widetilde{V}_{2} \in M_{r, m} \bmod q M_{r, m} \\
g \widetilde{V}_{2} \equiv \widetilde{v}_{2} \bmod q, g^{-1} v_{1} \in M_{r}}}\left|\operatorname{det} g^{-1} v_{1}\right|^{m} K\left(T\left[\left(\begin{array}{cc}
v_{1}^{-1} g & \\
& 1_{m}
\end{array}\right)\right],(c, d), 1_{r} ; \widetilde{\sigma}, \widetilde{V}_{2}\right) \\
& =\left[G L_{r}(\mathbf{Z}): U_{r}(q)\right]^{-1} \sum_{v_{1}}\left|\operatorname{det} v_{1}\right|^{m-2 s} b\left(T_{1}\left[v_{1}^{-1}\right]\right) \sum_{\substack{g \in M_{r} / U_{r}(q) \\
g^{-1} v_{1} \in M_{r}}} \mu\left(\mathbf{Z}^{r} / \mathbf{Z}^{r} g\right) \\
& \times|\operatorname{det} g|^{-m} \sum_{\substack{\widetilde{V}_{2} \in M_{r, m \bmod q M_{r, m}} \\
g \widetilde{V}_{2} \equiv \widetilde{v}_{2} \bmod q}} K\left(T\left[\left(\begin{array}{ll}
v_{1}^{-1} g & \\
& 1_{m}
\end{array}\right)\right],(c, d), 1_{r} ; \widetilde{\sigma}, \widetilde{V}_{2}\right),
\end{aligned}
$$

where $v_{1}$ runs over the set in $(\mathrm{C})$. It is easy to see that $\left(\begin{array}{c}g \\ v_{1}\end{array}\right)$ runs over

$$
U_{r}(q) \backslash S_{1} /\left(\begin{array}{ll}
U_{r}(q) & \\
& 1_{r}
\end{array}\right)
$$

$$
\text { for } S_{1}:=\left\{\left(\begin{array}{c}
g \\
v
\end{array}\right) \mid g, v \in M_{r} \cap G L_{r}(\mathbf{Q}), g^{-1} v \in M_{r}, v \equiv \widetilde{v}_{1} \bmod q\right\} \text {. }
$$


Let us consider the mapping $\phi:\left(\begin{array}{l}g \\ v\end{array}\right) \mapsto\left({ }_{t}\left(g^{-1} v\right)\right)$ from this set to

$$
\begin{gathered}
(\mathfrak{\natural}):\left(\begin{array}{ll}
U_{r}(q) & \\
& 1_{r}
\end{array}\right) \backslash S_{2} /\left\{\left(\begin{array}{ll}
{ }^{w}{ }^{t} w^{-1}
\end{array}\right) \mid w \in U_{r}(q)\right\} \\
\text { for } S_{2}:=\left\{\left(\begin{array}{l}
G \\
V
\end{array}\right) \mid G, V \in M_{r} \cap G L_{r}(\mathbf{Q}), G^{t} V \equiv \widetilde{v}_{1} \bmod q\right\} .
\end{gathered}
$$

For $u, v \in U_{r}(q), \phi\left(\begin{array}{c}u g w \\ u v\end{array}\right)=\left(\begin{array}{cc}u & \\ & 1_{r}\end{array}\right)\left(\begin{array}{cc}g & \\ { }^{t} v_{v^{-1} t} g^{-1}\end{array}\right)\left(\begin{array}{cc}w & \\ & { }^{t} w^{-1}\end{array}\right)$ yields that the mapping $\phi$ is well-defined. If $\phi\left(\begin{array}{c}g_{1} \\ v_{1}\end{array}\right)=\left(\begin{array}{c}u \\ { }_{1}\end{array}\right) \phi\left(\begin{array}{c}g_{2} \\ v_{2}\end{array}\right)\left(\begin{array}{ll}{ }^{w}{ }^{t} w^{-1}\end{array}\right)$ for $u, w \in U_{r}(q)$, then $g_{1}=u g_{2} w$ and ${ }^{t} v_{1}{ }^{t} g_{1}^{-1}={ }^{t} v_{2}{ }^{t} g_{2}^{-1 t} w^{-1}$, which imply $\left(\begin{array}{l}g_{1} \\ v_{1}\end{array}\right)=u\left(\begin{array}{l}g_{2} \\ v_{2}\end{array}\right)\left(\begin{array}{l}w_{1} \\ { }_{1}\end{array}\right)$. Thus $\phi$ is injective. $\phi\left(\begin{array}{c}G \\ G^{t} V\end{array}\right)=\left(\begin{array}{l}G \\ V\end{array}\right)$ means that $\phi$ is surjective and hence $\phi$ is bijective. In the set (Ł), we can choose first $V \in\left(M_{r} \cap G L_{r}(\mathbf{Q})\right) / U_{r}(q)$ and then $G \in U_{r}(q) \backslash\left(M_{r} \cap G L_{r}(\mathbf{Q})\right)$. Thus the above sum turns out to be equal to

$$
\begin{aligned}
& {\left[G L_{r}(\mathbf{Z}): U_{r}(q)\right]^{-1} \sum_{V \in\left(M_{r} \cap G L_{r}(\mathbf{Q})\right) / U_{r}(q)} \sum_{\substack{G \in U_{r}(q) \backslash\left(M_{r} \cap G L_{r}(\mathbf{Q}) \\
G^{t} V \equiv \tilde{v}_{1} m o d q\right.}}|\operatorname{det} G V|^{m-2 s}} \\
& \times b\left(T_{1}\left[{ }^{t} V^{-1} G^{-1}\right]\right) \mu\left(\mathbf{Z}^{r} / \mathbf{Z}^{r} G\right)|\operatorname{det} G|^{-m} \\
& \times \sum_{\substack{\widetilde{V}_{2} \in M_{r, m} \bmod q M_{r, m} \\
G \widetilde{V}_{2} \equiv \widetilde{v}_{2} \bmod q}} K\left(T\left[\left({ }^{t} V^{-1}{ }\right)\right],(c, d), 1_{r} ; \widetilde{\sigma}, \widetilde{V}_{2}\right) .
\end{aligned}
$$

Replacing $V$ by ${ }^{t} v_{1}$ and $G$ by $g$, we get the assertion.

Lemma 18. For the cusp form $f(z)$ in Lemma 16 and $M \in \Gamma_{n}$, we put

$$
\mathcal{F}(T, y ; f, M):=q^{-r(r+1) / 2} \int_{x \in \Lambda_{n}(\mathbf{R}) / q \Lambda_{n}} \sum_{K}(f \| K)(z) e(-\operatorname{tr} T x / q) d x
$$

where $y:=\Im z, x:=\Re z$ and $\sigma$ runs over

$$
\begin{aligned}
& \Delta_{n, r}(q) \backslash\left\{K \in \Gamma_{n} \mid \operatorname{rank}\left(0,1_{m}\right) K_{3}^{(n)}=m, \sigma \equiv M \bmod q\right\} \\
& \text { For } \eta:=\left(\begin{array}{cccc}
a_{1}^{(r)} & 0 & b_{1}^{(r)} & * \\
* & * & * & * \\
c_{1}^{(r)} & 0 & d_{1}^{(r)} & * \\
0 & 0 & 0 & d_{4}
\end{array}\right) \in \Delta_{n, r}, \text { we put } \\
& \\
& \quad \operatorname{det} d_{4}^{-k}\left(f \mid\left(\begin{array}{ll}
a_{1} & b_{1} \\
c_{1} & d_{1}
\end{array}\right)\right)(\tau):=\sum_{t>0} b(t ; \eta) e(\operatorname{tr} t \tau / q) \quad\left(\tau \in H_{r}\right) .
\end{aligned}
$$


Then we have

$$
\begin{aligned}
\mathcal{F} & (T, y ; f, M) \\
= & {\left[\Gamma_{m}^{\infty}: \Gamma_{m}^{\infty}(q)\right]^{-1}\left[P_{n, r}: P_{n, r}(q)\right]^{-2}\left[G L_{r}(\mathbf{Z}): U_{r}(q)\right]^{-1} } \\
& \left.\times \sum_{w} H\left(q^{-1} T[w], y{ }^{t} w^{-1}\right] ; s\right) \\
& \times \sum_{\widetilde{v}, \eta, \widetilde{\sigma}, v_{1}}\left|\operatorname{det} v_{1}\right|^{m-2 s} \sum_{g} \mu\left(\mathbf{Z}^{r} / \mathbf{Z}^{r} g\right)|\operatorname{det} g|^{-2 s} b\left(T[w]_{1}\left[v_{1}^{-1} g^{-1}\right] ; \eta\right) \\
& \times \sum_{\widetilde{V}_{2}} \sum_{(c, d)} \operatorname{det} c^{-k}|\operatorname{det} c|^{-2 s} K\left(T\left[w\left({ }^{v_{1}}{ }_{1 m}\right)^{-1}\right],(c, d), 1_{r} ; \widetilde{\sigma}, \widetilde{V}_{2}\right),
\end{aligned}
$$

where the ranges of sums are

$$
\begin{aligned}
w & \in U_{n} / P_{n, r}(q), \\
\widetilde{v} & =\left(\begin{array}{cc}
\widetilde{v}_{1}^{(r)} & \widetilde{v}_{2} \\
* & *
\end{array}\right) \in U_{n} / U_{n}(q), \\
\eta & \in \Delta_{n, r}(q) \backslash \Delta_{n, r}, \\
\widetilde{\sigma} & =\left(\begin{array}{cc}
\tilde{a} & \widetilde{b} \\
\widetilde{c} & \widetilde{d}
\end{array}\right) \in \Gamma_{m} / \Gamma_{m}(q) \quad \text { with } \quad \eta N(\widetilde{v}, \widetilde{\sigma})\left(\begin{array}{ll}
w^{-1} & \\
& { }^{t} w
\end{array}\right) \equiv M \bmod q, \\
v_{1} & \in U_{r}(q) \backslash\left(M_{r} \cap G L_{r}(\mathbf{Q})\right), \\
g & \in U_{r}(q) \backslash\left(M_{r} \cap G L_{r}(\mathbf{Q})\right) \quad \text { with } g v_{1} \equiv \widetilde{v}_{1} \bmod q, \\
\widetilde{V}_{2} & \in M_{r, m} \bmod q M_{r, m} \quad \text { with } \quad g \widetilde{V}_{2} \equiv \widetilde{v}_{2} \bmod q,
\end{aligned}
$$

and lastly $(c, d)$ runs over

$$
U_{m}(q) \backslash\left\{(c, d) \mid c, d \in M_{m}, \operatorname{det} c \neq 0\right\} /\left(\begin{array}{cc}
1_{m} & q \Lambda_{m} \\
0 & 1_{m}
\end{array}\right) .
$$

Proof. First, let us see that the number of inverse images of the canonical mapping from $\Gamma_{m}^{\infty}(q) \backslash \Gamma_{m} /\left(\begin{array}{cc}1 & q \Lambda_{m} \\ 0 & 1\end{array}\right)$ to $\Gamma_{m}^{\infty} \backslash \Gamma_{m} /\left(\begin{array}{cc}1 & q \Lambda_{m} \\ 0 & 1\end{array}\right)$ is $\left[\Gamma_{m}^{\infty}: \Gamma_{m}^{\infty}(q)\right]$. For $\sigma, \eta \in \Gamma_{m}, \Gamma_{m}^{\infty} \sigma\left(\begin{array}{cc}1 & q \Lambda_{m} \\ 0 & 1\end{array}\right)=\Gamma_{m}^{\infty} \eta\left(\begin{array}{cc}1 & q \Lambda_{m} \\ 0 & 1\end{array}\right)$ implies $\Gamma_{m}^{\infty}(q) \sigma\left(\begin{array}{cc}1 & q \Lambda_{m} \\ 0 & 1\end{array}\right)=\xi \Gamma_{m}^{\infty}(q) \eta$ $\left(\begin{array}{cc}1 & q \Lambda_{m} \\ 0 & 1\end{array}\right)$ for $\xi \in \Gamma_{m}^{\infty}$, and then $\Gamma_{m}^{\infty}(q) \sigma\left(\begin{array}{cc}1 & q \Lambda_{m} \\ 0 & 1\end{array}\right)=\Gamma_{m}^{\infty}(q) \eta\left(\begin{array}{cc}1 & q \Lambda_{m} \\ 0 & 1\end{array}\right)$ holds if and only if $\xi \in \Gamma_{m}^{\infty}(q)$, considering both sides modulo $q$. Thus the number in question is $\left[\Gamma_{m}^{\infty}: \Gamma_{m}^{\infty}(q)\right]$. Next, let us see that the number of inverse images of the canonical mapping from ${ }^{t} P_{n, r}(q) \backslash U_{n} /\left(\begin{array}{cc}1 q M_{r, m}{ }^{t} c \\ 0 & 1\end{array}\right)$ to ${ }^{t} P_{n, r} \backslash U_{n} /\left(\begin{array}{cc}1 q M_{r, m}{ }^{t} c \\ 0 & 1\end{array}\right)$ for $c \in M_{m}$ with $\operatorname{det} c \neq 0$ is $\left[P_{n, r}: P_{n, r}(q)\right]$. For $u, v \in U_{n},{ }^{t} P_{n, r} u\left(\begin{array}{cc}1 & q M_{r, m}{ }^{t} c \\ 0 & 1\end{array}\right)=$ 
${ }^{t} P_{n, r} v\left(\begin{array}{cc}1 & q M_{r, m}{ }^{t} c \\ 0 & 1\end{array}\right)$ implies $u=w v\left(\begin{array}{cc}1 & q y^{t} c \\ 0 & 1\end{array}\right)$ for $w \in{ }^{t} P_{n, r}, y \in M_{r, m}$, and then it is easy to see that ${ }^{t} P_{n, r}(q) u\left(\begin{array}{cc}1 q M_{r, m}{ }^{t} c \\ 0 & 1\end{array}\right)={ }^{t} P_{n, r}(q) v\left(\begin{array}{cc}1 q M_{r, m}{ }^{t} c \\ 0 & 1\end{array}\right)$ if and only if $w \equiv 1_{n} \bmod q$. Thus the number of inverse images is $\left[P_{n, r}: P_{n, r}(q)\right]$. Now applying Lemma 5, we have

$$
\begin{aligned}
& \mathcal{F}(T, y ; f, M) \\
& =q^{-r(r+1) / 2}\left[\Gamma_{m}^{\infty}: \Gamma_{m}^{\infty}(q)\right]^{-1}\left[P_{n, r}: P_{n, r}(q)\right]^{-2} \\
& \quad \times \sum_{w, \lambda, \sigma, v, \eta} \int_{x \in \Lambda_{n}(\mathbf{R}) / q \Lambda_{n}}\left(f \| \eta N(v, \sigma)\left(\begin{array}{ll}
1 & \lambda \\
0 & 1
\end{array}\right)\left(w^{-1}{ }^{t} w\right)\right)(z) e(-\operatorname{tr} T x / q) d x,
\end{aligned}
$$

where sums on $w, \lambda, \sigma, v, \eta$ are taken as follows: $w \in U_{n} / P_{n, r}(q), \lambda \in q \Lambda_{n}$ with $\lambda_{1}^{(r)}=0, \sigma \in \Gamma_{m}^{\infty}(q) \backslash \Gamma_{m} /\left(\begin{array}{cc}1_{m} & q \Lambda_{m} \\ 0 & 1_{m}\end{array}\right)$ with $\operatorname{det} \sigma_{3}^{(m)} \neq 0, v \in{ }^{t} P_{n, r}(q) \backslash U_{n} /$ $\left(\begin{array}{cc}1_{r} & q M_{r, m}{ }^{t} \sigma_{3} \\ 0 & 1_{m}\end{array}\right)$ with $\operatorname{det} v_{1}^{(r)} \neq 0$ and $\eta \in \Delta_{n, r}(q) \backslash \Delta_{n, r}$ with $\eta N(v, \sigma)\left(\begin{array}{cc}w^{-1} & \\ & \\ & { }^{t} w\end{array}\right) \equiv$ $M \bmod q$.

Hence the sum on $w, \lambda, \sigma, v, \eta$ in the above can be replaced by the sum on $w, \widetilde{v}, \eta, \widetilde{\sigma}, \sigma, v, \lambda$ where $w, \widetilde{v}, \eta, \widetilde{\sigma}$ are as in the assertion of Lemma and $\sigma \in \Gamma_{m}^{\infty}(q) \backslash$ $\Gamma_{m} /\left(\begin{array}{cc}1 & q \Lambda_{m} \\ 0 & 1\end{array}\right)$ with $\operatorname{det} \sigma_{3}^{(m)} \neq 0, \sigma \equiv \tilde{\sigma} \bmod q$ and $v$ runs over ${ }^{t} P_{n, r}(q) \backslash U_{n} /$ $\left(\begin{array}{c}1 q M_{r, m}{ }^{t} \sigma_{3} \\ 0 \\ 1\end{array}\right)$ with $\operatorname{det} v_{1}^{(r)} \neq 0, v \equiv \widetilde{v} \bmod q$ and $\lambda$ runs over $q \Lambda_{n}$ with $\lambda_{1}^{(r)}=0$. And the above integral is equal to

$$
\int_{x \in \Lambda_{n}(\mathbf{R}) / q \Lambda_{n}}\left(f \| \eta N(v, \sigma)\left(\begin{array}{ll}
1 & \lambda \\
0 & 1
\end{array}\right)\right)\left(x+i y\left[{ }^{t} w^{-1}\right]\right) e(-\operatorname{tr} T[w] x / q) d x .
$$

Let us define the mapping $v \mapsto\left(1_{r}, 0\right) v$ from $\left\{v=\left(\begin{array}{cc}v_{1}^{(r)} & v_{2} \\ * & *\end{array}\right) \in{ }^{t} P_{n, r}(q) \backslash U_{n} /\right.$ $\left.\left(\begin{array}{c}1 q M_{r, m}{ }^{t} \sigma_{3} \\ 0 \\ 1\end{array}\right) \mid \operatorname{det} v_{1} \neq 0, v \equiv \widetilde{v} \bmod q\right\}$ to $U_{r}(q) \backslash\left\{\left(v_{1}, v_{2}\right) \mid v_{1} \in M_{r}, v_{1} \equiv\right.$ $\widetilde{v}_{1}^{(r)} \bmod q, \operatorname{det} v_{1} \neq 0, v_{2} \in M_{r, m} \bmod q v_{1} M_{r, m}{ }^{t} \sigma_{3}, v_{2} \equiv \widetilde{v}_{2} \bmod q,\left(v_{1}, v_{2}\right):$ primitive $\}$ and verify that this is bijective. The well-definedness is clear.

Suppose that for $v, v^{\prime} \in U_{n},\left(v_{1}^{\prime}, v_{2}^{\prime}\right)=u\left(v_{1}, v_{2}+q v_{1} x^{t} \sigma_{3}\right)$ for $u \in U_{r}(q)$ and $x \in M_{r}$

Then there exist $y \in M_{m, r}$ and $z \in M_{r}$ such that $v^{\prime}=\left(\begin{array}{cc}u & 0 \\ y & z\end{array}\right) v\left(\begin{array}{cc}1_{r} & q x^{t} \sigma_{3} \\ 0 & 1_{m}\end{array}\right)$. $v^{\prime} \equiv v \equiv \widetilde{v} \bmod q$ yields then $\left(\begin{array}{ll}u & 0 \\ y & z\end{array}\right) \equiv 1_{n} \bmod q$ and hence it is in ${ }^{t} P_{n, r}(q)$ and so $v$ and $v^{\prime}$ are in the same double coset. So the mapping is injective. Conversely suppose that $v_{1}, v_{2}$ are given. Since $\left(v_{1}, v_{2}\right)$ is primitive, they can be extended to $v=\left(\begin{array}{cc}v_{1} & v_{2} \\ * & *\end{array}\right) \in U_{n}$, and then $v \widetilde{v}^{-1} \equiv\left(\begin{array}{cc}1_{r} & 0 \\ \gamma, \delta\end{array}\right) \bmod q$ with $\operatorname{det} \delta \equiv 1 \bmod q$ yields that there exists $w=\left(\begin{array}{cc}1_{r} & 0 \\ * & *\end{array}\right) \in{ }^{t} P_{n, r}(q)$ such that $v \widetilde{v}^{-1} \equiv w \bmod q$. Now $\left(v_{1}, v_{2}\right)$ is the image of $w^{-1} v$. Thus the mapping is surjective. Note that the conditions on $v_{1}, v_{2}$ 
are ones given in (C) in Lemma 17, putting $c=\sigma_{3}$. From Lemma 17 follows now that

$$
\begin{aligned}
& \mathcal{F}(T, y ; f, M) \\
& =\left[\Gamma_{m}^{\infty}: \Gamma_{m}^{\infty}(q)\right]^{-1}\left[P_{n, r}: P_{n, r}(q)\right]^{-2}\left[G L_{r}(\mathbf{Z}): U_{r}(q)\right]^{-1} \sum_{w} H\left(q^{-1} T[w], y\left[^{t} w^{-1}\right] ; s\right) \\
& \quad \times \sum_{\widetilde{v}, \eta, \widetilde{\sigma}, \sigma}\left(\operatorname{det} \sigma_{3}^{(m)}\right)^{-k}\left|\operatorname{det} \sigma_{3}\right|^{-2 s} \sum_{v_{1}}\left|\operatorname{det} v_{1}\right|^{m-2 s} \\
& \quad \times \sum_{g} \mu\left(\mathbf{Z}^{r} / \mathbf{Z}^{r} g\right)|\operatorname{det} g|^{-2 s} b\left(T[w]_{1}\left[v_{1}^{-1} g^{-1}\right] ; \eta\right) \\
& \quad \times \sum_{\widetilde{V}_{2}} K\left(T\left[w\left(^{v_{1}}{ }_{1_{m}}\right)^{-1}\right],\left(\sigma_{3}, \sigma_{4}\right), 1_{r} ; \widetilde{\sigma}, \widetilde{V}_{2}\right),
\end{aligned}
$$

where $w, \widetilde{v}, \eta, \widetilde{\sigma}, v_{1}, g, \widetilde{V}_{2}$ run over the sets given in the assertion of Lemma and $\sigma$ runs over the set as above, and $T[w]_{1}=T[w]\left[\begin{array}{c}1_{r} \\ 0\end{array}\right]$ by definition. Finally let us see that $\left\{\sigma=\left(\begin{array}{ll}a & b \\ c & d\end{array}\right) \in \Gamma_{m}^{\infty}(q) \backslash \Gamma_{m} /\left(\begin{array}{cc}1 & q \Lambda_{m} \\ 0 & 1\end{array}\right) \mid \operatorname{det} c \neq 0, \sigma \equiv \widetilde{\sigma} \bmod q\right\}$ and $U_{m}(q) \backslash\left\{(c, d) \mid\left(\begin{array}{c}* * \\ c\end{array}\right) \in \Gamma_{m}, \operatorname{det} c \neq 0,(c, d) \equiv(\widetilde{c}, \widetilde{d}) \bmod q\right\} /\left(\begin{array}{cc}1 & q \Lambda_{m} \\ 0 & 1_{m}\end{array}\right)$ correspond bijectively by $\sigma \mapsto(c, d)$, where we put $\widetilde{\sigma}=\left(\begin{array}{l}* \\ \tilde{c} \\ \tilde{d}\end{array}\right)$. The well-definedness is obvious. If $\left(c_{1}, d_{1}\right)=u\left(c_{2}, d_{2}\right)\left(\begin{array}{ll}1 & \lambda \\ 0 & 1\end{array}\right)$ for $u \in U_{m}(q), \lambda \in q \Lambda_{m}$ and $\sigma_{i}=\left(\begin{array}{cc}* & * \\ c_{i} & d_{i}\end{array}\right) \in \Gamma_{m}$, then $\sigma_{1}=\left(\begin{array}{ll}v & w \\ 0 & u\end{array}\right) \sigma_{2}\left(\begin{array}{ll}1 & \lambda \\ 0 & 1\end{array}\right)$ for $v \in U_{m}, w \in M_{m}$, and $\sigma_{1} \equiv \sigma_{2} \equiv \widetilde{\sigma} \bmod q \operatorname{implies}$ $\left(\begin{array}{ll}v & w \\ 0 & u\end{array}\right) \in \Gamma_{m}^{\infty}(q)$. Thus the mapping is injective. Conversely, suppose that $(c, d)$ are given in accordance with our conditions. Then there exist $\sigma=\left(\begin{array}{l}* * \\ c d\end{array}\right) \in \Gamma_{m}$. $\sigma \widetilde{\sigma}^{-1} \equiv\left(\begin{array}{cc}* & * \\ 0 & 1_{m}\end{array}\right) \bmod q$ yields that $\sigma \widetilde{\sigma}^{-1} \equiv\left(\begin{array}{ll}1 & \lambda \\ 0 & 1\end{array}\right) \bmod q$ for some $\lambda \in \Lambda_{m}$. Thus $\left(\begin{array}{cc}1 & -\lambda \\ 0 & 1\end{array}\right) \sigma=\left(\begin{array}{ll}* * \\ c & d\end{array}\right) \equiv \widetilde{\sigma} \bmod q$ and it means that the mapping is bijective. Thus rewriting the condition on $\sigma$ in terms of $c, d$, we have completed the proof.

\section{§3. Singular series}

We study the singular series on $c, d$ in the formula for $\mathcal{F}(T, y ; f, M)$ in Lemma 18. The first aim is to write it as a sum of infinite products. For $T \in \Lambda_{n}^{\prime}, \widetilde{\sigma} \in$ $\Gamma_{m}, \widetilde{v}_{2} \in M_{r, m}$, a positive integer $\alpha$, and $c, d \in M_{m}$ with $\operatorname{det} c \neq 0$, we put

$$
K_{\alpha}\left(T,(c, d) ; \widetilde{\sigma}, \widetilde{v}_{2}\right):=\sum_{\substack{v_{2} \in M_{r, m} \bmod \alpha M_{r, m} t_{c} \\
v_{2} \equiv \widetilde{v}_{2} \bmod \alpha}} e\left(\operatorname{tr} T\left(\begin{array}{cc}
a c^{-1}\left[{ }^{t} v_{2}\right] & -v_{2}{ }^{t} c^{-1} \\
-c^{-1 t} v_{2} & c^{-1} d
\end{array}\right) / \alpha\right),
$$

if there exist $a, b \in M_{m}$ such that $\Gamma_{m} \ni\left(\begin{array}{ll}a & b \\ c & d\end{array}\right) \equiv \widetilde{\sigma} \bmod \alpha$, and otherwise put $K_{\alpha}\left(T,(c, d) ; \widetilde{\sigma}, \widetilde{v}_{2}\right):=0$. The sum $K_{\alpha}$ is independent of the choice of $a$, since another 
$a$ is written as $a+\lambda c$ for $\lambda \in \alpha \Lambda_{m}$. It is clear that

$$
K_{q}\left(T,(c, d) ; \widetilde{\sigma}, \widetilde{v}_{2}\right)=K\left(T,(c, d), 1_{r} ; \widetilde{\sigma}, \widetilde{v}_{2}\right)
$$

When $\alpha=1, K_{\alpha}$ is independent of $\widetilde{\sigma}$ and $\widetilde{v}_{2}$ and so we put

$$
K(T,(c, d)):=K_{1}\left(T,(c, d) ; \widetilde{\sigma}, \widetilde{v}_{2}\right) .
$$

So, $K(T,(c, d))=0$ unless $\left(\begin{array}{c}* * \\ c d\end{array}\right) \in \Gamma_{m}$

LEMma 19. Let $\alpha_{i}, \beta_{i}(i=1,2)$ be natural numbers such that $\left(\beta_{1}, \beta_{2}\right)=1$ and $\alpha_{i} \mid \beta_{i}(i=1,2)$, and we decompose $c \in M_{m}$ with $\operatorname{det} c \neq 0$ as $c=u c_{1} c_{2} v$ where $u \in G L_{m}(\mathbf{Z}), v \in U_{m}$, and $c_{1}, c_{2}$ are diagonal matrices with $\mid \operatorname{det} c_{i} \| \beta_{i}(i=1,2)$. Put $D_{i}:=\left\{d_{i} \in M_{m} \bmod \alpha_{i} u c_{i} v \Lambda_{m} \mid\left(\begin{array}{cc}* & * \\ u c_{i} v & d_{i}\end{array}\right) \in \Gamma_{m}\right\}(i=1,2)$ and $D:=\{d \in$ $\left.M_{m} \bmod \alpha_{1} \alpha_{2} c \Lambda_{m} \mid\left(\begin{array}{c}* * \\ c d\end{array}\right) \in \Gamma_{m}\right\}$. We define a mapping $\phi$ from $D_{1} \times D_{2}$ to $D$ by $\phi\left(d_{1}, d_{2}\right):=\alpha_{2} u c_{2} u^{-1} d_{1}+\alpha_{1} u c_{1} u^{-1} d_{2}$, taking representatives $d_{1}, d_{2}$ such that $d_{1} \equiv 0 \bmod \beta_{2}$ and $d_{2} \equiv 0 \bmod \beta_{1}$. Then $\phi$ is bijective, and if $\left(\begin{array}{cc}a_{i} & b_{i} \\ c_{i} v & u^{-1} d_{i}\end{array}\right) \in \Gamma_{m}$, then there is an element $\sigma=\left(\begin{array}{cc}* & * \\ c \phi\left(d_{1}, d_{2}\right)\end{array}\right) \in \Gamma_{m}$ such that

$$
\sigma \equiv\left\{\begin{array}{l}
\left(\begin{array}{cc}
{ }^{t}\left(u c_{2}\right)^{-1} & \\
& u c_{2}
\end{array}\right)\left(\begin{array}{cc}
\alpha_{2}^{-1} a_{1} & b_{1} \\
c_{1} v & \alpha_{2} u^{-1} d_{1}
\end{array}\right) \bmod \beta_{1}, \\
\left(\begin{array}{cc}
{ }^{t}\left(u c_{1}\right)^{-1} & \\
& u c_{1}
\end{array}\right)\left(\begin{array}{cc}
\alpha_{1}^{-1} a_{2} & b_{2} \\
c_{2} v & \alpha_{1} u^{-1} d_{2}
\end{array}\right) \bmod \beta_{2} .
\end{array}\right.
$$

Proof. If $\left(\begin{array}{cc}* & * \\ u c_{1} v & d_{1}\end{array}\right) \in \Gamma_{m}$, then we can take $\lambda \in \Lambda_{m}$ such that $\alpha_{1} u c_{1} v \lambda+$ $d_{1} \equiv 0 \bmod \beta_{2}$ since $\left(\operatorname{det}\left(\alpha_{1} u c_{1} v\right), \beta_{2}\right)=1$ and $\alpha_{1} u c_{1} v^{t} d_{1} \in \Lambda_{m}$. Then $\left(\begin{array}{cc}* & * \\ u c_{1} v & d_{1}\end{array}\right)$ $\cdot\left(\begin{array}{cc}1 & \alpha_{1} \lambda \\ 0 & 1\end{array}\right)=\left(\begin{array}{cc}* & * \\ u c_{1} v & \alpha_{1} u c_{1} v \lambda+d_{1}\end{array}\right) \in \Lambda_{m}$. Hence we can choose representatives $d_{1}$ of $D_{1}$ such that $d_{1} \equiv 0 \bmod \beta_{2}$. Similarly we can choose $d_{2}$ with $d_{2} \equiv 0 \bmod \beta_{1}$ as representatives of $D_{2}$. Let us see that $\phi$ is well-defined. We have only to verify $\left(\begin{array}{ll}* & * \\ c \phi\left(d_{1}, d_{2}\right)\end{array}\right) \in$ $\Gamma_{m}$, which is equivalent to that $c^{t} \phi\left(d_{1}, d_{2}\right) \in \Lambda_{m}$ and $\left(c, \phi\left(d_{1}, d_{2}\right)\right)$ is primitive. It is easy to see $c^{t} \phi\left(d_{1}, d_{2}\right)=\alpha_{2} u c_{2} u^{-1}\left(u c_{1} v\right)^{t} d_{1}{ }^{t} u^{-1 t} c_{2}{ }^{t} u+\alpha_{1} u c_{1} u^{-1}\left(u c_{2} v\right)^{t} d_{2}{ }^{t} u^{-1 t}$ $c_{1}{ }^{t} u \in \Lambda_{m}$. Next let us see that $\left(c, \phi\left(d_{1}, d_{2}\right)\right)$ is primitive. To see this, we have only to see its validity over $\mathbf{Z} / p \mathbf{Z}$ for every prime $p$. It is clear if $p$ does not divide $\beta_{1} \beta_{2}$, since $\operatorname{det} c \not \equiv 0 \bmod p$. If $p \mid \beta_{2}$, then $\left(c, \phi\left(d_{1}, d_{2}\right)\right) \equiv\left(u c_{1} c_{2} v, \alpha_{1} u c_{1} u^{-1} d_{2}\right) \bmod$ $p \equiv u c_{1} u^{-1}\left(u c_{2} v, d_{2}\right)\left(\begin{array}{cc}1_{m} & \\ & \alpha_{1} 1_{m}\end{array}\right) \bmod p$ is primitive. If $p \mid \beta_{1}$, then $\left(c, \phi\left(d_{1}, d_{2}\right)\right) \equiv$ $\left(u c_{1} c_{2} v, \alpha_{2} u c_{2} u^{-1} d_{1}\right) \equiv u c_{2} u^{-1}\left(u c_{1} v, d_{1}\right)\left(\begin{array}{cc}1_{m} & \\ & \alpha_{2} 1_{m}\end{array}\right) \bmod p$ is also primitive. Thus $\phi$ is well-defined. Let us see $\phi$ is injective. Suppose that $d_{i}, d_{i}^{\prime} \in D_{i}(i=1,2)$ satisfy $\phi\left(d_{1}^{\prime}, d_{2}^{\prime}\right)=\phi\left(d_{1}, d_{2}\right)+\alpha_{1} \alpha_{2} c \lambda, \quad \lambda \in \Lambda_{m}$. Then $\lambda=\alpha_{1}^{-1} v^{-1} c_{1}^{-1} u^{-1}\left(d_{1}^{\prime}-\right.$ $\left.d_{1}\right)+\alpha_{2}^{-1} v^{-1} c_{2}^{-1} u^{-1}\left(d_{2}^{\prime}-d_{2}\right) \in \Lambda_{m}$, and prime divisors of denominators of entries 
of $\alpha_{i}^{-1} v^{-1} c_{i}^{-1} u^{-1}\left(d_{i}^{\prime}-d_{i}\right)$ divide $\beta_{i}(i=1,2)$. Hence $\alpha_{i}^{-1} v^{-1} c_{i}^{-1} u^{-1}\left(d_{i}^{\prime}-d_{i}\right) \in$ $\Lambda_{m}(i=1,2)$ follows and it means $d_{i}^{\prime}=d_{i}$ in $D_{i}$ for $i=1,2$. Thus $\phi$ is injective. Let us verify that $\phi$ is surjective. For this purpose, we choose integral diagonal matrices $f_{1}, f_{2}$ such that $f_{i} c_{i}(i=1,2)$ are scalar matrices and $\alpha_{1} c_{1} f_{1}+\alpha_{2} c_{2} f_{2}=$ $1_{m}$ and $f_{i} \equiv 0 \bmod \beta_{i}(i=1,2)$. We put $d_{1}=u f_{2} u^{-1} d, d_{2}=u f_{1} u^{-1} d$ for $d \in D$. Let us see $d_{i} \in D_{i}(i=1,2) . u c_{1} v^{t} d_{1}=\left(f_{2} c_{2}\right)^{-1}\left(u f_{2} u^{-1}\right) c^{t} d^{t}\left(u f_{2} u^{-1}\right)$, $u c_{2} v^{t} d_{2}=\left(f_{1} c_{1}\right)^{-1}\left(u f_{1} u^{-1}\right) c^{t} d^{t}\left(u f_{1} u^{-1}\right)$ are symmetric. If $p$ does not divide $\beta_{1}$, then $\left(u c_{1} v, d_{1}\right)$ is primitive over $\mathbf{Z} / p \mathbf{Z}$, and if $p \mid \beta_{1}$, then $\left(u c_{1} v, d_{1}\right)=\left(u f_{2} u^{-1}\right)(c, d)$ $\left(\begin{array}{cc}\left(c_{2} f_{2}\right)^{-1} & \\ & 1_{m}\end{array}\right)$ is primitive over $\mathbf{Z} / p \mathbf{Z}$. Thus $\left(u c_{1} v, d_{1}\right)$ and similarly $\left(u c_{2} v, d_{2}\right)$ are primitive and we have proved $d_{i} \in D_{i} . d_{1} \equiv 0 \bmod \beta_{2}$ and $d_{2} \equiv 0 \bmod \beta_{1}$ follow from the assumption, and $\phi\left(d_{1}, d_{2}\right)=d$ is clear. Therefore we have proved the surjectivity and so the bijectivity of $\phi$. Next, assume that $\left(\begin{array}{cc}a_{i} & b_{i} \\ c_{i} v & u^{-1} d_{i}\end{array}\right) \in \Gamma_{m}$ with $d_{1} \equiv 0 \bmod \beta_{2}, d_{2} \equiv 0 \bmod \beta_{1}$. Then $\left(c, \phi\left(d_{1}, d_{2}\right)\right) \equiv\left(u c_{1} c_{2} v, \alpha_{2} u c_{2} u^{-1} d_{1}\right) \bmod \beta_{1} \equiv$

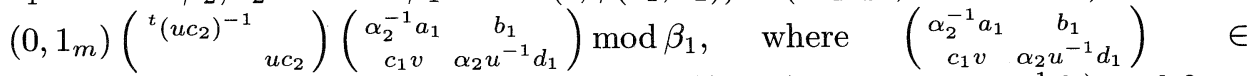
$S p\left(m, \mathbf{Z}_{p}\right)$ for $p \mid \beta_{1}$. We have also $\left(c, \phi\left(d_{1}, d_{2}\right)\right) \equiv\left(u c_{1} c_{2} v, \alpha_{1} u c_{1} u^{-1} d_{2}\right) \bmod \beta_{2} \equiv$

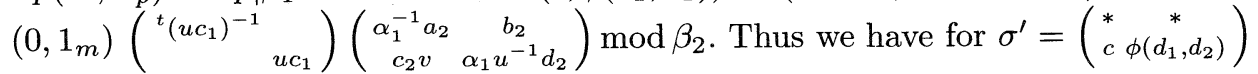
$\in \Gamma_{m}$

$$
\sigma^{\prime} \equiv\left\{\begin{array}{l}
\left(\begin{array}{cc}
1 & \lambda_{1} \\
0 & 1
\end{array}\right)\left(\begin{array}{cc}
{ }^{t}\left(u c_{2}\right)^{-1} & \\
& u c_{2}
\end{array}\right)\left(\begin{array}{cc}
\alpha_{2}^{-1} a_{1} & b_{1} \\
c_{1} v & \alpha_{2} u^{-1} d_{1}
\end{array}\right) \bmod \beta_{1} \\
\left(\begin{array}{cc}
1 & \lambda_{2} \\
0 & 1
\end{array}\right)\left(\begin{array}{cc}
{ }^{t}\left(u c_{1}\right)^{-1} & \\
& u c_{1}
\end{array}\right)\left(\begin{array}{cc}
\alpha_{1}^{-1} a_{2} & b_{2} \\
c_{2} v & \alpha_{1} u^{-1} d_{2}
\end{array}\right) \bmod \beta_{2} .
\end{array}\right.
$$

for $\lambda_{i} \in \Lambda_{m}$. Taking $\lambda \in \Lambda_{m}$ such that $\lambda \equiv \lambda_{i} \bmod \beta_{i}, \sigma=\left(\begin{array}{cc}1 & -\lambda \\ 0 & 1\end{array}\right) \sigma^{\prime}$ is what we want.

LEMMA 20. Let $\alpha_{i}, \beta_{i}$ be natural numbers such that $\alpha_{1} \alpha_{2}=q, \alpha_{i} \mid \beta_{i}$ and $\left(\beta_{1}, \beta_{2}\right)=1$. Let $c \in M_{m}$ satisfy that $\operatorname{det} c \neq 0$ and $\operatorname{det} c \mid\left(\beta_{1} \beta_{2}\right)^{\infty}$. Write $c=u c_{1} c_{2} v$, where $u \in G L_{m}(\mathbf{Z}), v \in U_{m}$ and $c_{i}$ is diagonal such that $\operatorname{det} c_{i} \mid \beta_{i}^{\infty}(i=1,2)$. If $\left(\begin{array}{cc}a_{i} & b_{i} \\ c_{i} v u^{-1} d_{i}\end{array}\right) \in \Gamma_{m}(i=1,2)$ with $d_{1} \equiv 0 \bmod \left(\beta_{2} \operatorname{det} c_{2}\right), d_{2} \equiv 0 \bmod \left(\beta_{1} \operatorname{det} c_{1}\right)$, then we have

$$
\begin{aligned}
& K_{q}\left(T,\left(u c_{1} c_{2} v, \alpha_{2} u c_{2} u^{-1} d_{1}+\alpha_{1} u c_{1} u^{-1} d_{2}\right) ; \widetilde{\sigma}, \widetilde{v}_{2}\right) \\
& =K_{\alpha_{2}}\left(T,\left(c_{2} v, u^{-1} d_{2}\right) ;\left[\alpha_{1} \ddagger\left({ }^{t}\left(u c_{1}\right)_{\left(u c_{1}\right)^{-1}}\right) \sigma\right]_{\alpha_{2}}, \alpha_{1}^{-1} \widetilde{v}_{2}{ }^{t} u^{-1} c_{1}^{-1}\right) \\
& \quad \times K_{\alpha_{1}}\left(T,\left(c_{1} v, u^{-1} d_{1}\right),\left[\alpha_{2} \ddagger\left({ }^{t}\left(u c_{2}\right)_{\left(u c_{2}\right)^{-1}}\right) \sigma\right]_{\alpha_{1}}, \alpha_{2}^{-1} \widetilde{v}_{2}{ }^{t} u^{-1} c_{2}^{-1}\right)
\end{aligned}
$$

where $\left[\alpha \ddagger\left(\begin{array}{ll}a & b \\ c & d\end{array}\right)\right]_{\beta}$ stands for an element of $\Gamma_{m}$ which is congruent to $\left(\begin{array}{cc}\alpha a & b \\ c & \alpha^{-1} d\end{array}\right)$ modulo $\beta$. 
Proof. By virtue of Lemma 19, there exist $\sigma=\left(\begin{array}{ll}a & b \\ c & d\end{array}\right) \in \Gamma_{m}$ with $d:=$ $\alpha_{2} u c_{2} u^{-1} d_{1}+\alpha_{1} u c_{1} u^{-1} d_{2}$ such that

$$
\sigma \equiv\left\{\begin{array}{l}
\left(\begin{array}{ll}
{ }^{t}\left(u c_{2}\right)^{-1} & \\
& u c_{2}
\end{array}\right)\left(\begin{array}{cc}
\alpha_{2}^{-1} a_{1} & b_{1} \\
c_{1} v & \alpha_{2} u^{-1} d_{1}
\end{array}\right) \bmod \beta_{1} \operatorname{det} c_{1} \\
\left(\begin{array}{cc}
{ }^{t}\left(u c_{1}\right)^{-1} & \\
& u c_{1}
\end{array}\right)\left(\begin{array}{cc}
\alpha_{1}^{-1} a_{2} & b_{2} \\
c_{2} v & \alpha_{1} u^{-1} d_{2}
\end{array}\right) \bmod \beta_{2} \operatorname{det} c_{2} .
\end{array}\right.
$$

If there is an element $\left(\begin{array}{ll}* * & * \\ c & d\end{array}\right)=\left(\begin{array}{ll}1 & \lambda \\ 0 & 1\end{array}\right) \sigma \in \Gamma_{m}$ such that $\left(\begin{array}{ll}1 & \lambda \\ 0 & 1\end{array}\right) \sigma \equiv \widetilde{\sigma} \bmod q$, then

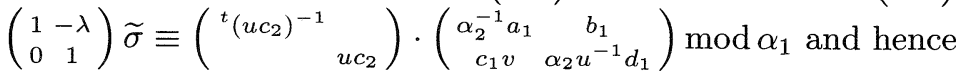

$$
\begin{aligned}
& {\left[\alpha_{2} \ddagger\left({ }^{t}\left(u c_{2}\right)_{\left(u c_{2}\right)^{-1}}\right) \tilde{\sigma}\right]_{\alpha_{1}}} \\
& \equiv\left(\begin{array}{cc}
1 & \alpha_{2} \lambda\left[u c_{2}\right] \\
0 & 1
\end{array}\right)\left(\begin{array}{cc}
a_{1} & b_{1} \\
c_{1} v & u^{-1} d_{1}
\end{array}\right) \bmod \alpha_{1} \equiv\left(\begin{array}{cc}
* & -* \\
c_{1} v & u^{-1} d_{1}
\end{array}\right) \bmod \alpha_{1}
\end{aligned}
$$

Similarly we have

$$
\begin{aligned}
& {\left[\alpha_{1} \ddagger\left({ }^{t}\left(u c_{1}\right)_{\left(u c_{1}\right)^{-1}}\right) \tilde{\sigma}\right]_{\alpha_{2}}} \\
& \equiv\left(\begin{array}{cc}
1 & \alpha_{1} \lambda\left[u c_{1}\right] \\
0 & 1
\end{array}\right)\left(\begin{array}{cc}
a_{2} & b_{2} \\
c_{2} v & u^{-1} d_{2}
\end{array}\right) \bmod \alpha_{2} \equiv\left(\begin{array}{cc}
* & -* \\
c_{2} v & u^{-1} d_{2}
\end{array}\right) \bmod \alpha_{2} \text {. }
\end{aligned}
$$

If conversely $\left.\left[\begin{array}{lll}\alpha_{2} \ddagger\left({ }^{t}\left(u c_{2}\right)\right. & \\ & \left(u c_{2}\right)^{-1}\end{array}\right) \widetilde{\sigma}\right]_{\alpha_{1}} \equiv\left(\begin{array}{cc}a_{1}^{\prime} & b_{1}^{\prime} \\ c_{1} v & u^{-1} d_{1}\end{array}\right) \bmod \alpha_{1},\left[\begin{array}{ll}\alpha_{1} \ddagger\left({ }^{t}\left(u c_{1}\right)\right. & \\ & \left(u c_{1}\right)^{-1}\end{array}\right)$ $\tilde{\sigma}]_{\alpha_{2}} \equiv\left(\begin{array}{cc}a_{2}^{\prime} & b_{2}^{\prime} \\ c_{2} v & u^{-1} d_{2}\end{array}\right) \bmod \alpha_{2}$ for $\left(\begin{array}{cc}a_{i}^{\prime} & b_{i}^{\prime} \\ c_{i} v & u^{-1} d_{i}\end{array}\right) \in \Gamma_{m}$, then taking $\lambda_{i} \in \Lambda_{m}$ such that $\left(\begin{array}{cc}a_{i}^{\prime} & b_{i}^{\prime} \\ c_{i} v & u^{-1} d_{i}\end{array}\right)=\left(\begin{array}{cc}1 & \lambda_{i} \\ 0 & 1\end{array}\right)\left(\begin{array}{cc}a_{i} & b_{i} \\ c_{i} v & u^{-1} d_{i}\end{array}\right)$, we choose $\lambda \in \Lambda_{m}$ such that $\alpha_{2} \lambda\left[u c_{2}\right] \equiv$ $\lambda_{1} \bmod \alpha_{1}, \alpha_{1} \lambda\left[u c_{1}\right] \equiv \lambda_{2} \bmod \alpha_{2}$. Then we have $\left(\begin{array}{ll}1 & \lambda \\ 0 & 1\end{array}\right) \sigma \equiv \widetilde{\sigma} \bmod q$. Thus $(c, d)$ has an extension to $\Gamma_{m}$ which is congruent to $\widetilde{\sigma}$ modulo $q$ if and only if $\left(c_{1} v, u^{-1} d_{1}\right)\left(\operatorname{resp} .\left(c_{2} v, u^{-1} d_{2}\right)\right)$ has an extension to $\Gamma_{m}$ which is congruent to $\left[\alpha_{2} \ddagger\left({ }^{t}\left(u c_{2}\right){ }_{\left(u c_{2}\right)^{-1}}\right) \tilde{\sigma}\right]_{\alpha_{1}}$ modulo $\alpha_{1}$ (resp. $\left[\alpha_{1} \ddagger\left({ }^{t}\left(u c_{1}\right)_{\left(u c_{1}\right)^{-1}}\right) \tilde{\sigma}\right]_{\alpha_{2}}$ modulo $\left.\alpha_{2}\right)$. Hence $K_{q}=K_{\alpha_{1}}=K_{\alpha_{2}}=0$ if there are no such extensions.

Now we may suppose that $(c, d)$ has an extension to $\Gamma_{m}$ which is congruent to $\widetilde{\sigma} \bmod q$. Let it be $\left(\begin{array}{ll}1 & \lambda \\ 0 & 1\end{array}\right) \sigma$ for $\lambda \in \Lambda_{m}$ and $\sigma=\left(\begin{array}{ll}a & b \\ c & d\end{array}\right)$ satisfies (1). Now we have $K_{q}:=K_{q}\left(T,\left(u c_{1} c_{2} v, \alpha_{2} u c_{2} u^{-1} d_{1}+\alpha_{1} u c_{1} u^{-1} d_{2}\right) ; \widetilde{\sigma}, \widetilde{v}_{2}\right)=\sum V_{V_{2} \in M_{r, m} \bmod \alpha_{1} \alpha_{2} M_{r, m} t_{c}}$ $e\left(\operatorname{tr} T\left(\begin{array}{c}(a+\lambda c)\left(u c_{1} c_{2} v\right)^{-1}\left[{ }^{t} V_{2}\right] \\ -\left(u c_{1} c_{2} v\right)^{-1 t} V_{2}\end{array} \alpha_{2} v^{-1} c_{1}^{-1} u^{-1} d_{1}+\alpha_{1} v^{-1} c_{2}^{-1} u^{-1} d_{2}\right) /\left(\alpha_{1} \alpha_{2}\right)\right)$. Now we take integers $f_{1}, f_{2}$ such that $\alpha_{1} f_{1}\left(\operatorname{det} c_{1}\right)^{2}+\alpha_{2} f_{2}\left(\operatorname{det} c_{2}\right)^{2}=1$.

Let us see that $V_{2} \in M_{r, m} \bmod \alpha_{1} \alpha_{2} M_{r, m}{ }^{t} c$ with $V_{2} \equiv \widetilde{v}_{2} \bmod \alpha_{1} \alpha_{2}$ can be written as

$$
V_{2}=\left(\alpha_{1} x c_{1}+\alpha_{2} y c_{2}\right)^{t} u
$$


where $x \in M_{r, m} \bmod \alpha_{2} M_{r, m} c_{2}$ with $\alpha_{1} x c_{1} \equiv \widetilde{v}_{2}{ }^{t} u^{-1} \bmod \alpha_{2}$, and $y \in M_{r, m}$ $\bmod \alpha_{1} M_{r, m} c_{1}$ with $\alpha_{2} y c_{2} \equiv \widetilde{v}_{2}^{t} u^{-1} \bmod \alpha_{1}$. Suppose that $V_{2}$ is given with $V_{2} \equiv$ $\widetilde{v}_{2} \bmod \alpha_{1} \alpha_{2}$. Putting $x=f_{1} \operatorname{det} c_{1}^{2} V_{2}{ }^{t} u^{-1} c_{1}^{-1}, y=f_{2} \operatorname{det} c_{2}^{2} V_{2}{ }^{t} u^{-1} c_{2}^{-1}$, we have $V_{2}{ }^{t} u^{-1}=\alpha_{1} x c_{1}+\alpha_{2} y c_{2}$, and $V_{2} \equiv \widetilde{v}_{2} \bmod \alpha_{1} \alpha_{2}$ if and only if $\alpha_{2} y c_{2} \equiv \widetilde{v}_{2}{ }^{t} u^{-1}$ $\bmod \alpha_{1}$ and $\alpha_{1} x c_{1} \equiv \widetilde{v}_{2}{ }^{t} u^{-1} \bmod \alpha_{2} . V_{2} \in \alpha_{1} \alpha_{2} M_{r, m}{ }^{t} c$ if and only if $V_{2}{ }^{t} u^{-1}$ $\in \alpha_{1} \alpha_{2} M_{r, m} c_{1} c_{2}$, which implies $\alpha_{1} x c_{1} \in \alpha_{2} M_{r, m} c_{2}$ and $\alpha_{2} y c_{2} \in \alpha_{1} M_{r, m} c_{1} .\left(\alpha_{1} \times\right.$ $\left.\operatorname{det} c_{1}, \alpha_{2} \operatorname{det} c_{2}\right)=1$ yields then $x \in \alpha_{2} M_{r, m} c_{2}$ and $y_{2} \in \alpha_{1} M_{r, m} c_{1}$. Conversely they imply $V_{2}=\left(\alpha_{1} x c_{1}+\alpha_{2} y c_{2}\right)^{t} u \in \alpha_{1} \alpha_{2} M_{r, m}{ }^{t} c$. Thus the validity of the decomposition has been proved.

For the above decomposition $V_{2}=\left(\alpha_{1} x c_{1}+\alpha_{2} y c_{2}\right)^{t} u$, we have $(a+\lambda c)$ $\left(u c_{1} c_{2} v\right)^{-1}\left[{ }^{t} V_{2}\right]=a v^{-1} c_{2}^{-1} c_{1}^{-1} u^{-1}\left[u\left(\alpha_{1} c_{1}{ }^{t} x+\alpha_{2} c_{2}{ }^{t} y\right)\right]+\lambda\left[{ }^{t} V_{2}\right]=\alpha_{1}^{2} x c_{1}{ }^{t} u a v^{-1} c_{2}^{-1 t}$ $x+\alpha_{1} \alpha_{2} x c_{1}{ }^{t} u a v^{-1} c_{1}^{-1 t} y+\alpha_{1} \alpha_{2} y c_{1}^{-1 t} v^{-1 t} a u c_{1}{ }^{t} x+\alpha_{2}^{2} y c_{2}{ }^{t} u a v^{-1} c_{1}^{-1 t} y+\lambda\left[{ }^{t} V_{2}\right]$ $\equiv \alpha_{1}\left(a_{2}\left(c_{2} v\right)^{-1}\right)\left[{ }^{t} x\right]+\alpha_{2}\left(a_{1}\left(c_{1} v\right)^{-1}\right)\left[{ }^{t} y\right]+\lambda\left[{ }^{t} V_{2}\right] \bmod q$ by (1), $\alpha_{1} \alpha_{2}=q, \alpha_{i} \mid \beta_{i}$. $\left(u c_{1} c_{2} v\right)^{-1 t} V_{2}=\alpha_{1} v^{-1} c_{2}^{-1 t} x+\alpha_{2} v^{-1} c_{1}^{-1 t} y$ is clear. Thus we have

$$
\begin{aligned}
& K_{q}\left(T,\left(u c_{1} c_{2} v, \alpha_{2} u c_{2} u^{-1} d_{1}+\alpha_{1} u c_{1} u^{-1} d_{2}\right) ; \widetilde{\sigma}, \widetilde{v}_{2}\right) \\
& =\sum_{x} e\left(\operatorname{tr} T\left(\begin{array}{cc}
\left(a_{2}+\alpha_{1} \lambda\left[u c_{1}\right] c_{2} v\right)\left(c_{2} v\right)^{-1}\left[{ }^{t} x\right] & * \\
-\left(c_{2} v\right)^{-1 t} x & * \\
\left(c_{2} v\right)^{-1} u^{-1} d_{2}
\end{array}\right) / \alpha_{2}\right) \\
& \times \sum_{y} e\left(\operatorname{tr} T\left(\begin{array}{cc}
\left(a_{1}+\alpha_{2} \lambda\left[u c_{2}\right] c_{1} v\right)\left(c_{1} v\right)^{-1}\left[{ }^{t} y\right] & * \\
-\left(c_{1} v\right)^{-1} y & * \\
\left(c_{1} v\right)^{-1} u^{-1} d_{1}
\end{array}\right) / \alpha_{1}\right)
\end{aligned}
$$

where $x$ runs over $M_{r, m} \bmod \alpha_{2} M_{r, m} c_{2}$ with $\alpha_{1} x c_{1} \equiv \widetilde{v}_{2}{ }^{t} u^{-1} \bmod \alpha_{2}, y$ runs over $M_{r, m} \bmod \alpha_{1} M_{r, m} c_{1}$ with $\alpha_{2} y c_{2} \equiv \widetilde{v}_{2}{ }^{t} u^{-1} \bmod \alpha_{1}$, and this is equal to

$$
\begin{aligned}
& K_{\alpha_{2}}\left(T,\left(c_{2} v, u^{-1} d_{2}\right) ;\left(\begin{array}{cc}
1 & \alpha_{1} \lambda\left[u c_{1}\right] \\
0 & 1
\end{array}\right)\left(\begin{array}{cc}
a_{2} & b_{2} \\
c_{2} v & u^{-1} d_{2}
\end{array}\right), \alpha_{1}^{-1} \widetilde{v}_{2}^{t} u^{-1} c_{1}^{-1}\right) \\
& \times K_{\alpha_{1}}\left(T,\left(c_{1} v, u^{-1} d_{1}\right) ;\left(\begin{array}{cc}
1 & \alpha_{2} \lambda\left[u c_{2}\right] \\
0 & 1
\end{array}\right)\left(\begin{array}{cc}
a_{1} & b_{1} \\
c_{1} v & u^{-1} d_{1}
\end{array}\right), \alpha_{2}^{-1} \widetilde{v}_{2}^{t} u^{-1} c_{2}^{-1}\right),
\end{aligned}
$$

which completes the proof by (2) and (3).

LEMMA 21. Let $\alpha_{1}, \alpha_{2}$ be relatively prime natural numbers. We decompose $c \in M_{m}$ with $\operatorname{det} c \neq 0$ as $c=u c_{1} c_{2} v_{1} v_{2}$ where $u \in G L_{m}(\mathbf{Z}), v_{1} \in c_{2}^{-1} U_{m} c_{2} \cap$ $U_{m}, v_{2} \in U_{m}\left(\alpha_{1}\right),\left(\alpha_{1} \operatorname{det} c_{1}, \alpha_{2} \operatorname{det} c_{2}\right)=1$. Then

$$
\begin{aligned}
& \sum_{d \bmod \alpha_{1} \alpha_{2} c \Lambda_{m}} K_{\alpha_{1} \alpha_{2}}\left(T,(c, d) ; \widetilde{\sigma}, \widetilde{v}_{2}\right) \\
& =\sum_{d_{1} \bmod \alpha_{1} c_{1} v_{1} \Lambda_{m}} K_{\alpha_{1}}\left(T,\left(c_{1} v_{1}, d_{1}\right) ;\left[\alpha_{2} \ddagger\left({ }^{t}\left(u c_{2}\right){ }_{\left(u c_{2}\right)^{-1}}\right) \widetilde{\sigma}\right]_{\alpha_{1}}, \alpha_{2}^{-1} \widetilde{v}_{2}{ }^{t}\left(u c_{2}\right)^{-1}\right) \\
& \times \sum_{d_{2} \bmod \alpha_{2} c_{2} v_{2} \Lambda_{m}} K_{\alpha_{2}}\left(T,\left(c_{2} v_{2}, d_{2}\right) ;\left[\alpha_{1} \ddagger\left({ }^{t}\left(u c_{1} x\right){ }_{\left(u c_{1} x\right)^{-1}}\right) \widetilde{\sigma}\right]_{\alpha_{2}}, \alpha_{1}^{-1} \widetilde{v}_{2}{ }^{t}\left(u c_{1} x\right)^{-1}\right)
\end{aligned}
$$


where $x:=c_{2} v_{1} c_{2}^{-1} \in U_{m}$.

Proof. Put $\beta_{i}=\alpha_{i} \operatorname{det} c_{i}(i=1,2)$; then we have $\left(\beta_{1}, \beta_{2}\right)=1$. Then by Lemmas 19, 20 we have, putting $v:=v_{1} v_{2}$

$$
\begin{aligned}
& \sum_{d \bmod \alpha_{1} \alpha_{2} c \Lambda_{m}} K_{\alpha_{1} \alpha_{2}}\left(T,(c, d) ; \tilde{\sigma}, \widetilde{v}_{2}\right) \\
= & \sum_{d_{1} \bmod \alpha_{1} u c_{1} v \Lambda_{m}} K_{\alpha_{1}}\left(T,\left(c_{1} v, u^{-1} d_{1}\right) ;\left[\alpha_{2} \ddagger\left({ }^{t}\left(u c_{2}\right)_{\left(u c_{2}\right)^{-1}}\right) \widetilde{\sigma}\right]_{\alpha_{1}}, \alpha_{2}^{-1} \widetilde{v}_{2}{ }^{t}\left(u c_{2}\right)^{-1}\right) \\
\times & \sum_{d_{2} \bmod \alpha_{2} u c_{2} v \Lambda_{m}} K_{\alpha_{2}}\left(T,\left(c_{2} v, u^{-1} d_{2}\right) ;\left[\alpha_{1} \ddagger\left({ }^{t}\left(u c_{1}\right)_{\left(u c_{1}\right)^{-1}}\right) \widetilde{\sigma}\right]_{\alpha_{2}}, \alpha_{1}^{-1} \widetilde{v}_{2}{ }^{t}\left(u c_{1}\right)^{-1}\right) .
\end{aligned}
$$

Put $u^{-1} d_{1}=D_{1}{ }^{t} v_{2}^{-1}$. Then $D_{1}$ runs over $M_{m} \bmod \alpha_{1} c_{1} v_{1} \Lambda_{m}$ and $v_{2} \equiv 1 \bmod \alpha_{1}$ yields that the sum on $d_{1}$ above is equal to the sum on $d_{1}$ in the assertion of Lemma by (2) in Lemma 11. $x:=c_{2} v_{1} c_{2}^{-1} \in U_{m}$ implies $c_{2} v=x c_{2} v_{2}$, and then the condition $d_{2} \in M_{m} \bmod \alpha_{2} u c_{2} v \Lambda_{m}$ is equivalent to $u^{-1} d_{2} \in M_{m} \bmod \alpha_{2} x c_{2} v_{2} \Lambda_{m}$. Thus putting $u^{-1} d_{2}=x D_{2}$, we get the sum on $d_{2}$ above to be equal to $\sum_{D_{2} \in M_{m} \bmod \alpha_{2} c_{2} v_{2} \Lambda_{m}} K_{\alpha_{2}}\left(T, x\left(c_{2} v_{2}, D_{2}\right) ;\left[\alpha_{1} \ddagger\left({ }^{t}\left(u c_{1}\right){ }_{\left(u c_{1}\right)^{-1}}\right) \tilde{\sigma}\right]_{\alpha_{2}}, \alpha_{1}^{-1}\right.$ $\left.\widetilde{v}_{2}{ }^{t}\left(u c_{1}\right)^{-1}\right)$. Applying (2) in Lemma 11 for $u=x, \hat{u}=\hat{v}=0, v=1_{m}$ to this sum, we see that it is the same as the sum on $d_{2}$ in the equality to be proved.

Lemma 22. If $c_{i} \in M_{m}(i=1,2)$ satisfies $c_{1} c_{2}=c_{2} c_{1}$, $\operatorname{det}\left(c_{1} c_{2}\right) \neq 0$, and $\left(\operatorname{det} c_{1}, \operatorname{det} c_{2}\right)=1$. Then we have

(i) $\left(c_{1} c_{2}\right)^{-1} U_{m} c_{1} c_{2} \cap U_{m}=c_{1}^{-1} U_{m} c_{1} \cap c_{2}^{-1} U_{m} c_{2} \cap U_{m}$,

(ii) a complete set of representatives of $\left(\left(c_{1} c_{2}\right)^{-1} U_{m}\left(c_{1} c_{2}\right) \cap U_{m}\right) \backslash\left(c_{2}^{-1} U_{m} c_{2} \cap U_{m}\right)$ becomes a complete set of representatives of $\left(c_{1}^{-1} U_{m} c_{1} \cap U_{m}\right) \backslash U_{m}$,

(iii) a complete set of representatives of $G L_{m}(\mathbf{Z}) \backslash G L_{m}(\mathbf{Z}) c_{1} c_{2} G L_{m}(\mathbf{Z})$ is given by $c_{1} c_{2} v_{1} v_{2}$, where

$$
\begin{aligned}
& v_{1} \in\left(\left(c_{1} c_{2}\right)^{-1} U_{m} c_{1} c_{2} \cap U_{m}\right) \backslash\left(c_{2}^{-1} U_{m} c_{2} \cap U_{m}\right) \\
& v_{2} \in\left(c_{2}^{-1} U_{m} c_{2} \cap U_{m}\right) \backslash U_{m} \quad \text { with } v_{2} \equiv 1_{m} \bmod \alpha,
\end{aligned}
$$

where $\alpha$ is any given natural number relatively prime to $\operatorname{det} c_{2}$.

Proof. (i) If $g=\left(c_{1} c_{2}\right)^{-1} g^{\prime}\left(c_{1} c_{2}\right)$ for $g, g^{\prime} \in U_{m}$, then $c_{1} g c_{1}^{-1}=c_{2}^{-1} g^{\prime} c_{2}$ follows, and denominators of entries of both sides are relatively prime. Hence $c_{1} g c_{1}^{-1}=c_{2}^{-1} g^{\prime} c_{2} \in M_{m}$. Then $g \in c_{1}^{-1} M_{m} c_{1}$, and similarly we get $g \in c_{2}^{-1} M_{m} c_{2}$ using $g=\left(c_{2} c_{1}\right)^{-1} g^{\prime}\left(c_{2} c_{1}\right)$. So the left hand side of (i) is contained in the right hand side. If $g=c_{i}^{-1} g_{i} c_{i}$ for $g_{i} \in U_{m}(i=1,2)$, then we have $\left(c_{1} c_{2}\right) g\left(c_{1} c_{2}\right)^{-1}=$ $c_{1} g_{2} c_{1}^{-1}=c_{2} g_{1} c_{2}^{-1} \cdot c_{1} g_{2} c_{1}^{-1} \in\left(\operatorname{det} c_{1}\right)^{-1} M_{m}$ and $c_{2} g_{1} c_{2}^{-1} \in\left(\operatorname{det} c_{2}\right)^{-1} M_{m}$ yield $\left(c_{1} c_{2}\right) g\left(c_{1} c_{2}\right)^{-1} \in M_{m}$. Thus the right hand side is in the left hand side. 
(ii) Suppose that $g_{i} \in c_{2}^{-1} U_{m} c_{2} \cap U_{m}(i=1,2)$ satisfies $\left(c_{1}^{-1} U_{m} c_{1} \cap U_{m}\right) g_{1}=$ $\left(c_{1}^{-1} U_{m} c_{1} \cap U_{m}\right) g_{2}$. Then $g_{1} g_{2}^{-1} \in c_{1}^{-1} U_{m} c_{1} \cap U_{m} \cap c_{2}^{-1} U_{m} c_{2}=\left(c_{1} c_{2}\right)^{-1} U_{m} c_{1} c_{2} \cap$ $U_{m}$ by (i). It remains to show that we can choose elements of $c_{2}^{-1} U_{m} c_{2} \cap U_{m}$ as representatives of $\left(c_{1}^{-1} U_{m} c_{1} \cap U_{m}\right) \backslash U_{m}$. Take any element $g \in U_{m}$ and choose an element $v \in U_{m}$ by $v \equiv 1_{m} \bmod \operatorname{det} c_{1}$ and $v \equiv g^{-1} \bmod \operatorname{det} c_{2}$. Putting $u:=v g$, we have $c_{2} u c_{2}^{-1}=c_{2} v g c_{2}^{-1} \in M_{m}$ because of $v \equiv g^{-1} \bmod \operatorname{det} c_{2}$. These imply $u \in c_{2}^{-1} U_{m} c_{2} \cap U_{m}$ and $v \in c_{1}^{-1} U_{m} c_{1} \cap U_{m}$, which are what we want.

(iii) is clear except for condition $v_{2} \equiv 1_{m} \bmod \alpha$. It is enough to show that for a given $g \in U_{m}$, there exists $u \in U_{m}(\alpha)$ such that $u \in\left(c_{2}^{-1} U_{m} c_{2} \cap U_{m}\right) g$. Take $v \in U_{m}$ such that $v \equiv 1_{m} \bmod \operatorname{det} c_{2}, v \equiv g^{-1} \bmod \alpha$. Then $v$ satisfies $v \in c_{2}^{-1} U_{m} c_{2} \cap U_{m}$ and $u:=v g \equiv 1_{m} \bmod \alpha$. Therefore $u$ is the required one.

Lemma 23. Let $C_{i}(i=1,2)$ be integral and diagonal regular matrix in $M_{m}$ such that $\left(\operatorname{det} C_{1}, \operatorname{det} C_{2}\right)=1$. For natural numbers $\alpha_{1}, \alpha_{2}$ with $\left(\alpha_{1} \operatorname{det} C_{1}, \alpha_{2} \operatorname{det}\right.$ $\left.C_{2}\right)=1$, we have

$$
\begin{aligned}
& \sum_{c \in U_{m}\left(\alpha_{1} \alpha_{2}\right) \backslash U_{m} C_{1} C_{2} U_{m}} \sum_{d \in M_{m}} K_{\bmod \alpha_{1} \alpha_{2} c \Lambda_{m}} K_{\alpha_{1} \alpha_{2}}\left(T,(c, d) ; \tilde{\sigma}, \widetilde{v}_{2}\right) \\
& =\sum_{c_{1}} \sum_{d_{1}} K_{\alpha_{1}}\left(T,\left(c_{1}, d_{1}\right) ;\left[\alpha_{2} \ddagger\left(\widehat{\frac{\operatorname{det} C_{2}}{\widehat{\operatorname{det} C}_{2}^{-1}}}\right) \widetilde{\sigma}_{\alpha_{1}}, \alpha_{2}^{-1} \widetilde{v}_{2}{\widehat{\operatorname{det} C_{2}}}^{-1}\right)\right.
\end{aligned}
$$

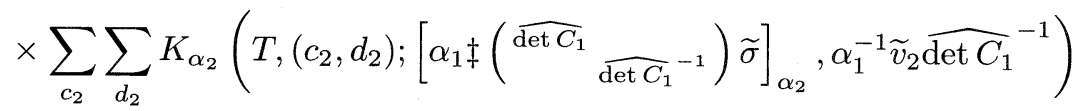

where $c_{i}, d_{i}$ run over $U_{m}\left(\alpha_{i}\right) \backslash U_{m} C_{i} U_{m}, M_{m} \bmod \alpha_{i} c_{i} \Lambda_{m}$ respectively, and $\widehat{\operatorname{det} C_{i}}$ stands for $\operatorname{diag}\left(1_{m-1}, \operatorname{det} C_{i}\right)$.

Proof. In the assertion of Lemma 21, putting $u=u_{1} u_{2}$ where $u_{i} \equiv 1_{m} \bmod$ $\alpha_{i}, u_{i} \in U_{m}$, we have

$$
\begin{aligned}
& \sum_{d \bmod \alpha_{1} \alpha_{2} u c_{1} c_{2} v_{1} v_{2} \Lambda_{m}} K_{\alpha_{1} \alpha_{2}}\left(T,\left(u_{1} u_{2} c_{1} c_{2} v_{1} v_{2}, d\right) ; \widetilde{\sigma}, \widetilde{v}_{2}\right) \\
= & \sum_{d_{1}} K_{\alpha_{1}}\left(T,\left(c_{1} v_{1}, d_{1}\right) ;\left[\alpha_{2} \ddagger\left({ }^{t}\left(u_{2} c_{2}\right)_{\left(u_{2} c_{2}\right)^{-1}}\right) \widetilde{\sigma}\right]_{\alpha_{1}}, \alpha_{2}^{-1} \widetilde{v}_{2}{ }^{t}\left(u_{2} c_{2}\right)^{-1}\right) \\
\times & \sum_{d_{2}} K_{\alpha_{2}}\left(T,\left(c_{2} v_{2}, d_{2}\right) ;\left[\alpha_{1} \ddagger\left({ }^{t}\left(u_{1} c_{1} x\right)_{\left(u_{1} c_{1} x\right)^{-1}}\right) \tilde{\sigma}\right]_{\alpha_{2}}, \alpha_{1}^{-1} \widetilde{v}_{2}{ }^{t}\left(u_{1} c_{1} x\right)^{-1}\right)
\end{aligned}
$$

where $x=c_{2} v_{1} c_{2}^{-1} \in U_{m}$ and $d_{i}$ runs over $M_{m} \bmod \alpha_{i} c_{i} v_{i} \Lambda_{m}$. Note that if $u_{1}$ runs over $U_{m}\left(\alpha_{1} \alpha_{2}\right) \backslash U_{m}\left(\alpha_{1}\right)$, then $u_{1} c_{1} x$ runs over $\left\{y \in M_{m} \bmod \alpha_{2} M_{m} \mid \operatorname{det} y \equiv\right.$ $\left.\operatorname{det} c_{1} \bmod \alpha_{2}\right\}$, and if $u_{2}$ runs over $U_{m}\left(\alpha_{1} \alpha_{2}\right) \backslash U_{m}\left(\alpha_{2}\right)$, then $u_{2} c_{2}$ runs over $\{z \in$ $\left.M_{m} \bmod \alpha_{1} M_{m} \mid \operatorname{det} z \equiv \operatorname{det} c_{2} \bmod \alpha_{1}\right\}$. Hence, if $u=u_{1} u_{2}$ runs over $U_{m}\left(\alpha_{1} \alpha_{2}\right) \backslash$ 
$U_{m}$ as above, then $u_{2} c_{2}$ and $u_{1} c_{1} x$ in (1) runs over $\left\{z \in M_{m} \bmod \alpha_{1} M_{m} \mid \operatorname{det} z \equiv\right.$ $\left.\operatorname{det} c_{2} \bmod \alpha_{1}\right\}$ and $\left\{y \in M_{m} \bmod \alpha_{2} M_{m} \mid \operatorname{det} y \equiv \operatorname{det} c_{1} \bmod \alpha_{2}\right\}$ respectively. Now adding (1) on $u=u_{1} u_{2} \in U_{m}\left(\alpha_{1} \alpha_{2}\right) \backslash U_{m}$, we have

$$
\begin{aligned}
& \sum_{u \in U_{m}\left(\alpha_{1} \alpha_{2}\right) \backslash U_{m}} \sum_{d \bmod \alpha_{1} \alpha_{2} u c_{1} c_{2} v_{1} v_{2} \Lambda_{m}} K_{\alpha_{1} \alpha_{2}}\left(T,\left(u_{1} u_{2} c_{1} c_{2} v_{1} v_{2}, d\right) ; \widetilde{\sigma}, \widetilde{v}_{2}\right) \\
& =\sum_{\widetilde{u}_{1} \in U_{m}\left(\alpha_{1}\right) \backslash U_{m}} \sum_{d_{1}} K_{\alpha_{1}}\left(T,\left(\widetilde{u}_{1} c_{1} v_{1}, \widetilde{u}_{1} d_{1}\right) ;\right. \\
& \left.\left[\alpha_{2} \ddagger\left({ }^{t}{\widehat{\operatorname{det} c_{2}}}{\widehat{\operatorname{det} c_{2}}}^{-1}\right) \widetilde{\sigma}\right]_{\alpha_{1}}, \alpha_{2}^{-1}{\widetilde{v}_{2}}^{t}{\widehat{\operatorname{det} c_{2}}}^{-1}\right) \\
& \times \sum_{\widetilde{u}_{2} \in U_{m}\left(\alpha_{2}\right) \backslash U_{m}} \sum_{d_{2}} K_{\alpha_{2}}\left(T,\left(\widetilde{u}_{2} c_{2} v_{2}, \widetilde{u}_{2} d_{2}\right) ;\right. \\
& \left.\left[\alpha_{1} \ddagger\left({ }^{t}{\widehat{\operatorname{det} c_{1}}}{\widehat{\operatorname{det} c_{1}}}^{-1}\right) \widetilde{\sigma}\right]_{\alpha_{2}}, \alpha_{1}^{-1} \widetilde{v}_{2} t{\widehat{\operatorname{det} c_{1}}}^{-1}\right),
\end{aligned}
$$

using (2) in Lemma 11. Adding them on $v_{1} \in\left(\left(c_{1} c_{2}\right)^{-1} U_{m} c_{1} c_{2} \cap U_{m}\right) \backslash\left(c_{2}^{-1} U_{m} c_{2} \cap\right.$ $\left.U_{m}\right)$ and $v_{2} \in\left(c_{2}^{-1} U_{m} c_{2} \cap U_{m}\right) \backslash U_{m}\left(\alpha_{1}\right)$ and replacing $c_{i}$ by $C_{i}$, we get the formula in Lemma.

Lemma 24. For $\widetilde{\sigma}=\left(\begin{array}{c}* * \\ \widetilde{c} \widetilde{d}\end{array}\right) \in \Gamma_{m}, \widetilde{V}_{2} \in M_{r, m}$ and $T \in \Lambda_{n}^{\prime}$, we put

$$
S\left(T ; \widetilde{\sigma}, \widetilde{V}_{2}\right):=\sum_{c, d} \operatorname{det} c^{-k}|\operatorname{det} c|^{-2 s} K\left(T,(c, d), 1_{r} ; \widetilde{\sigma}, \widetilde{V}_{2}\right)
$$

where $(c, d)$ runs over $U_{m}(q) \backslash\left\{(c, d) \mid c, d \in M_{m}\right.$, det $\left.c \neq 0\right\} /\left(\begin{array}{cc}1_{m} & q \Lambda_{m} \\ 0 & 1_{m}\end{array}\right)$.

Then $S\left(T ; \widetilde{\sigma}, \widetilde{V}_{2}\right)$ is equal to

$$
\begin{gathered}
2 \varphi(q)^{-1} \sum_{\chi}\left\{\sum_{c_{1}} \chi\left(\operatorname{det} c_{1}\right)\left(\operatorname{det} c_{1}\right)^{-k-2 s} \sum_{d_{1}} K\left(T,\left(c_{1}, d_{1}\right)\right)\right\} \\
\times\left\{\sum_{a} \bar{\chi}(a) \sum_{c_{2}}\left(\operatorname{det} c_{2}\right)^{-k-2 s} \sum_{d_{2}} K_{q}\left(T,\left(c_{2}, d_{2}\right) ;\left(\begin{array}{c}
{ }^{t} \hat{a} \\
\hat{a}^{-1}
\end{array}\right) \widetilde{\sigma}, \widetilde{V}_{2} \hat{a}^{-1}\right)\right\}
\end{gathered}
$$

where $\varphi$ is Euler's function, $\chi$ runs over the set of Dirichlet characters defined modulo $q$ with $\chi(-1)=(-1)^{k}$, and $c_{i}, d_{i}$ run over

$$
\begin{array}{ll}
c_{1} \in U_{m} \backslash\left\{c_{1} \in M_{m} \mid \operatorname{det} c_{1}>0,\left(\operatorname{det} c_{1}, q\right)=1\right\}, & d_{1} \in M_{m} \bmod c_{1} \Lambda_{m}, \\
c_{2} \in U_{m}(q) \backslash\left\{c_{2} \in M_{m}\left|0<\operatorname{det} c_{2}\right| q^{\infty}\right\}, & d_{2} \in M_{m} \bmod q c_{2} \Lambda_{m},
\end{array}
$$

a runs over $(\mathbf{Z} / q \mathbf{Z})^{\times}$, and $\hat{a}$ stands for $\operatorname{diag}\left(1_{m-1}, a\right)$. 
Proof. By definition, $K\left(T,(c, d), 1_{r} ; \widetilde{\sigma}, \widetilde{v}_{2}\right)=K_{q}\left(T,(c, d) ; \widetilde{\sigma}, \widetilde{v}_{2}\right), \quad K(T$, $(c, d))=K_{1}\left(T,(c, d) ; \widetilde{\sigma}, \widetilde{v}_{2}\right)$. Putting $\kappa=\operatorname{diag}\left(1_{m-1},-1\right)$, we have

$$
\begin{aligned}
& S\left(T ; \widetilde{\sigma}, \widetilde{V}_{2}\right) \\
& =\sum_{\substack{c \in U_{m}(q) \backslash M_{m} \\
\operatorname{det} c>0}}(\operatorname{det} c)^{-k-2 s} \sum_{d} K_{q}\left(T,(c, d) ; \widetilde{\sigma}, \widetilde{V}_{2}\right) \\
& \quad+\sum_{\substack{c \in U_{m}(q) \backslash M_{m} \\
\operatorname{det} c>0}}(-1)^{k}(\operatorname{det} c)^{-k-2 s} \sum_{d} K_{q}\left(T,(\kappa c, \kappa d) ; \widetilde{\sigma}, \widetilde{V}_{2}\right) \\
& =\sum_{\substack{c \in U_{m}(q) \backslash M_{m} \\
\operatorname{det} c>0}}(\operatorname{det} c)^{-k-2 s} \sum_{d} K_{q}\left(T,(c, d) ; \widetilde{\sigma}, \widetilde{V}_{2}\right) \\
& \quad+(-1)^{k} \sum_{\substack{c \in U_{m}(q) \backslash M_{m} \\
\operatorname{det} c>0}}(\operatorname{det} c)^{-k-2 s} \sum_{d} K_{q}\left(T,(c, d) ;\left({ }^{t} \kappa \kappa^{-1}\right) \widetilde{\sigma}, \widetilde{V}_{2}{ }^{t} \kappa^{-1}\right)
\end{aligned}
$$

by Lemma 11. Thus putting $S^{\prime}\left(T ; \widetilde{\sigma}, \widetilde{V}_{2}\right):=\sum_{c, d}(\operatorname{det} c)^{-k-2 s} K_{q}\left(T,(c, d) ; \widetilde{\sigma}, \widetilde{V}_{2}\right)$, where $c \in U_{m}(q) \backslash\left\{c \in M_{m} \mid \operatorname{det} c>0\right\}, d \in M_{m} \bmod q c \Lambda_{m}$, we have $S\left(T ; \widetilde{\sigma}, \widetilde{V}_{2}\right)=$ $S^{\prime}\left(T ; \widetilde{\sigma}, \widetilde{V}_{2}\right)+(-1)^{k} S^{\prime}\left(T ;\left({ }^{t}{ }{ }_{\kappa^{-1}}\right) \widetilde{\sigma}, \widetilde{V}_{2}{ }^{t} \kappa^{-1}\right)$. Applying Lemma 23 to $\alpha_{1}=1$, $\alpha_{2}=q$, we have

$$
\begin{aligned}
& S^{\prime}\left(T ; \widetilde{\sigma}, \widetilde{V}_{2}\right) \\
& =\sum_{c_{1}} \sum_{c_{2}}\left(\operatorname{det}\left(c_{1} c_{2}\right)\right)^{-k-2 s} \sum_{d_{1}} K\left(T,\left(c_{1}, d_{1}\right)\right) \\
& \quad \times \sum_{d_{2}} K_{q}\left(T,\left(c_{2}, d_{2}\right) ;\left({\widehat{\operatorname{det} c_{1}}}_{\left.\left.{\widehat{\operatorname{det} c_{1}}}^{-1}\right) \tilde{\sigma}, \widetilde{V}_{2}{\widehat{\operatorname{det} c_{1}}}^{-1}\right)}\right.\right.
\end{aligned}
$$

where $c_{i}, d_{i}$ runs over the same sets as in Lemma. This is equal to

$$
\begin{aligned}
& \sum_{c_{1}} \sum_{c_{2}}\left(\operatorname{det}\left(c_{1} c_{2}\right)\right)^{-k-2 s} \sum_{d_{1}} K\left(T,\left(c_{1}, d_{1}\right)\right) \\
& \times \varphi(q)^{-1} \sum_{a} \sum_{\chi} \bar{\chi}(a) \chi\left(\operatorname{det} c_{1}\right) \sum_{d_{2}} K_{q}\left(T,\left(c_{2}, d_{2}\right) ;\left({ }_{\hat{a}^{-1}}^{\hat{a}}\right) \widetilde{\sigma}, \widetilde{V}_{2} \hat{a}^{-1}\right)
\end{aligned}
$$

where $a$ runs over $(\mathbf{Z} / q \mathbf{Z})^{\times}$and $\chi$ runs over the set of Dirichlet characters defined modulo $q$, and this is equal to

$$
\begin{aligned}
& \varphi(q)^{-1} \sum_{\chi}\left\{\sum_{c_{1}} \chi\left(\operatorname{det} c_{1}\right)\left(\operatorname{det} c_{1}\right)^{-k-2 s} \sum_{d_{1}} K\left(T,\left(c_{1}, d_{1}\right)\right)\right\} \\
& \times\left\{\sum_{a} \bar{\chi}(a) \sum_{c_{2}}\left(\operatorname{det} c_{2}\right)^{-k-2 s} \sum_{d_{2}} K_{q}\left(T,\left(c_{2}, d_{2}\right) ;\left(\hat{a}_{\hat{a}^{-1}}\right) \widetilde{\sigma}, \widetilde{V}_{2} \hat{a}^{-1}\right)\right\} .
\end{aligned}
$$


Thus we have $S\left(T ; \widetilde{\sigma}, \widetilde{V}_{2}\right)=S^{\prime}\left(T ; \widetilde{\sigma}, \widetilde{V}_{2}\right)+(-1)^{k} S^{\prime}\left(T ;\left({ }^{t}{ }{ }_{\kappa^{-1}}\right) \widetilde{\sigma}, \widetilde{V}_{2}{ }^{t} \kappa^{-1}\right)=S^{\prime}$ $\left(T ; \widetilde{\sigma}, \widetilde{V}_{2}\right)+\chi(-1)(-1)^{k} S^{\prime}\left(T ; \widetilde{\sigma}, \widetilde{V}_{2}\right)$, which completes the proof.

Lemma 25. For $T \in \Lambda_{n}^{\prime}$ and a Dirichlet character $\chi$ defined modulo $q$, we have

$$
\begin{gathered}
\sum_{c_{1} \in U_{m} \backslash\left\{c_{1} \in M_{m} \mid \begin{array}{c}
\operatorname{det} c_{1}>0, \\
\left(\operatorname{det} c_{1}, q\right)=1
\end{array}\right\}} \chi\left(\operatorname{det} c_{1}\right)\left(\operatorname{det} c_{1}\right)^{-k-2 s} \sum_{d_{1} \in M_{m} \bmod c_{1} \Lambda_{m}} K\left(T,\left(c_{1}, d_{1}\right)\right) \\
=\prod_{p \nmid q}\left\{\sum_{c \in U_{m} \backslash\left\{c \in M_{m}|0<\operatorname{det} c| p^{\infty}\right\}}\left(\chi(p) p^{-k-2 s}\right)^{\operatorname{ord}_{p}(\operatorname{det} c)} \sum_{d \in M_{m} \bmod c \Lambda_{m}} K(T,(c, d))\right\} .
\end{gathered}
$$

Proof. Applying Lemma 23 for $\alpha_{1}=\alpha_{2}=1$, we get the assertion.

Lemma 26. Let $q=\prod_{i} q_{i}$ be a decomposition such that $\left(q_{i}, q_{j}\right)=1$ for $i \neq j$ and $q_{i}$ is a power of a prime number.

We decompose a Dirichlet character $\chi$ as $\chi=\prod \chi_{i}$ where $\chi_{i}$ is a Dirichlet character defined modulo $q_{i}$. Then we have

$$
\begin{aligned}
& \sum_{a \bmod q} \bar{\chi}(a) \sum_{c_{2}}\left(\operatorname{det} c_{2}\right)^{-k-2 s} \sum_{d_{2} \in M_{m} \bmod q c_{2} \Lambda_{m}} K_{q}\left(T,\left(c_{2}, d_{2}\right) ;\left({ }^{t} \hat{a}_{\hat{a}^{-1}}\right) \tilde{\sigma} ; \widetilde{v}_{2} \hat{a}^{-1}\right) \\
& =\prod_{i}\left\{\sum_{a_{i} \bmod q_{i}} \bar{\chi}_{i}\left(a_{i}\right) \sum_{c_{i}}\left\{\prod_{j \neq i} \chi_{j}\left(\operatorname{det} c_{i}\right)\right\}\left(\operatorname{det} c_{i}\right)^{-k-2 s}\right. \\
& \left.\times \sum_{d_{i} \in M_{m}} \sum_{\bmod q_{i} c_{i} \Lambda_{m}} K_{q_{i}}\left(T,\left(c_{i}, d_{i}\right) ;\left[q q_{i}^{-1} \ddagger\left(\begin{array}{c}
{ }^{t} \hat{a}_{i} \\
\\
\\
\hat{a}_{i}^{-1}
\end{array}\right) \tilde{\sigma}\right]_{q_{i}},\left(q q_{i}^{-1}\right)^{-1} \widetilde{v}_{2} \hat{a}_{i}^{-1}\right)\right\},
\end{aligned}
$$

where $c_{2}$ in the first line runs over $U_{m}(q) \backslash\left\{c_{2} \in M_{m}\left|0<\operatorname{det} c_{2}\right| q^{\infty}\right\}$ and $c_{i}$ runs over $U_{m}\left(q_{i}\right) \backslash\left\{c_{i} \in M_{m}\left|0<\operatorname{det} c_{i}\right| q_{i}^{\infty}\right\}$.

Proof. We use induction on the number of prime factors of $q$. When $q$ is a power of a prime, the assertion is obvious. Let $\alpha_{1}, \alpha_{2}$ be relatively prime natural numbers and $\chi=\chi_{1} \chi_{2}$ a Dirichlet character with $\chi_{i}$ being defined modulo $\alpha_{i}$. Let $\xi$ be another Dirichlet character defined modulo $\alpha_{1} \alpha_{2}$ and decomposing $a \in$ $\left(\mathbf{Z} / \alpha_{1} \alpha_{2} \mathbf{Z}\right)^{\times}$as $a=a_{1} a_{2}, a_{i} \equiv 1 \bmod \alpha_{i}$, we have, by Lemma 23

$$
\begin{aligned}
& \left.\sum_{a \bmod \alpha_{1} \alpha_{2}} \bar{\chi}(a) \sum_{c \in U_{m}\left(\alpha_{1} \alpha_{2}\right) \backslash\left\{\left\{\in M_{m}|\operatorname{det} c|\left(\alpha_{1} \alpha_{2}\right)^{\infty}\right.\right.}^{\operatorname{det} c>0}\right\} \\
& \times \sum_{d \in M_{m} \bmod \alpha_{1} \alpha_{2} c \Lambda_{m}} K_{\alpha_{1} \alpha_{2}}\left(T,(c, d) ;\left({ }^{t} \hat{a}{ }_{\hat{a}^{-1}}\right) \widetilde{\sigma}, \widetilde{v}_{2} \hat{a}^{-1}\right)
\end{aligned}
$$




$$
\begin{aligned}
& =\sum_{a_{1}, a_{2}} \bar{\chi}_{1}\left(a_{2}\right) \bar{\chi}_{2}\left(a_{1}\right) \\
& \times \quad \sum \xi\left(\operatorname{det} c_{1}\right)\left(\operatorname{det} c_{1}\right)^{-k-2 s} \xi\left(\operatorname{det} c_{2}\right)\left(\operatorname{det} c_{2}\right)^{-k-2 s} \\
& c_{i} \in U_{m}\left(\alpha_{i}\right) \backslash\left\{c_{i} \in M_{m} \mid \begin{array}{c}
\operatorname{det} c_{i}>0 \\
\operatorname{det} c_{i} \mid \alpha_{i}^{\infty}
\end{array}\right\}
\end{aligned}
$$

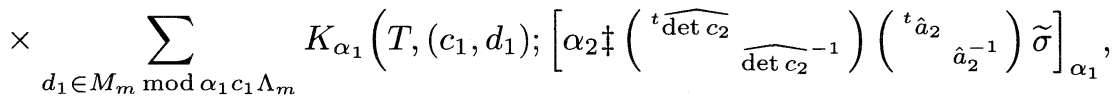

$$
\begin{aligned}
& \left.\alpha_{2}^{-1} \widetilde{v}_{2} \hat{a}_{2}^{-1}{\widehat{\operatorname{det} c_{2}}}^{-1}\right)
\end{aligned}
$$

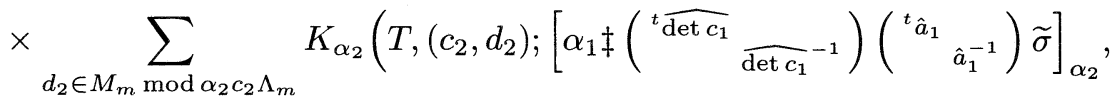

$$
\begin{aligned}
& \left.\alpha_{1}^{-1} \widetilde{v}_{2} \hat{a}_{1}^{-1}{\widehat{\operatorname{det} c_{1}}}^{-1}\right) \\
& =\left\{\sum_{a_{2} \bmod \alpha_{1}} \bar{\chi}_{1}\left(a_{2}\right) \sum_{c_{1} \in U_{m}\left(\alpha_{1}\right) \backslash\left\{\left\{\begin{array}{c}
\operatorname{det} c_{1}>0 \\
c_{1} \in M_{m}\left|\operatorname{det} c_{1}\right| \alpha_{1}^{\infty}
\end{array}\right\}\right.} \xi \chi_{2}\left(\operatorname{det} c_{1}\right)\left(\operatorname{det} c_{1}\right)^{-k-2 s}\right. \\
& \left.\times \sum_{d_{1} \in M_{m}} \sum_{\bmod \alpha_{1} c_{1} \Lambda_{m}} K_{\alpha_{1}}\left(T,\left(c_{1}, d_{1}\right) ;\left[\alpha_{2} \ddagger\left(\begin{array}{cc}
{ }^{t} \hat{a}_{2} & \\
& \hat{a}_{2}^{-1}
\end{array}\right) \widetilde{\sigma}\right]_{\alpha_{1}}, \alpha_{2}^{-1} \widetilde{v}_{2} \hat{a}_{2}^{-1}\right)\right\} \\
& \times\left\{\sum_{a_{1} \bmod \alpha_{2}} \bar{\chi}_{2}\left(a_{1}\right) \sum_{c_{2} \in U_{m}\left(\alpha_{2}\right) \backslash\left\{M_{c_{2} \mid \operatorname{det} c_{2}>0}^{\operatorname{det} c_{2} \mid \alpha_{2}^{\infty}}\right\}} \xi \chi_{1}\left(\operatorname{det} c_{2}\right)\left(\operatorname{det} c_{2}\right)^{-k-2 s}\right. \\
& \left.\times \sum_{d_{2} \in M_{m}} \sum_{\bmod \alpha_{2} c_{2} \Lambda_{m}} K_{\alpha_{2}}\left(T,\left(c_{2}, d_{2}\right) ;\left[\alpha_{1} \ddagger\left({ }^{t} a_{1}{ }_{a_{1}-1}\right) \widetilde{\sigma}\right]_{\alpha_{2}}, \alpha_{1}^{-1} \widetilde{v}_{2} \hat{a}_{1}^{-1}\right)\right\} .
\end{aligned}
$$

When $q=q_{1} q_{2}$, putting $\alpha_{i}=q_{i}$ and $\xi=1$ and exchanging $a_{1}$ and $a_{2}$, we get the assertion. Using the above equation inductively, we complete the proof.

From Lemmas 24, 25, 26 follows

Lemma 27. Let $q=\prod_{i} q_{i}$ be a decomposition such that $\left(q_{i}, q_{j}\right)=1$ for $i \neq j$ and $q_{i}$ is a power of a prime number. For $\widetilde{\sigma}=\left(\begin{array}{l}* \\ \widetilde{c} \widetilde{d}\end{array}\right) \in \Gamma_{m}, \widetilde{v}_{2} \in M_{r, m}$ and $T \in \Lambda_{n}^{\prime}$, $S\left(T ; \widetilde{\sigma}, \widetilde{v}_{2}\right)$ in Lemma 24 is equal to

$$
\begin{aligned}
& 2 \varphi(q)^{-1} \sum_{\substack{\chi \bmod q \\
\chi(-1)=(-1)^{k}}} \prod_{p \dagger q}\left\{\sum_{c \in U_{m} \backslash\left\{c \in M_{m}|0<\operatorname{det} c| p^{\infty}\right\}}\left(\chi(p) p^{-k-2 s}\right)^{\operatorname{ord}_{p} \operatorname{det} c}\right. \\
& \left.\times \sum_{d \bmod c \Lambda_{m}} K(T,(c, d))\right\} \prod_{i}\left\{\sum_{a_{i} \bmod q_{i}} \bar{\chi}_{i}\left(a_{i}\right)\right.
\end{aligned}
$$




$$
\begin{aligned}
& \times \sum_{c_{i} \in U_{m}\left(q_{i}\right) \backslash\left\{c_{i} \in M_{m} \mid \begin{array}{c}
\operatorname{det} c_{i}>0 \\
\left.\operatorname{det} c_{i} \mid q_{i}^{\infty}\right\}
\end{array}\right.}\left\{\prod_{j \neq i} \chi_{j}\left(\operatorname{det} c_{i}\right)\right\}\left(\operatorname{det} c_{i}\right)^{-k-2 s} \\
& \left.\times \sum_{d_{i} \bmod q_{i} c_{i} \Lambda_{m}} K_{q_{i}}\left(T,\left(c_{i}, d_{i}\right) ;\left[q q_{i}^{-1} \ddagger\left(\begin{array}{c}
{ }^{t} \hat{a}_{i} \\
\hat{a}_{i}^{-1}
\end{array}\right) \widetilde{\sigma}\right]_{q_{i}},\left(q q_{i}^{-1}\right)^{-1} \widetilde{v}_{2} \hat{a}_{i}^{-1}\right)\right\},
\end{aligned}
$$

where $\chi=\prod_{i} \chi_{i}$ and $\chi_{i}$ is a Dirichlet character defined modulo $q_{i}$.

Let us study factors in the above infinite product for $p \nmid q$. To evaluate the power series

$$
\sum_{c \in U_{n} \backslash\left\{c \in M_{n} \mid \begin{array}{c}
\operatorname{det} c>0 \\
\operatorname{det} c \mid p^{\infty}
\end{array}\right\}} x^{\operatorname{ord}_{p} \operatorname{det} c} \sum_{d \in M_{n} \bmod c \Lambda_{n}} K(T,(c, d))
$$

for $T \in \Lambda_{n}^{\prime}$ with $\operatorname{det} T_{1}^{(r)} \neq 0$, we consider

$$
\beta_{p}(T, x):=\sum_{c, d} x^{\operatorname{ord}_{p} \operatorname{det} c} e\left(\operatorname{tr} T c^{-1} d\right)
$$

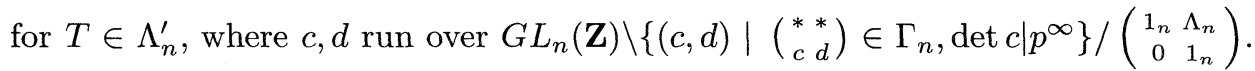
If $T \notin \Lambda_{n}^{\prime}$, then we put $\beta_{p}(T, x)=0$. It is known [K2] that if $\operatorname{det} T \neq 0$, then

$$
\begin{aligned}
& \beta_{p}\left(T, p^{-k}\right) \\
& =\lim _{t \rightarrow \infty}\left(p^{t}\right)^{(n+1) n / 2-2 n k} \sharp\left\{X \in M_{2 k, n}\left(\mathbf{Z}_{p} / p^{t} \mathbf{Z}_{p}\right) \mid H_{k}[X] \equiv T \bmod p^{t} \Lambda_{n}^{\prime}\left(\mathbf{Z}_{p}\right)\right\},
\end{aligned}
$$

where $H_{k}:=\frac{1}{2}\left(\begin{array}{cc}0 & 1_{k} \\ 1_{k} & 0\end{array}\right)$ and $\Lambda_{n}^{\prime}\left(\mathbf{Z}_{p}\right):=\Lambda_{n}^{\prime} \otimes \mathbf{z} \mathbf{Z}_{p}$ if $2 k \geq n$, and

$$
\beta_{p}(T, x)=\sum_{G}\left(p^{n+1} x^{2}\right)^{\operatorname{ord}_{p} \operatorname{det} G} \gamma_{p}\left(T\left[G^{-1}\right], x\right),
$$

where $G$ runs over $G L_{n}\left(\mathbf{Z}_{p}\right) \backslash\left(M_{n}\left(\mathbf{Z}_{p}\right) \cap G L_{n}\left(\mathbf{Q}_{p}\right)\right)$ and $\gamma_{p}(T, x):=0$ if $T \notin \Lambda_{n}^{\prime}\left(\mathbf{Z}_{p}\right)$, otherwise we put

$$
\gamma_{p}(T, x):=(1-x)\left(1+\epsilon p^{n-d / 2} x\right) \prod_{1 \leq i<n-d / 2}\left(1-p^{2 i} x^{2}\right)
$$

where $d, \epsilon$ are defined as follows:

Let $V$ be a quadratic space over $\mathbf{Z} / p \mathbf{Z}$ with basis $\left\{v_{i}\right\}_{i=1}^{n}$ such that the quadratic form $q$ is given by

$$
q\left(\sum x_{i} v_{i}\right):=T\left[\begin{array}{c}
x_{1} \\
\vdots \\
x_{n}
\end{array}\right] \bmod p
$$


and the associated bilinear form $b$ is defined by $b(x, y):=q(x+y)-q(x)-q(y)$. Then $n-d$ is the dimension of the $\operatorname{radical} \operatorname{Rad}(V):=\{v \in V \mid q(v)=0, b(v, V)=0\}$. If $d$ is odd, then we put $\epsilon=0$. Suppose that $d$ is even and put $V=V_{0} \perp \operatorname{Rad}(V)$. If $V_{0}$ is hyperbolic, that is $V_{0}=0$ or $V_{0}$ is isometric to the quadratic space corresponding to $H_{k}$ for some $k$, then we put $\epsilon=1$, otherwise $\epsilon=-1$.

Lemma 28. For $T \in \Lambda_{n}^{\prime}$ with $\operatorname{det} T_{1}^{(r)} \neq 0$, we have

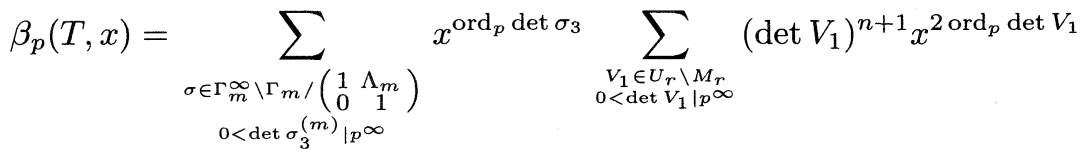

$$
\begin{aligned}
& \times \gamma_{p}\left(T_{1}\left[V_{1}^{-1}\right], x\right) K\left(T\left[\left(\begin{array}{ll}
V_{1} & \\
& 1_{m}
\end{array}\right)^{-1}\right],\left(\sigma_{3}, \sigma_{4}\right)\right) .
\end{aligned}
$$

Proof. From Lemma 6,7 follows that a complete set of representatives of $\Gamma_{n}^{\infty} \backslash\left\{K \in \Gamma_{n}\left|\operatorname{det} K_{3}^{(n)}\right| p^{\infty}\right\} /\left(\begin{array}{cc}1 & \Lambda_{n} \\ 0 & 1\end{array}\right)$ is given by $M \uparrow N(v, \sigma)$, where $\sigma \in$ $\Gamma_{m}^{\infty} \backslash \Gamma_{m} /\left(\begin{array}{cc}1 & \Lambda_{m} \\ 0 & 1\end{array}\right)$ with $0<\operatorname{det} \sigma_{3}^{(m)} \mid p^{\infty}, v \in{ }^{t} P_{n, r} \backslash U_{n} /\left(\begin{array}{cc}1_{r} & M_{r, m}{ }^{t} \sigma_{3} \\ 0 & 1_{m}\end{array}\right)$ with $0<$ $\operatorname{det} v_{1}^{(r)} \mid p^{\infty}, M \in \Gamma_{r}^{\infty} \backslash \Gamma_{r} /\left(\begin{array}{cc}1_{r} & \Lambda_{r}\left[{ }^{t} v_{1}\right] \\ 0 & 1_{r}\end{array}\right)$ with $0<\operatorname{det} M_{3}^{(r)} \mid p^{\infty}$. Lemma 6 implies

$$
\begin{aligned}
& \beta_{p}(T, x) \\
& \quad=\sum_{\sigma, v, M} x^{\operatorname{ord}\left(\operatorname{det} M_{3} \operatorname{det} \sigma_{3} \operatorname{det} v_{1}^{2}\right)} e\left(\operatorname{tr} T\left(\begin{array}{cc}
M_{3}^{-1} M_{4}\left[{ }^{t} v_{1}^{-1}\right]+\sigma_{1} \sigma_{3}^{-1}\left[{ }^{t}\left(v_{1}^{-1} v_{2}\right)\right] & * \\
-\sigma_{3}^{-1}\left(v_{1}^{-1} v_{2}\right) & \sigma_{3}^{-1} \sigma_{4}
\end{array}\right)\right)
\end{aligned}
$$

where $\sigma, v, M$ run over above sets, and this is equal to

$$
\sum_{\sigma, v}\left(\operatorname{det} v_{1}\right)^{r+1} \beta_{p}\left(T_{1}\left[v_{1}^{-1}\right], x\right) x^{\operatorname{ord}_{p}\left(\operatorname{det} \sigma_{3} \operatorname{det} v_{1}^{2}\right)} e\left(\operatorname{tr} T\left(\begin{array}{cc}
\sigma_{1} \sigma_{3}^{-1}\left[{ }^{t}\left(v_{1}^{-1} v_{2}\right)\right] & * \\
-\sigma_{3}^{-1 t}\left(v_{1}^{-1} v_{2}\right) & \sigma_{3}^{-1} \sigma_{4}
\end{array}\right)\right)
$$

since $\left[\Lambda_{r}: \Lambda_{r}\left[{ }^{t} v_{1}\right]\right]=\operatorname{det} v_{1}^{r+1}$

$$
\begin{aligned}
= & \sum_{\sigma, v} \operatorname{det} v_{1}^{r+1}\left\{\sum_{g}\left(p^{r+1} x^{2}\right)^{\operatorname{ord}_{p} \operatorname{det} g} \gamma_{p}\left(T_{1}\left[v_{1}^{-1} g^{-1}\right], x\right)\right\} x^{\operatorname{ord}_{p}\left(\operatorname{det} \sigma_{3} \operatorname{det} v_{1}^{2}\right)} \\
& \times e\left(\operatorname{tr} T\left(\begin{array}{cc}
\sigma_{1} \sigma_{3}^{-1}\left[{ }^{t}\left(v_{1}^{-1} v_{2}\right)\right] & * \\
-\sigma_{3}^{-1 t}\left(v_{1}^{-1} v_{2}\right) & \sigma_{3}^{-1} \sigma_{4}
\end{array}\right)\right)
\end{aligned}
$$

where $g$ runs over $U_{r} \backslash\left\{g \in M_{r}|0<\operatorname{det} g| p^{\infty}\right\}$. The mapping $v \mapsto\left(v_{1}^{(r)}, v_{2}\right)$ gives a bijection from $\left\{v \in{ }^{t} P_{n, r} \backslash U_{n} /\left(\begin{array}{cc}1_{r} & M_{r, m}{ }^{t} \sigma_{3} \\ 0 & 1_{m}\end{array}\right)\left|0<\operatorname{det} v_{1}\right| p^{\infty}\right\}$ to $\widetilde{V}_{p r}:=$ $U_{r} \backslash\left\{\left(v_{1}, v_{2}\right) \in M_{r} \times M_{r, m}\left|0<\operatorname{det} v_{1}\right| p^{\infty},\left(v_{1}, v_{2}\right): \operatorname{primitive~}, v_{2} \bmod v_{1} M_{r, m}{ }^{t} \sigma_{3}\right\}$ and the mapping $g,\left(v_{1}, v_{2}\right) \mapsto\left(g v_{1}, g v_{2}\right)$ gives also a bijection from $\sqcup_{\left(v_{1}, v_{2}\right) \in \widetilde{V}_{p r}} U_{r} \backslash$ $\left\{g \in M_{r}|0<\operatorname{det} g| p^{\infty}\right\} \times\left(v_{1}, v_{2}\right)$ to $\widetilde{V}:=U_{r} \backslash\left\{\left(V_{1}, V_{2}\right) \in M_{r} \times M_{r, m} \mid 0<\right.$ 
$\left.\operatorname{det} V_{1} \mid p^{\infty}, V_{2} \bmod V_{1} M_{r, m}{ }^{t} \sigma_{3}\right\}$. The first one is easy. To see the second bijectivity, suppose that $\left(v_{1}, v_{2}\right),\left(v_{1}^{\prime}, v_{2}^{\prime}\right) \in \widetilde{V}_{p r}, g, g^{\prime} \in M_{r}$ with $0<\operatorname{det} g, \operatorname{det} g^{\prime}$ and $\operatorname{det} g g^{\prime} \mid p^{\infty}$ are given and $\left(g^{\prime} v_{1}^{\prime}, g^{\prime} v_{2}^{\prime}\right)=u\left(g v_{1}, g v_{2}\right)\left(\begin{array}{cc}1_{r} & x \\ 0 & 1_{m}\end{array}\right)$ for $u \in U_{r}, x \in M_{r, m}{ }^{t} \sigma_{3}$. Then the primitivity of $\left(v_{1}, v_{2}\right),\left(v_{1}^{\prime}, v_{2}^{\prime}\right)$ implies $(u g)^{-1} g^{\prime} \in G L_{r}(\mathbf{Z})$, and so $(u g)^{-1} g^{\prime} \in U_{r}$ which means $g^{\prime} \in U_{r} g$, and it yields that $\left(v_{1}, v_{2}\right)$ and $\left(v_{1}^{\prime}, v_{2}^{\prime}\right)$ are the same in $\widetilde{V}_{p r}$. Thus the injectivity has been proved. Elementary divisor theorem yields the surjectivity. Putting $\left(V_{1}, V_{2}\right):=\left(g v_{1}, g v_{2}\right)$ and continuing the calculation, we have

$$
\begin{aligned}
& \beta_{p}(T, x)=\sum_{\sigma} \sum_{\left(V_{1}, V_{2}\right) \in \tilde{V}}\left(\operatorname{det} V_{1}\right)^{r+1} x^{\operatorname{ord}_{p}\left(\operatorname{det} \sigma_{3}\right)+2 \operatorname{ord}_{p} \operatorname{det} V_{1}} \\
& \quad \times \gamma_{p}\left(T_{1}\left[V_{1}^{-1}\right], x\right) e\left(\operatorname{tr} T\left(\begin{array}{c}
\sigma_{1} \sigma_{3}^{-1}\left[\left[^{t}\left(V_{1}^{-1} V_{2}\right)\right]\right. \\
-\sigma_{3}^{-1 t}\left(V_{1}^{-1} V_{2}\right) \sigma_{3}^{-1} \sigma_{4}
\end{array}\right)\right) \\
& =\sum_{\sigma} \sum_{V_{1} \in U_{r} \backslash\left\{V_{1} \in M_{r}\left|0<\operatorname{det} V_{1}\right| p^{\infty}\right\}}\left(\operatorname{det} V_{1}\right)^{r+1} x^{\operatorname{ord}_{p}\left(\operatorname{det} \sigma_{3}\right)+2 \operatorname{ord}_{p} \operatorname{det} V_{1}}
\end{aligned}
$$

(1)

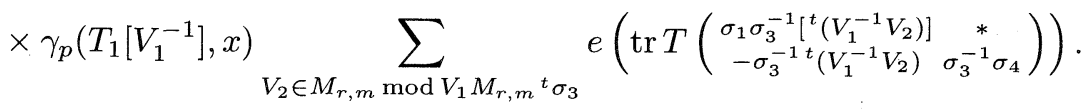

The partial sum on $V_{2}$ is, by writing $V_{2}=x+y^{t} \sigma_{3}\left(x \in M_{r, m} \bmod M_{r, m}{ }^{t} \sigma_{3}, y \in\right.$ $\left.M_{r, m} \bmod V_{1} M_{r, m}\right)$,

$$
\begin{aligned}
& \sum_{x, y} e\left(\operatorname{tr} T\left(\begin{array}{cc}
\sigma_{1} \sigma_{3}^{-1}\left[{ }^{t}\left(V_{1}^{-1}\left(x+y^{t} \sigma_{3}\right)\right)\right] & * \\
-\sigma_{3}^{-1 t}\left(V_{1}^{-1}\left(x+y^{t} \sigma_{3}\right)\right) & \sigma_{3}^{-1} \sigma_{4}
\end{array}\right)\right)
\end{aligned}
$$

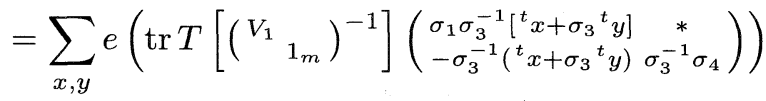

$$
\begin{aligned}
& =\sum_{x} e\left(\operatorname{tr} T\left[\left(\begin{array}{ll}
V_{1} & \\
& 1_{m}
\end{array}\right)^{-1}\right]\left(\begin{array}{cc}
\sigma_{1} \sigma_{3}^{-1}\left[{ }^{t} x\right] & * \\
-\sigma_{3}^{-1 t} x & \sigma_{3}^{-1} \sigma_{4}
\end{array}\right)\right) \\
& \times \sum_{y} e\left(\operatorname{tr} T\left[\left(\begin{array}{ll}
V_{1} & \\
& 1_{m}
\end{array}\right)^{-1}\right]\left(\begin{array}{cc}
x \sigma_{1}{ }^{t} y+y^{t} \sigma_{1}{ }^{t} x+{ }^{t} \sigma_{3} \sigma_{1}[y] & * \\
-{ }^{t} y & 0
\end{array}\right)\right)
\end{aligned}
$$

here by vitue of the existence of $\gamma_{p}\left(T_{1}\left[V_{1}^{-1}\right], x\right)$ at (1), we may assume $T_{1}\left[V_{1}^{-1}\right] \in \Lambda_{r}^{\prime}$ and so the above expression is equal to

$$
\sum_{x} e\left(\operatorname{tr} T\left[\left(\begin{array}{ll}
V_{1} & \\
& 1_{m}
\end{array}\right)^{-1}\right]\left(\begin{array}{cl}
\sigma_{1} \sigma_{3}^{-1}\left[{ }^{t} x\right] & * \\
-\sigma_{3}^{-1 t} x & \sigma_{3}^{-1} \sigma_{4}
\end{array}\right) \times \begin{cases}\operatorname{det} V_{1}^{m} & \text { if } 2^{t} V_{1}^{-1} T_{2} \in M_{r, m} \\
0 & \text { otherwise }\end{cases}\right.
$$

The conditions $T_{1}\left[V_{1}^{-1}\right] \in \Lambda_{r}^{\prime}$ and $2{ }^{t} V_{1}^{-1} T_{2} \in M_{r, m}$ are equivalent to $T\left[\left(\begin{array}{ll}V_{1} & \\ & 1_{m}\end{array}\right)^{-1}\right]$ $\in \Lambda_{n}^{\prime}$ and then the sum on $x$ becomes $K\left(T\left[\left(\begin{array}{cc}V_{1} & \\ & 1_{m}\end{array}\right)^{-1}\right],\left(\sigma_{3}, \sigma_{4}\right)\right)$. Thus, we get the desired result. 
Lemma 29. For $T \in \Lambda_{n}^{\prime}$ with $\operatorname{det} T_{1}^{(r)} \neq 0$, we have

$$
\begin{gathered}
\sum_{c \in U_{m} \backslash\left\{c \in M_{m}|0<\operatorname{det} c| p^{\infty}\right\}} x^{\operatorname{ord} \operatorname{det} c} \sum_{d \in M_{m} \bmod c \Lambda_{m}} K(T,(c, d)) \\
=\gamma_{p}\left(T_{1}, x\right)^{-1} \sum_{\substack{V_{1} \in U_{r} \backslash M_{r} \\
0<\operatorname{det} V_{1} \mid p^{\infty}}} \mu\left(\mathbf{Z}^{r} / \mathbf{Z}^{r} V_{1}\right) \operatorname{det} V_{1}^{n+1} x^{2 \operatorname{ord} \operatorname{det} V_{1}} \beta_{p}\left(T\left[\left(\begin{array}{ll}
V_{1} & 1_{m}
\end{array}\right)^{-1}\right], x\right) .
\end{gathered}
$$

Proof. Consider a vector space $V=\mathbf{Q}\left[v_{1}, \cdots, v_{n}\right]$ and introduce a bilinear form $B$ on it by $\left(B\left(v_{i}, v_{j}\right)\right)=T$. Put $L_{0}:=\mathbf{Z}\left[v_{1}, \cdots, v_{r}\right], L_{1}:=\mathbf{Z}\left[v_{r+1}, \cdots, v_{n}\right]$ and $L:=L_{0} \oplus L_{1}$. Now we choose a lattice $\widetilde{L} \supset L_{0}$ so that $\left[\widetilde{L}: L_{0}\right] \mid p^{\infty}$ and if a lattice $M$ on $\mathbf{Q} L_{0}$ satisfies $M \supset L_{0},\left[M: L_{0}\right] \mid p^{\infty}$ and $B(x, x) \in \mathbf{Z}$ for every $x \in M \oplus L_{1}$, then $M \subset \widetilde{L}$. Such a lattice $\widetilde{L}$ exists since $\operatorname{det} T_{1} \neq 0$. For a lattice $N=\mathbf{Z}\left[u_{1}, \cdots, u_{r}\right]$ on $\mathbf{Q} L_{0}$, we put

$$
\begin{gathered}
\gamma_{p}(N, x):=\gamma_{p}\left(\left(\left(B\left(u_{i}, u_{j}\right)\right)_{1 \leq i, j \leq r}, x\right),\right. \\
K\left(N \oplus L_{1},(c, d)\right):=K\left(\left(\left(B\left(u_{i}, u_{j}\right)\right)_{1 \leq i, j \leq n},(c, d)\right)\right.
\end{gathered}
$$

where $u_{i}:=v_{i}$ if $i>r$. If $\left(B\left(u_{i}, u_{j}\right)\right) \notin \Lambda_{r}^{\prime}$ or $\Lambda_{n}^{\prime}$, then $\gamma_{p}(N, x)$ or $K(N \oplus$ $\left.L_{1},(c, d)\right)=0$ by definitions. Since $\gamma_{p}(N, x)$ is independent of a choice of bases of $N, \gamma_{p}(N, x)$ is well-defined. Suppose $\left(u_{1}, \cdots, u_{r}\right) w\left(w \in G L_{r}(\mathbf{Z})\right)$ be another basis of $N$. Then the corresponding matrix is $\left(B\left(u_{i}, u_{j}\right)\right)\left[\left(\begin{array}{cc}w & \\ 1_{m}\end{array}\right)\right]$ and then (3) in Lemma 11 yields $K\left(\left(\left(B\left(u_{i}, u_{j}\right)\right)\left[\left(\begin{array}{cc}w & \\ & 1_{m}\end{array}\right)\right],(c, d)\right)=K\left(\left(\left(B\left(u_{i}, u_{j}\right)\right),(c, d)\right)\right.\right.$. Thus $K\left(N \oplus L_{1},(c, d)\right)$ is well-defined. For a lattice $N$ on $\mathbf{Q} L_{0}$, we put

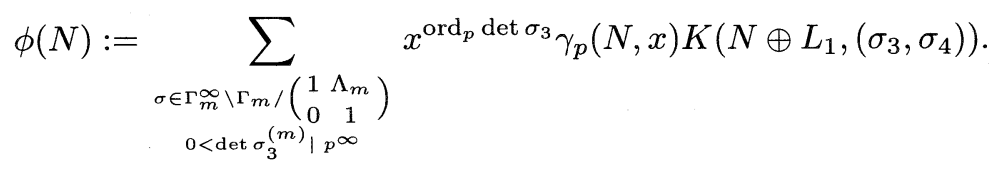

Then the previous lemma yields for $\widetilde{L} \supset M \supset L_{0}$

$$
\beta_{p}\left(M \oplus L_{1}, x\right):=\beta_{p}(\widetilde{T}, x)=\sum_{\widetilde{L} \supset N \supset M}[N: M]^{n+1} x^{2 \operatorname{ord}_{p}[N: M]} \phi(N),
$$

where $\widetilde{T}=\left(B\left(y_{i}, y_{j}\right)\right)$ for a basis $\left\{y_{i}\right\}$ of $M \oplus L_{1}$. It means

$$
\left[M: L_{0}\right]^{n+1} x^{2 \operatorname{ord}_{p}\left[M: L_{0}\right]} \beta_{p}\left(M \oplus L_{1}, x\right)=\sum_{\tilde{L} \supset N \supset M}\left[N: L_{0}\right]^{n+1} x^{2 \operatorname{ord}_{p}\left[N: L_{0}\right]} \phi(N) .
$$

Applying Lemma 8 to $\widetilde{L} / L_{0}$, we have

$$
\phi\left(L_{0}\right)=\sum_{\widetilde{L} \supset N \supset L_{0}} \mu\left(N / L_{0}\right)\left[N: L_{0}\right]^{n+1} x^{2 \operatorname{ord}_{p}\left[N: L_{0}\right]} \beta_{p}\left(N \oplus L_{1}, x\right),
$$


which is our assertion.

Lemmas 27 and 29 imply

Lemma 30. For $T \in \Lambda_{n}^{\prime}$ with $\operatorname{det} T_{1}^{(r)} \neq 0, \widetilde{\sigma} \in \Gamma_{m}, \widetilde{v}_{2} \in M_{r, m}, S\left(T ; \widetilde{\sigma}, \widetilde{v}_{2}\right)$ defined in Lemma 24 is equal to

$$
\begin{aligned}
& 2 \varphi(q)^{-1} \sum_{\substack{\chi \bmod q \\
\chi(-1)=(-1)^{k}}}\left\{\prod_{p \dagger q} \gamma_{p}\left(T_{1}, \chi(p) p^{-k-2 s}\right)^{-1}\right\}\left\{\sum_{\substack{V_{1} \in U_{r} \backslash M_{r} \\
\operatorname{det} V_{1}>0,\left(\operatorname{det} V_{1}, q\right)=1}} \mu\left(\mathbf{Z}^{r} / \mathbf{Z}^{r} V_{1}\right)\right. \\
& \left.\times \chi\left(\operatorname{det} V_{1}\right)^{2}\left(\operatorname{det} V_{1}\right)^{n+1-2 k-4 s} \prod_{p \nmid q} \beta_{p}\left(T\left[\left(\begin{array}{ll}
V_{1} & 1_{m}
\end{array}\right)^{-1}\right], \chi(p) p^{-k-2 s}\right)\right\} \\
& \times \prod_{i}\left\{\sum_{a_{i} \bmod q_{i}} \bar{\chi}_{i}\left(a_{i}\right) \sum_{c_{i}}\left\{\prod_{j \neq i} \chi_{j}\left(\operatorname{det} c_{i}\right)\right\}\left(\operatorname{det} c_{i}\right)^{-k-2 s}\right. \\
& \left.\times \sum_{d_{i}} K_{q_{i}}\left(T,\left(c_{i}, d_{i}\right) ;\left[q q_{i}^{-1} \ddagger\left(\begin{array}{c}
{ }^{t} \hat{a}_{i} \\
\hat{a}_{i}^{-1}
\end{array}\right) \widetilde{\sigma}\right]_{q_{i}},\left(q q_{i}^{-1}\right)^{-1} \widetilde{v}_{2} \hat{a}_{i}^{-1}\right)\right\},
\end{aligned}
$$

where $c_{i}$ runs over $U_{m}\left(q_{i}\right) \backslash\left\{c_{i} \in M_{m}\left|0<\operatorname{det} c_{i}\right| q_{i}^{\infty}\right\}$ and $d_{i}$ runs over $M_{m} \bmod$ $q_{i} c_{i} \Lambda_{m}$.

REMARK. The final sum on $c_{i}, d_{i}$ seems to be a finite sum if $\operatorname{det} T \neq 0$. It is true if $m=r=1, n=2$. I do not know whether it is true or not for $n>2$, nor the meaning of the sums.

Now we have from Lemmas 18,30

THeOREM 1 Let $f(z)$ be a cusp form of degree $r$, weight $k$ and level $q$, and let $M \in \Gamma_{n}$.

$$
\begin{aligned}
& \text { For } \eta=\left(\begin{array}{cccc}
a_{1}^{(r)} & 0 & & b_{1}^{(r)} * \\
* & * & & * \\
c_{1}^{(r)} & 0 & d_{1}^{(r)} & * \\
0 & 0 & 0 & d_{4}^{(m)}
\end{array}\right) \in \Delta_{n, r} \text {, we put } \\
& \left(\operatorname{det} d_{4}\right)^{k} f \mid\left(\begin{array}{ll}
a_{1} & b_{1} \\
c_{1} & d_{1}
\end{array}\right)(\tau)=\sum_{t>0} b(t ; \eta) e(\operatorname{tr} t \tau / q) \quad\left(\tau \in H_{r}\right) .
\end{aligned}
$$

Then putting for $T \in \Lambda_{n}^{\prime}$

$$
\mathcal{F}(T, y ; f, M):=q^{-r(r+1) / 2} \int_{x \in \Lambda_{n}(\mathbf{R}) / q \Lambda_{n}} \sum_{K}(f \| K)(z) e(-\operatorname{tr} T x / q) d x,
$$

where $K$ runs over

$$
\Delta_{n, r} \backslash\left\{K \in \Gamma_{n} \mid \operatorname{rank}\left(0,1_{m}\right) K_{3}^{(n)}=m, \sigma \equiv M \bmod q\right\},
$$


we have

$$
\begin{aligned}
& 2^{-1} \varphi(q) \mathcal{F}(T, y ; f, M) \\
& =\left[\Gamma_{m}^{\infty}: \Gamma_{m}^{\infty}(q)\right]^{-1}\left[P_{n, r}: P_{n, r}(q)\right]^{-2}\left[G L_{r}(\mathbf{Z}): U_{r}(q)\right]^{-1} \\
& \times \sum_{w \in U_{n} / P_{n, r}(q)} H\left(q^{-1} T[w], y\left[{ }^{t} w^{-1}\right] ; s\right)
\end{aligned}
$$

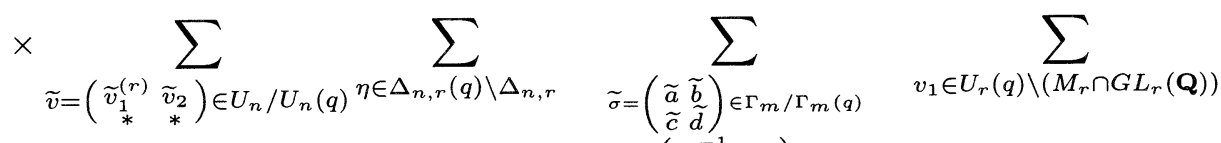

$$
\begin{aligned}
& \eta N(\tilde{v}, \tilde{\sigma})\left(w^{-1}{ }^{t} w\right) \equiv M \bmod q \\
& \times\left|\operatorname{det} v_{1}\right|^{m-2 s}\left\{\sum_{\substack{g \in U_{r}(q) \backslash\left(\mathcal{M}_{r} \cap G L_{r}(\mathbf{Q})\right) \\
g v_{1} \equiv \widetilde{v}_{1} \bmod q}} \mu\left(\mathbf{Z}^{r} / \mathbf{Z}^{r} g\right)|\operatorname{det} g|^{-2 s} b\left(T[w]_{1}^{(r)}\left[v_{1}^{-1} g^{-1}\right] ; \eta\right)\right\} \\
& \times \sum_{\substack{\chi \bmod q \\
\chi(-1)=(-1)^{k}}}\left\{\prod_{p \nmid q} \gamma_{p}\left(T[w]_{1}\left[v_{1}^{-1}\right], \chi(p) p^{-k-2 s}\right)^{-1}\right\} \\
& \times\left\{\sum_{\substack{V_{1} \in U_{r} \backslash M_{r} \\
\operatorname{det} V_{1}>0,\left(\operatorname{det} V_{1}, q\right)=1}} \mu\left(\mathbf{Z}^{r} / \mathbf{Z}^{r} V_{1}\right) \chi\left(\operatorname{det} V_{1}\right)^{2}\right. \\
& \left.\times\left(\operatorname{det} V_{1}\right)^{n+1-2 k-4 s} \prod_{p \nmid q} \beta_{p}\left(T\left[w\left(V_{V_{1} v_{1}}{ }^{1_{m}}\right)^{-1}\right], \chi(p) p^{-k-2 s}\right)\right\} \\
& \times \sum_{\substack{\widetilde{V}_{2} \in M_{r, m}, m \bmod q M_{r, m} \\
g \widetilde{V}_{2} \equiv \widetilde{v}_{2} \bmod q}} \prod_{i}\left\{\sum_{a_{i} \bmod q_{i}} \bar{\chi}_{i}\left(a_{i}\right)\right. \\
& \times \sum_{c_{i} \in U_{m}\left(q_{i}\right) \backslash\left\{c_{i} \in M_{m}\left|0<\operatorname{det} c_{i}\right| q_{i}^{\infty}\right\}}\left\{\prod_{j \neq i} \chi_{j}\left(\operatorname{det} c_{i}\right)\right\}\left(\operatorname{det} c_{i}\right)^{-k-2 s} \\
& \times \sum_{d_{i} \in M_{m} \bmod q_{i} c_{i} \Lambda_{m}} K_{q_{i}}\left(T\left[w\left({ }^{v_{1}} 1_{m}\right)^{-1}\right],\left(c_{i}, d_{i}\right) ;\right.
\end{aligned}
$$

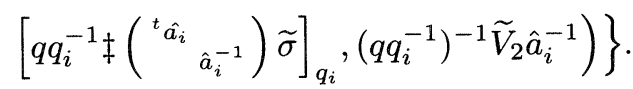

Here $q=\prod_{i} q_{i}$ is a decomposition such that $\left(q_{i}, q_{j}\right)=1$ for $i \neq j$ and $q_{i}$ is a power of a prime number, $\chi_{i}$ is a Dirichlet character defined modulo $q_{i}$ such that $\chi=\prod \chi_{i}, K_{q_{i}}$ is defined at the beginning of this section, $\hat{a}:=\operatorname{diag}\left(1_{m-1}, a\right)$ and $[\alpha \ddagger \sigma]_{\beta}$ stands for an element in $\Gamma_{m}$ which is congruent modulo $\beta$ to $\left(\begin{array}{cc}\alpha a & b \\ c & \alpha^{-1} d\end{array}\right)$ for $\alpha$ where $\sigma=\left(\begin{array}{ll}a & b \\ c & d\end{array}\right)$.

REMARK. Since $T[w]_{1}^{(r)}$ is the Bigl upper $r \times r$ submatrix of $T[w]$ by 
definition, $b\left(T[w]_{1}\left[v_{1}^{-1} g^{-1}\right] ; \eta\right)=0$ unless $0<T[w]_{1}\left[v_{1}^{-1} g^{-1}\right] \in \Lambda_{r}^{\prime}$. Also $\beta_{p}\left(T\left[w\left(\begin{array}{ll}V_{1} v_{1} & \\ & 1_{m}\end{array}\right)^{-1}\right], \chi(p) p^{-k-2 s}\right)=0$ unless $T\left[w\left(\begin{array}{cc}V_{1} v_{1} & \\ & 1_{m}\end{array}\right)^{-1}\right] \in \Lambda_{n}^{\prime}$. Therefore the sum on $g, v_{1}$ and $V_{1}$ is a finite sum, and $\mathcal{F}(T, y ; f, M)=0$ if $\operatorname{rank} T<r$.

TheOREm 2. Let $f$ be the cusp form in Theorem 1, and put

$$
E_{n, r}^{k}(f, s ; q, M)(z):=\sum_{K}\left(f \|_{s} K\right)(z) \quad \text { for } z \in H_{n}
$$

where $K$ runs over $\Delta_{n, r}(q) \backslash\left\{K \in \Gamma_{n} \mid K \equiv M \bmod q\right\}$. The Fourier coefficient at $0<T \in \Lambda_{n}^{\prime}$ of $E_{n, r}^{k}(f, s ; q, M)$ is given by $\mathcal{F}(T, y ; f, M)$ in Theorem 1.

Proof. A complete set of representatives of $\Delta_{n, r} \backslash \Gamma_{n}$ is given by

$$
\sqcup_{\mu=r}^{n} L_{n}^{r, \mu},
$$

where $L_{n}^{r, \mu}:=\left\{\sigma \uparrow\left({ }^{t}{ }_{{ }_{u^{-1}}}\right) \in \Gamma_{n} \mid \sigma \in \Delta_{\mu, r} \backslash \Gamma_{\mu}, \operatorname{rank}\left(0,1_{\mu-r}\right) \sigma_{3}^{(\mu)}=\mu-r, u \in\right.$ $\left.U_{n} / P_{n, \mu}\right\}$. The proof proceeds as follows:

If, for $M=\left(\begin{array}{ll}A & B \\ C & D\end{array}\right) \in \Gamma_{n}, \operatorname{rank}\left(0,1_{m}\right) C=\mu-r(r \leq \mu \leq n)$, then there exist $g \in G L_{m}(\mathbf{Z})$ and $v \in G L_{n}(\mathbf{Z})$ such that $g\left(\left(0,1_{m}\right) C\right)^{t} v^{-1}=\left(\underset{0^{(n-\mu, n)}}{*}\right)$. Then taking

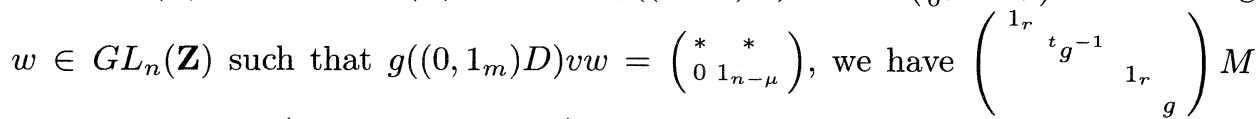
$\left(\begin{array}{ll}{ }^{t}(v w)^{-1} & \\ & v w\end{array}\right)=\left(\begin{array}{c}C_{1}^{(\mu, n)} \\ 0^{(n-\mu, n)}\end{array}\left(\begin{array}{cc}* & * \\ 0 & { }^{*} \\ 0 & 1_{n-\mu}\end{array}\right)\right) \in \Delta_{n, \mu} \subset \Delta_{n, r} \times \Gamma_{\mu} \uparrow$. Thus $M \in \Delta_{n, r} \Gamma_{\mu} \uparrow$ $\times\left\{\left(\begin{array}{cc}{ }^{t} u & \\ & u^{-1}\end{array}\right) \mid u \in G L_{n}(\mathbf{Z})\right\}$. By the assumption, $\operatorname{rank}\left(0,1_{m}\right)\left(\begin{array}{c}1_{r} \\ g^{-1}\end{array}\right)\left(\begin{array}{c}C_{1} \\ 0\end{array}\right)=\mu-r$ holds and hence $\operatorname{rank}\left(0,1_{\mu-r}\right) C_{1}=\mu-r$. Thus we have shown that representatives of $\Delta_{n, r} \backslash \Gamma_{n}$ are in $\left\{\sigma \uparrow\left({ }^{t} u{ }_{u^{-1}}\right) \mid \sigma \in \Gamma_{\mu}\right.$ with $\operatorname{rank}\left(0,1_{\mu-r}\right) \sigma_{3}^{(\mu)}=\mu-r, u \in$ $\left.G L_{n}(\mathbf{Z})\right\}$. The completeness is easy.

For $\sigma \in \Gamma_{\mu}$, it is easy to see

$$
(f \| \sigma \uparrow)(z)=j\left(\sigma, z^{*}\right)^{-k} f\left(\sigma\left(z^{*}\right)_{1}\right)\left(\frac{\operatorname{det} \Im \sigma\left(z^{*}\right)}{\operatorname{det} \Im \sigma\left(z^{*}\right)_{1}}\right)^{s}\left(\frac{\operatorname{det} y}{\operatorname{det} y^{*}}\right)^{s},
$$

where $z^{*}=z\left[\begin{array}{c}1_{\mu} \\ 0\end{array}\right]$ and the suffix 1 means $r \times r$ upper left submatrix. Thus $E_{n, r}^{k}(f, s ; q$, $M)$ turns out to be equal to

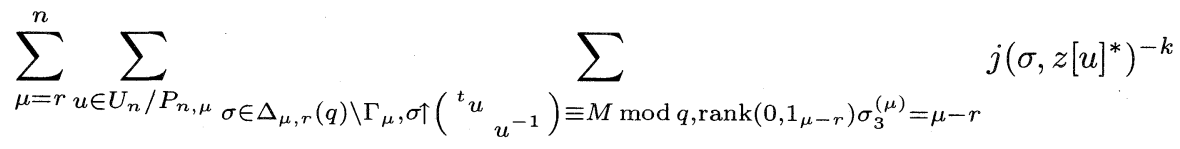

$$
\begin{aligned}
& \times f\left(\sigma\left(z[u]^{*}\right)_{1}\right)\left(\frac{\operatorname{det} \Im \sigma\left(z[u]^{*}\right)}{\operatorname{det} \Im \sigma\left(z[u]^{*}\right)_{1}}\right)^{s}\left(\frac{\operatorname{det} y}{\operatorname{det} y[u]^{*}}\right)^{s},
\end{aligned}
$$


where $w^{*}=w_{1}^{(\mu)}$. Hence for a regular half-integral matrix $T \in \Lambda_{n}^{\prime}$, the integration $\int_{x \in \Lambda_{n}(\mathbf{R}) / q \Lambda_{n}}(f \| \sigma \uparrow)(z) e(-\operatorname{tr} T x / q) d x$ vanishes for $\sigma \in \Gamma_{\mu}$ with $\mu<n$ and then by termwise integration Theorem 1 shows

$$
\int_{x \in \Lambda_{n}(\mathbf{R}) / q \Lambda_{n}} E_{n, r}^{k}(f, s ; q, M) e(-\operatorname{tr} T x / q) d x=q^{r(r+1) / 2} \mathcal{F}(T, y ; f, M) .
$$

REMARK 1. In Theorems 1, 2, $\Re s$ should be sufficiently large to justify the calculation. If $k>n+r+1$, then $\Re s \geq 0$ is enough and hence we can put $s=0$ in Theorems 1, 2.

Remark 2. To get $\Gamma_{0}$-type Klingen's Eisenstein series, we have only to add $E_{n, r}^{k}(f, s ; q, M)$ on $M \in N \Gamma_{0}$ for some $N \in \Gamma_{n}$. When we consider $\Gamma_{0}$-type, Euler factors turn out to be simpler for primes dividing level. When $N=1_{2 n}$, there is a result of Böcherer-Schulze-Pillot [BS].

REMARK 3. The author does not know when $E_{n, r}^{k}(f, 0 ; q, M)$ is a well-defined modular form $(k \leq n+r+1)$ and whether they span the space of modular forms of degree $n$, weight $k$, level $q$ with cusp forms and Eisenstein series or not. For the application to quadratic forms we need an estimate on $\mathcal{F}\left(T, T^{-1} ; f, M\right)$ at $s=0$ for $0<T \in \Lambda_{n}^{\prime}$.

\section{References}

[B] S. Böcherer Über die Fourier-Jacobi-Entwicklung Siegelscher Eisensteinreihen, Math.Z., 183 (1983), 21-46.

[BS] S. Böcherer, R. Schulze-Pillot, On a theorem of Waldspurger and on Eisenstein series of Klingen type, Math. Ann., 288 (1990), 361-388.

[D] S. Delsarte, Fonctions de Möbius sur les groupes abeliens finis, Ann. of Math., 49 (1948), $600-609$.

[DS] W. Duke and R. Schulze-Pillot, Representation of integers by positive ternary quadratic forms and equidistribution of lattice points on ellipsoids, Invent. Math., 99 (1990), 4957.

[K] H. Klingen, Introductory lectures on Siegel modular forms, Cambridge University Press, (1990).

[K1] Y. Kitaoka, Modular forms of degree $n$ and representation by quadratic forms, Nagoya Math. J., 74 (1979), 95-122.

[K2] Y. Kitaoka, Dirichlet series in the theory of Siegel modular forms, Nagoya Math. J., 95 (1984), 73-84.

[K3] Y. Kitaoka, Lectures on Siegel modular forms and representation by quadratic forms, Springer-Verlag, Heidelberg, (1986).

[K4] Y. Kitaoka, A note on Klingen's Eisenstein series, Abh. Math. Sem. Univ. Hamburg, 60 (1990), 95-114.

[R1] S. Raghavan, Estimates of coefficients of modular forms and generalized modular relations, "Automorphic forms, Representation theory and Arithmetic in Bombay," (1979).

[R2] S. Raghavan, Estimation of Fourier coefficients of Siegel modular forms, Math. Göttingensis, (1986). 
Department of Mathematics

SCHOOL OF SCIENCE

NAGOYA UNIVERSITY

Chikusa-KU, NAgOYA 464-01

JAPAN 